\title{
Damage-Mitigating Control of Space Propulsion Systems for High Performance and Extended Life
}

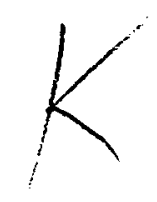

Asok Ray and Min-Kuang Wu

Pennsylvania State University

University Park, Pennsylvania

March 1994

Prepared for

Lewis Research Center

Under Grant NAG3-1240 


\title{
DAMAGE-MITIGATING CONTROL OF SPACE PROPULSION SYSTEMS FOR HIGH PERFORMANCE AND EXTENDED LIFE
}

\author{
Report Prepared for
}

NASA Lewis Research Center

under Grant\#: NAG3-1240

by

The Pennsylvania State University

University Park, PA 16802

\begin{abstract}
A major goal in the control of complex mechanical systems such as spacecraft rocket engines, advanced aircraft, and power plants is to achieve high performance with increased reliability, availability, component durability, and maintainability. The current practice of decision and control systems synthesis focuses on improving performance and diagnostic capabilities under constraints that often do not adequately represent the materials degradation. In view of the high performance requirements of the system and availability of improved materials, the lack of appropriate knowledge about the properties of these materials will lead to either less than achievable performance due to overly conservative design, or over-straining of the structure leading to unexpected failures and drastic reduction of the service life. The key idea in this report is that a significant improvement in service life could be achieved by a small reduction in the system dynamic performance. The major task is to characterize the damage generation process, and then utilize this information in a mathematical form to synthesize a control law that would meet the system requirements and simultaneously satisfy the constraints that are imposed by the material and structural properties of the critical components.

The concept of damage mitigation is introduced for control of mechanical systems to achieve high performance with a prolonged life span. A model of fatigue damage dynamics is formulated in the continuous-time setting, instead of a cycle-based representation, for direct application to control systems synthesis. An optimal control policy is then formulated via nonlinear programming under specified constraints of the damage rate and accumulated damage. The results of simulation experiments for the transient upthrust of a bipropellant rocket engine are presented to demonstrate efficacy of the damage-mitigating control concept.
\end{abstract}




\section{TABLES OF CONTENTS}

Page

ABSTRACT

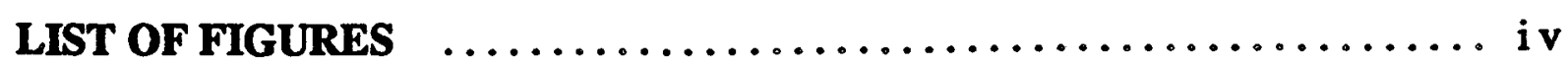

LIST OF TABLES $\ldots \ldots \ldots \ldots \ldots \ldots \ldots \ldots \ldots \ldots \ldots \ldots \ldots \ldots \ldots$ vi

CHAPTER 1. INTRODUCTION $\ldots \ldots \ldots \ldots \ldots \ldots \ldots \ldots \ldots \ldots \ldots \ldots$

1.1. Motivation and Statement of the Research Problem $\ldots \ldots \ldots \ldots \ldots 1$

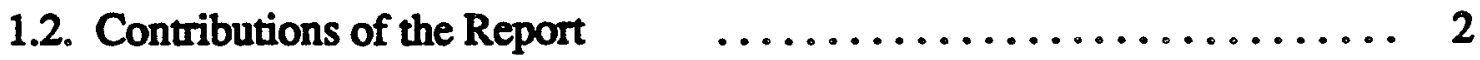

1.3. Organization of the Report $\quad \ldots \ldots \ldots \ldots \ldots \ldots \ldots \ldots \ldots, 2$

CHAPTER 2. DAMAGE-MITIGATING CONTROL SYSTEM $\ldots \ldots \ldots 4$

2.1. General Structure of the Damage-Mitigating Control System ....... 4

2.2. Formulation of the Damage-Mitigating Control System $\ldots \ldots \ldots \ldots 6$

\section{CHAPTER 3. FORMULATION OF AN OPTIMAL OPEN-LOOP} CONTROL POLICY $\ldots \ldots \ldots \ldots \ldots \ldots \ldots \ldots \ldots, 8$

3.1. Problem Formulation $\ldots \ldots \ldots \ldots \ldots \ldots \ldots \ldots \ldots \ldots, 8$

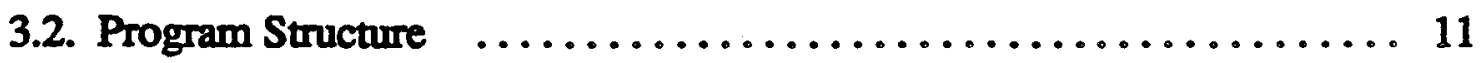

CHAPTER 4. MODELING OF FATIGUE DAMAGE DYNAMICS $\ldots \ldots \ldots 13$

4.1. A Review of Strain-Life Approach $\ldots \ldots \ldots \ldots \ldots \ldots \ldots \ldots \ldots$

4.2. Continuous-Time Damage Model ................... 17

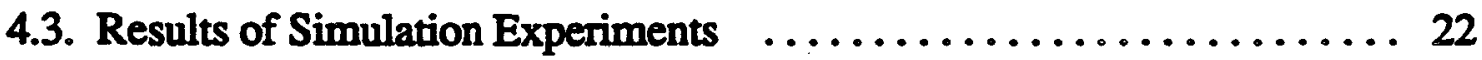

4.4. Modeling of Nonlinear Cumulative Damage Using Damage Curve

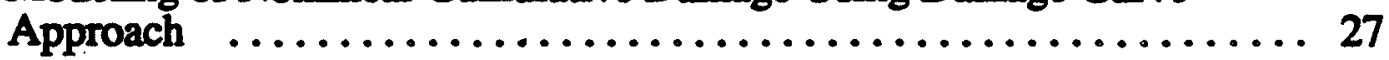

4.5. The $\gamma$-Parameter Fitting for the Nonlinear Cumulative Damage Model . 33

4.6. Continuous-Time Damage Modeling of Fatigue Crack Growth ..... 37

CHAPTER 5. SIMULATION RESULTS AND DISCUSSIONS $\ldots \ldots \ldots \ldots 39$

5.1. Simulation of the Bipropellant Rocket Engine under Open-Loop

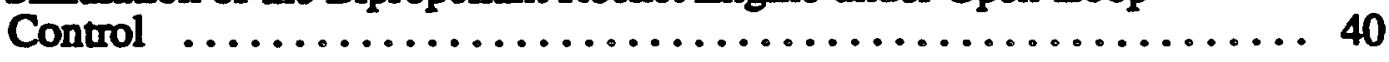

5.2. Results and Discussions $\ldots \ldots \ldots \ldots \ldots \ldots \ldots \ldots \ldots \ldots \ldots \ldots$ 
CHAPTER 6. SUMMARY, CONCLUSIONS AND RECOMMENDATIONS FOR FUTURE RESEARCH $\ldots \ldots \ldots \ldots \ldots \ldots \ldots \ldots . \ldots 7$

6.1. Recommendations for Future Research $\ldots \ldots \ldots \ldots \ldots \ldots \ldots \ldots .57$

APPENDIX A. CLOSED-LOOP CONTROL $\ldots \ldots \ldots \ldots \ldots \ldots \ldots \ldots$

APPENDIX B. RAINFLOW CYCLE COUNTING METHOD .........6 63

APPENDIX C. MODELING OF PLANT DYNAMICS $\ldots \ldots \ldots \ldots \ldots \ldots 65$

APPENDIX D. MODELING OF STRUCTURAL DYNAMICS $\ldots \ldots \ldots \ldots 75$

APPENDIX E. MODELING OF INTEGRATED FATIGUE-CORROSIONCREEP DAMAGE $\ldots \ldots \ldots \ldots \ldots \ldots \ldots \ldots \ldots \ldots, 82$

REFERENCES $\ldots \ldots \ldots \ldots \ldots \ldots \ldots \ldots \ldots \ldots \ldots \ldots \ldots \ldots \ldots \ldots \ldots \ldots$ 


\section{LIST OF FIGURES}

Page

Figure 2.1 General Structure of Damage-Mitigating Control System ....... 5

Figure 3.1 Nonlinear Programming Procedure $\ldots \ldots \ldots \ldots \ldots \ldots \ldots \ldots$

Figure 3.2 Program Structure for Open-Loop Control Policy via Nonlinear

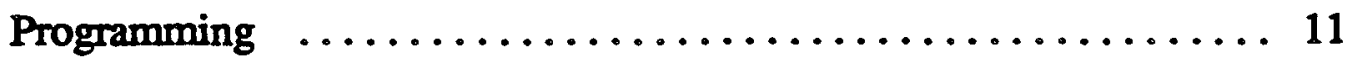

Figure 4.1 Strain-Life Curve Showing Elastic and Plastic Components $\ldots \ldots \ldots 14$

Figure 4.2 Cyclic Stress-Strain Curve $\ldots \ldots \ldots \ldots \ldots \ldots \ldots \ldots \ldots \ldots$

Figure 4.3 Definition of Damage Between Two Points Within a Reversal ..... 17

Figure 4.4 Integration When a Cycle Is Closed Between $t_{k}$ and $t_{k+1} \ldots \ldots \ldots 21$

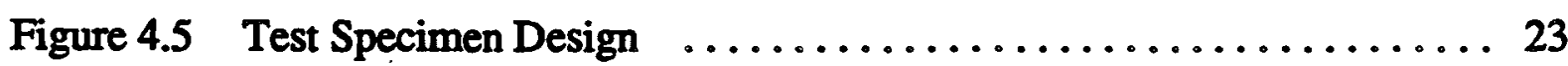

Figure 4.6 Comparison of Measured and Calculated Strain $\ldots \ldots \ldots \ldots . \ldots 23$

Figure 4.7 Comparison of Continuous-Time Damage Model and Strain-Life Approach for Maximum Load $=15.6 \mathrm{KN} \ldots \ldots \ldots \ldots \ldots \ldots 24$

Figure 4.8 Comparison of Continuous-Time Damage Model and Strain-Life Approach for Maximum Load $=35.6 \mathrm{KN} \ldots \ldots \ldots \ldots \ldots . \ldots 25$

Figure 4.9 Comparison of Continuous-Time Damage Model and Strain-Life Approach for Maximum Load $=71.2 \mathrm{KN} \ldots \ldots \ldots \ldots \ldots \ldots$

Figure 4.10 Nonlinear Damage Curve under Constant Amplitude Loading ..... 28

Figure 4.11 Nonlinear Damage Accumulation $\left(\Delta \sigma_{1}>\Delta \sigma_{2}\right) \ldots \ldots \ldots \ldots . \ldots 29$

Figure 4.12 Nonlinear Damage Accumulation $\left(\Delta \sigma_{1}<\Delta \sigma_{2}\right) \ldots \ldots \ldots \ldots . . .29$

Figure 4.13 Nonlinear Damage Increments $\ldots \ldots \ldots \ldots \ldots \ldots \ldots \ldots, \ldots \ldots$

Figure 4.14 Computation of Nonlinear Cumulative Damage $\ldots \ldots \ldots \ldots . \ldots 33$

Figure $4.15 \gamma_{\text {versus }} \mathrm{D}$ at Different Amplitudes $\ldots \ldots \ldots \ldots \ldots \ldots \ldots$

Figure 4.16 Nonlinear Damage Curves at Different Stress Amplitudes ...... 36 
Figure 5.1 Schematic Diagram of a Bipropellant Rocket Engine $\ldots \ldots \ldots \ldots 39$

Figure 5.2 Transient Responses of Preburner $\mathrm{O}_{2}$ Valve Areas $\ldots \ldots \ldots \ldots 44$

Figure 5.3 Transient Responses of Combustor $\mathrm{O}_{2}$ Valve Areas $\ldots \ldots \ldots \ldots 45$

Figure 5.4 Transient Responses of Preburner $\mathrm{O}_{2}$ Inlet Flow $\ldots \ldots \ldots \ldots \ldots 46$

Figure 5.5 Transient Responses of Combustor $\mathrm{O}_{2}$ Inlet Flow $\ldots \ldots \ldots \ldots \ldots 47$

Figure 5.6 Transient Responses of $\mathrm{H}_{2}$ Mass Flow $\ldots \ldots \ldots \ldots \ldots \ldots \ldots$

Figure 5.7 Transient Responses of $\mathrm{O}_{2} / \mathrm{H}_{2}$ Ratio $\ldots \ldots \ldots \ldots \ldots \ldots \ldots . \ldots 9$

Figure 5.8 Transient Responses of Preburner Pressure $\ldots \ldots \ldots \ldots \ldots \ldots$

Figure 5.9 Transient Responses of Combustor Pressure $\ldots \ldots \ldots \ldots \ldots \ldots$

Figure 5.10 Transient Responses of Preburner Temperature ............ 52

Figure 5.11 Transient Responses of Combustor Temperature $\ldots \ldots \ldots \ldots \ldots 53$

Figure 5.12 Transient Responses of Mean Stress $\ldots \ldots \ldots \ldots \ldots \ldots \ldots . \ldots$

Figure 5.13 Transient Responses of Damage Rate $\ldots \ldots \ldots \ldots \ldots \ldots \ldots 5$

Figure 5.14 Transient Responses of Accumulated Damage $\ldots \ldots \ldots \ldots \ldots$

Figure A.1 Closed-Loop Damage Control System $\ldots \ldots \ldots \ldots \ldots \ldots \ldots 6$

Figure B.1 Rainflow Cycle Counting Method $\ldots \ldots \ldots \ldots \ldots \ldots \ldots$

Figure C.1 Model Solution Diagram of a Bipropellant Rocket Engine $\ldots \ldots 65$

Figure D.1 Flow Chart for the Structural Model of a Turbine Blade ........ 75

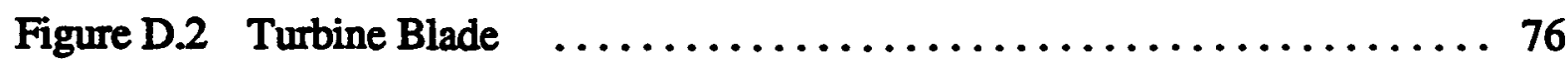

Figure D.2 Finite Element Model of a Turbine Blade $\ldots \ldots \ldots \ldots \ldots \ldots \ldots 77$ 


\section{LIST OF TABLES}

Page

Table 4.1 Smooth Specimen Stress-Strain and Fatigue Properties $\ldots \ldots \ldots \ldots 22$

Table 4.2 Effective Stress Intensity Factor Range versus Crack Growth Rate Relationship $\ldots \ldots \ldots \ldots \ldots \ldots \ldots \ldots \ldots \ldots \ldots \ldots . \ldots . \ldots$

Table 4.3 Material Properties of AISI $4340 \quad \ldots \ldots \ldots \ldots \ldots \ldots \ldots \ldots$

Table 5.1 The Constraints for the Two Cases under Simulation Condition 1 .... 41

Table C.1 Plant Control Input, Measured Output and State Variables $\ldots \ldots \ldots 72$

Table C.2 System Matrices for the Linearized Plant Model $\ldots \ldots \ldots \ldots \ldots 73$ 


\section{CHAPTER 1}

\section{INTRODUCTION}

\subsection{Motivation and Statement of the Research Problem}

A major goal in the control of complex mechanical systems such as spacecraft rocket engines, advanced aircraft, and power plants is to achieve high performance with increased reliability, availability, component durability, and maintainability [Lorenzo and Merrill (1991a); Ray et al. (1993a, 1993b)] which manifest the following requirements:

- Extension of the service life of the controlled process;

- Increase of the mean time between major maintenance actions;

- Reduction of risk in the integrated control-structure-materials system design.

Therefore, the control systems need to be synthesized by taking performance, mission objectives, service life, and maintenance and operational costs into consideration. The current practice of decision and control systems synthesis focuses on improving dynamic performance and diagnostic capabilities under constraints that often do not adequately represent the materials degradation. The reason is that the traditional design is based upon the assumption of conventional materials with invariant characteristics. In view of high performance requirements and availability of improved materials that may have significantly different damage characteristics relative to conventional materials, the lack of appropriate knowledge about the properties of these materials will lead to either of the following:

- Less than achievable performance due to overly conservative design; or

- Over-straining of the structure leading to unexpected failures and drastic reduction of the useful life span.

For example, reusable rocket engines present a significantly different problem in contrast to expendable propulsion systems that are designed on the basis of minimization of weight and acquisition cost under the constraint of specified system reliability. In the reusable rocket engines, multiple start-stop cycles cause large thermal strains; steady-state stresses generate inelastic strains; and dynamic loads induce high cyclic strains leading to fatigue failures. The original design goal of the Space Shuttle Main Engine (SSME) was specified for 55 flights before any major maintenance, but the current practice is to disassemble the engine after each flight for maintenance [Lorenzo and Merrill (1991a)]. Another example is design modification of the F-18 aircraft as a result of conversion of the flight control system from analog to digital, which would lead to a significant change in the load spectrum on the airframe structure. In this case, a major goal of the vehicle control systems redesign should be to achieve a trade-off between flight maneuverability, and durability of the critical components [Noll et al. (1991)].

As the science and technology of materials continue to evolve, the design methodologies for damage-mitigating control must have the capability of easily 
augmentation of current system-theoretic and AI-based techniques for synthesis of decision and control laws with governing equations and inequality constraints that would model the mechanical properties of the materials for the purpose of damage representation and failure prognosis. The major challenge in this research is to characterize the damage generation process, and then utilize this information in a mathematical form for synthesizing the control algorithms in complex mechanical systems.

Although a significant amount of research has been conducted in each of the individual areas of Control Engineering and Analysis \& Prediction of Materials Damage, integration of these two disciplines has not received much attention. Recently, Noll et al. (1991) have pointed out the need for interdisciplinary research in the fields of active control technology and structural integrity, specifically fatigue life analysis, in view of the integrated structural and flight control of advanced aircraft. Lorenzo and Merrill (1991b) have proposed a concept of damage mitigation and failure prognosis in the framework of reusable rocket engines for space propulsion in the context of intelligent control and diagnostics. This intelligent control system is hierarchically structured in which the top level coordinates the major functionalities of life extending control, adaptive control, real-time diagnostics and prognostics, and sensor/actuator fault accommodation. A major goal is to ensure safety and achieve the mission objectives while arbitrating the potentially conflicting requirements of optimum performance and structural durability. However, this concept of damage mitigation is not restricted to intelligent control and diagnostics, and can be applied to any system where structural durability is an important issue.

\subsection{Contributions of the Report}

The research reported in this report addresses the two different disciplines of Control Systems Engineering and Fracture Mechanics. The major contribution of this research is the development of a usable damage model and its application to the damage mitigating control of complex mechanical structures. In contrast to the usual notion of expressing the fatigue damage rate relative to the number of cycles, a concept of time derivative of the damage has been developed based on the conventional life prediction methods. A unique advantage of this damage model in the continuous-time setting is that it can be directly incorporated within the control system structure to provide the necessary information for on-line damage-mitigating control as well as for off-line synthesis of the control system.

The above concept of continuous-time damage modeling can be related to a variety of cycle-based life prediction methods. This is essential for synthesis of a damage-mitigating control system since engineering applications may adopt different methodologies for damage analysis due to the diversity of engineering materials and damage accumulation mechanisms.

\subsection{Organization of the Report}

The report is organized in six chapters, including the introduction, and five appendices. Chapter 2 presents a general structure of the damage-mitigating control system along with a description of each component. Chapter 3 describes the procedure of synthesizing an open-loop control law via nonlinear programming to optimize the 
system performance without violating the imposed constraints. Chapter 4 reviews the current life prediction methods and derives the continuous-time damage model based on an experimentally verified approach. The control system is then simulated on the basis of a simplified model of the space shuttle main engine (SSME), and the results of simulation experiments are discussed in Chapter 5. Chapter 6 summarizes the potential problems and difficulties in the current approach and suggests possible solutions and the areas of future research. Five appendices provide the necessary information to complement the results reported in the main body of this report. Appendix A depicts the structure of a closed-loop control system with discussions on robustness and reliability. Appendix B explains the basic principle of a cycle counting approach for fatigue life prediction under spectral loading. Appendix $C$ presents a mathematical model of a bipropellant rocket engine which is the plant under control. Appendix D describes the stress analysis of a turbine blade which is considered to be a critical component of the plant. Finally, an approach for modeling of integrated fatiguecorrosion-creep damage is outlined in Appendix E. 


\section{CHAPTER 2}

\section{DAMAGE-MITIGATING CONTROL SYSTEM}

The damage-mitigating control system (DCS), also referred to as Life Extending Control system [Lorenzo and Merrill (1991b)], is intended to function independently or as an integral part of a hierarchically structured control system. The DCS may be centralized or distributed depending on the spatial location of the critical plant components and the overall objective of the mission. As stated in Chapter 1, the major challenge in the DCS design is to characterize the damage generation process such that this information can be directly applied to synthesize the control law. Therefore, this report focuses on the fundamental issue of formulating a generic structure of the DCS with the objective of achieving high performance while simultaneously maintaining the accumulated damage and the damage rate of the critical plant component(s) within the prescribed limits.

\subsection{General Structure of the Damage-Mitigating Control System}

Figure 2.1 shows a conceptual view of the $D C S$ which consists of the following: (i) a physical plant under control; (ii) a damage predictor which models the structural and damage dynamics of the critical plant components; and (iii) a feedback controller which coordinates the plant states and damage mitigation. Referring to the control systems structure in Figure 2.1, the DCS synthesis consists of the following major tasks:

- Task 1: Formulation of the plant model to represent the dynamic characteristics of the physical process;

- Task 2: Formulation of the damage predictor to represent the dynamic characteristics of the structural and material properties of the critical plant components ;

- Task 3: Synthesis of an open-loop control policy via optimization of a cost functional (representing the mission objectives) under the constraints imposed by the plant model and the damage prediction model; and

- Task 4: Synthesis of a closed-loop control policy via feedback of the plant states and damage information to steer the plant along the open-loop trajectory without exceeding the prescribed damage constraints.

As shown in Figure 2.1, the sequence of open-loop control commands, $\left\{\mathbf{u f f}^{\mathrm{ff}}\right.$, serve as the feedforward input to the plant to fulfill the mission objectives under the specified damage constraints. The plant model is a finite-dimensional state-space representation of the system dynamics (e.g., thermal-hydraulic dynamics of the space shuttle main engine or propulsion and aerodynamics of an aircraft). The estimated plant states and plant outputs are the inputs to the structural model which, in turn, generates the necessary information for the damage model. The output of the structural model is the load vector which may consist of (time-dependent) stress, strain, temperature, wear, level of corrosion in gaseous and aqueous environments, and other physico-chemical process variables at the critical point(s) of the structure. The damage model is a continuous-time as opposed to a cycle-based representation of life prediction so that it can be integrated with the plant and structural models for DCS synthesis. This damage model includes the effects of damage rate and accumulated damage at the critical point(s) of the structure that are subjected to the time-dependent load. The damage 
state vector $\mathbf{v}(t)$ could indicate the level of micro-cracking, macroscopic crack length, wear, creep, corrosion, density of slip bands, etc., at one or more critical points, and its time derivative $\dot{v}(t)$ indicates how the instantaneous load is affecting the structural

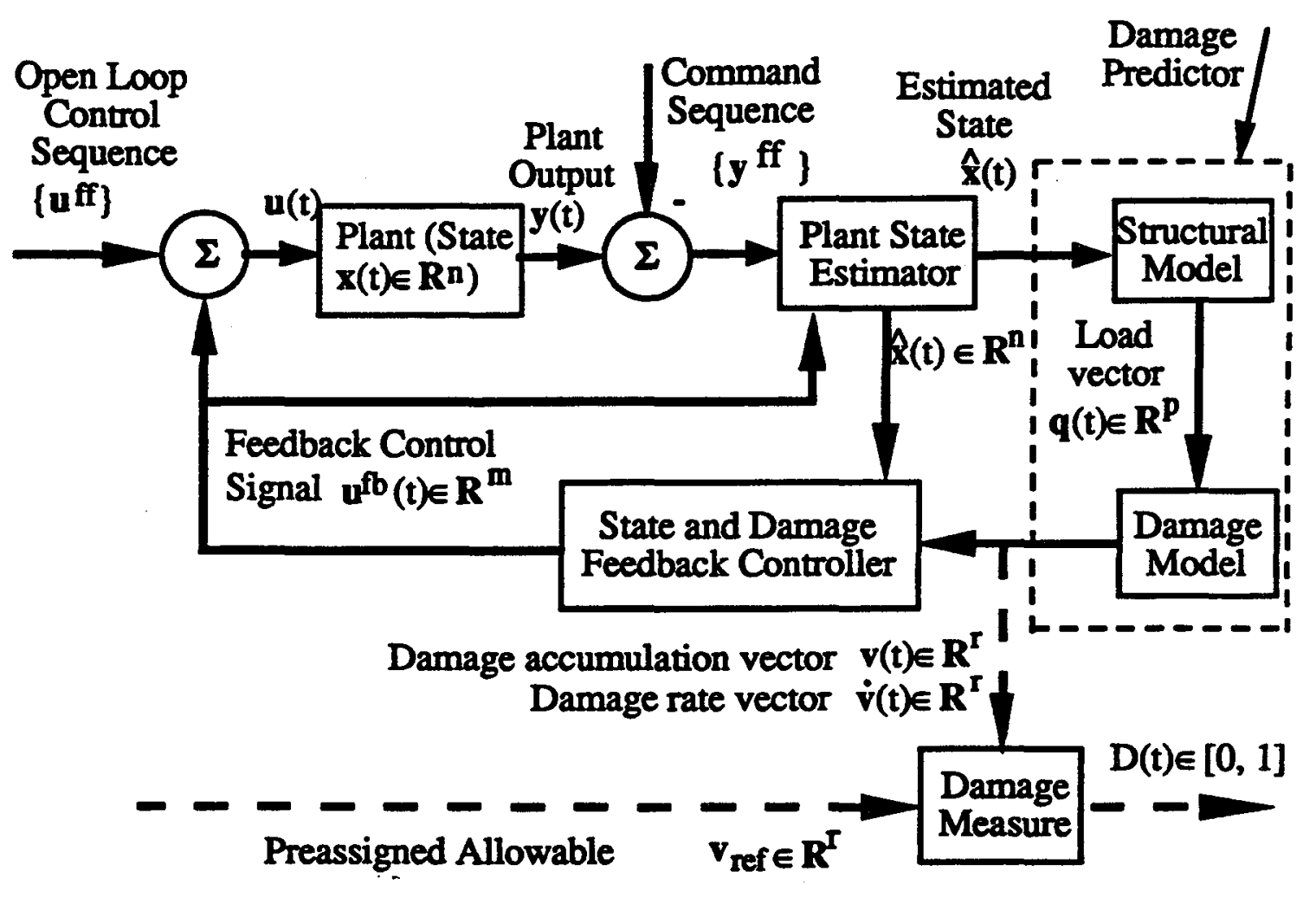

Figure 2.1 General Structure of Damage-Mitigating Control System

components. These two vectors, $\mathbf{v}(t)$ and $\dot{v}(t)$, are the damage variables to be constrained in the synthesis of an optimal open-loop control policy, and also provide the important information for damage mitigation in the synthesis of the feedback control policy. The overall damage $\mathrm{D}(\mathrm{t})$ is a scalar measure of the combined damage at one or more critical points resulting from different effects (e.g., fatigue, creep, corrosion, or wear) relative to the preassigned allowable level $\mathbf{v}_{\text {ref }}$ of the damage vector. Although $D(t)$ may not directly enter the feedforward or feedback control loop, it can provide useful information for intelligent decision-making such as damage prognosis and risk analysis. In the closed-loop control, the state and damage feedback controller monitors and controls the plant and the damage states which may deviate from the desired values due to modeling uncertainties, external disturbances, and sensor noise. The role of this controller is to manage the possible conflicts between the plant performance and damage, and generate the appropriate feedback control efforts, $\mathbf{u}^{\mathrm{fb}}$, to maintain the plant response as close as possible to the nominal trajectory of the openloop control policy. 


\subsection{Formulation of the Damage-Mitigating Control System}

The dynamics of each block in Figure 2.1 need to be modeled in an appropriate form so that it can be directly applied to the synthesis of both the open-loop and closedloop control laws. The plant dynamics and damage predictor are modeled by nonlinear (and possibly time-varying) differential equations which must satisfy the local Lipschitz condition [Vidyasagar (1992)] within the domain of the plant operating range. The structural model consists of solutions of dynamic equations representing the structural properties and the (mechanical and thermal) load conditions. These equations may be generated using a variety of analytical techniques ranging from finite element analysis to simple relationships of uniaxial stress and strain. However, the transformations relating the structural responses (such as nodal displacements) to the stress-strain behavior need to be included in the model. Since control systems are analyzed in the continuous-time setting, it would be necessary to express the damage equations in terms of time as the independent variable instead of the number of cycles. The continuoustime damage model is a necessity for practical implementation in the control synthesis procedure and, most importantly, generation of the intra-cycle damage information. The general formulation of the plant and damage dynamics and their constraints are represented in the deterministic setting as follows:

Task period: Starting time to $_{0}$ to final time $\mathbf{t}_{\mathbf{f}}$

Plant dynamics : $\quad \frac{d x}{d t}=f(x(t), u(t), t) ; \quad x\left(t_{0}\right)=x_{0}$

Damage dynamics : $\quad \frac{d v}{d t}=h(v(t), q(x, t), t) ; \quad v\left(t_{0}\right)=v_{0} ; \quad h \geq 0 ; \quad \forall t$

Damage measure : $\quad D(t)=\xi\left(v(t), v_{\text {ref }}\right)$ and $D(t) \in[0,1]$

Damage rate tolerance : $\quad 0 \leq h(v(t), q(x, t), t)<\beta(t) ; \quad \forall t \in\left[t_{0}, t_{t}\right] ;$

Accumulated damage tolerance : $\left[v\left(t_{f}\right)-v\left(t_{0}\right)\right]<\varphi$ where

$\mathbf{x} \in \mathbf{R}^{\mathfrak{n}}$ is the plant state vector;

$\mathbf{u} \in \mathbf{R}^{\mathbf{m}}$ is the control input vector;

$v \in R^{r}$ is the damage state vector,

$\mathbf{v}_{\mathrm{ref}} \in \mathbf{R}^{\mathbf{r}}$ is the preassigned limit for the damage state vector,

$\beta(t) \in R^{r}$ and $\varphi \in R^{r}$ are specified tolerances for the damage rate and accumulated damage, respectively;

$\mathbf{y} \in \mathbf{R}^{\mathbf{P}}$ is the plant output vector;

$\hat{\mathbf{x}} \in \mathbf{R}^{\mathbf{n}}$ is an estimate of the plant state vector,

$q \in \mathbf{R}^{\mathbf{p}}$ is the load vector, and

$D \in[0,1]$ is a scalar measure of the accumulated damage. 
The vector differential equations (2.1) and (2.2) become stochastic if the randomness of plant and material parameters is included in the models, or if the plant is excited by discrete events occurring at random instants of time [Sobczyk and Spencer (1992)]. The stochastic aspect of damage-mitigating control is a subject of future research.

The proposed DCS in Figure 2.1 uses the concept of conventional state estimation and state feedback together with the information of damage rate and accumulated damage to generate an appropriate feedback control signal. Although this additional information renders the control system to be highly nonlinear, the dynamic performance and service life of the plant can be better managed with damage mitigation. Without the damage feedback, the controller depicted in Figure 2.1 would reduce to a conventional output feedback controller. This report focuses on the development of a continuous-time damage model and formulation of an open loop control policy. Although the synthesis of a closed loop control system is not within the scope of this report, this topic is briefly discussed in Appendix A. 


\section{CHAPTER 3}

\section{FORMULATION OF AN OPTIMAL OPEN-LOOP CONTROL POLICY}

Given an initial condition, an open-loop policy for the plant control is generated via nonlinear programming [Luenburger (1984)] by optimizing the plant dynamic performance without violating the prescribed upper bounds of the damage rate and the accumulated damage as discussed in Chapter 2. The open-loop control synthesis procedure is developed in this chapter.

\subsection{Problem Formulation}

The problem is to identify an open-loop control policy by minimizing a cost functional under the constraints of the damage rate and the accumulated damage over a period of time. The cost functional, $\mathrm{J}$, is chosen to be the square of the weighted $\mathrm{L}_{2}$ norm of the plant states, damage rate, and control inputs. During the task period, [ to, $t_{f}$, the plant is steered from its initial state $x\left(t_{0}\right)$ to the final state $\mathbf{x}\left(t_{f}\right)$ along an optimal trajectory. In the formulation of an optimization problem, the control functions, $u(t), t$ $\in\left[t_{0}, t_{f}\right]$, needs to be discretized in time as a sequence $\left\{u_{k}\right\}$ so that the number of variables to be optimized is finite for implementation on a finite-state machine. Partitioning the task period [ $\left.\mathbf{t}_{0}, t_{\mathrm{f}}\right]$ into $\mathbf{N}$ steps at the discrete time instants $\left\{\mathrm{t}_{\mathrm{k}}\right\}$, $k=0,1,2, \cdots \infty, N$, integration of the plant and damage dynamics in eqs. (2.1) and (2.2) of Chapter 2 yields the following results:

$$
\text { Discretized plant dynamics : } \quad \mathbf{x}_{\mathbf{k}+1}=\mathbf{x}_{\mathbf{k}}+\int_{\mathbf{t}_{\mathbf{k}}}^{t_{\mathbf{k}+1}} \mathbf{f}(\mathbf{x}(\mathrm{t}), \mathbf{u}(\mathrm{t}), \mathrm{t}) \mathrm{dt} ;
$$

$$
\text { Discretized damage dynamics : } \mathbf{v}_{\mathbf{k}+1}=\mathbf{v}_{\mathbf{k}}+\int_{\mathbf{t}_{\mathbf{k}}}^{t_{\mathbf{k}+1}} \mathbf{h}(\mathbf{v}(\mathrm{t}), \mathbf{q}(\mathrm{x}, \mathrm{t}), \mathrm{t}) \mathrm{dt} \text {; }
$$

where the plant state, damage state and damage rate vectors are denoted as:

$$
x_{k}=x\left(t_{k}\right) ; v_{k}=v\left(t_{k}\right) ; \text { and } \dot{v}_{k}=h\left(v_{k}, q\left(x_{k}, t_{k}\right), t_{k}\right)
$$

Then, the optimization problem is formulated in a general form as follows:

$$
\text { Minimize: } J=\sum_{k=0}^{N-1} J_{k}\left(\tilde{\mathbf{x}}_{k}, \dot{\mathbf{v}}_{\mathbf{k}}, \tilde{\mathbf{u}}_{\mathbf{k}}\right)
$$

Subject to : $0 \leq h\left(v_{k}, q\left(x_{k}, t_{k}\right), t_{k}\right)<\beta(k)$ for $k=1,2,3, \cdots, N$; and

$$
\left(v_{N}-v_{0}\right)<\varphi
$$

where $\tilde{\mathbf{x}}_{k}=\mathbf{x}_{k}-\mathbf{x}_{s s}$ and $\tilde{u}_{k}=u_{k}-u_{s s}$ are deviations of the plant state vector and the control input vector from the respective final steady state values of $\mathbf{x}_{\mathbf{s s}}$ and $\mathbf{u}_{\mathrm{ss}}$; and $\beta(\mathrm{k})$ $\in \mathbf{R}^{\mathbf{T}}$ and $\phi \in \mathbf{R}^{\mathbf{r}}$ are specified tolerances for the damage rate and accumulated damage, respectively. 
The open-loop control law is synthesized by minimizing the cost functional in eq. (3.3) under: (i) the above inequality constraints in eq. (3.4); and (ii) the condition that, starting from the initial conditions $x\left(t_{0}\right)$ and $v\left(t_{0}\right)$, the state trajectory must satisfy the plant dynamic model in eq. (2.1). The design variables to be optimized are the discrete control inputs $u_{k}, k=0,1,2, \cdots, N-1$, and $u_{N}$ is not required to be identified because the optimization procedure is terminated at $t_{N}=t_{f}$. The flow chart shown in Figure 3.1 depicts a procedure for synthesizing an open-loop control policy via nonlinear programming.

The first step in the nonlinear programming is to formulate a mathematical model of the plant dynamics in the state space form of eq. (2.1) with proper initial conditions. Based on the responses of the plant at the normal operating condition, the critical component(s) in which the damage is most likely to occur is (are) identified either by stress analysis or from the history of plant operations. Upon identification of the critical points for damage prediction, the structural modeling and the continuoustime damage modeling are used to formulate the damage dynamics in the form of eq. (2.2). The resulting damage rate and accumulated damage are to be constrained in the optimization procedure via nonlinear programming. The upper bounds of the constraints on damage rate and accumulated damage need to be appropriately selected by taking the mission objectives, the time interval between maintenance, service life and allowable risk into consideration. The initial damage is important due to its significant effects on the dynamics of nonlinear damage accumulation. The performance cost functional in eq. (3.3), which is minimized under nonlinear programming, is a function of the plant states, and damage rate vectors, representing a trade-off between system performance and damage. The weights are assigned to the plant states or selected output variables reflecting their relative impact on the system performance. If the damage constraints are appropriately chosen, it may not be necessary to include the damage rate in the cost functional. Upon selection of the cost functional and damage constraints, the task is to find an optimal control sequence $\left[u_{k}\right.$ \} in discrete steps from time to to $t_{f}$. This optimal control sequence is then tested via system simulation and structural analysis to verify the plant performance and damage. If the results are satisfactory, the synthesis of an optimal policy for open-loop control is completed. Otherwise, the damage constraints should be revised and the optimization procedure is repeated to find a new control sequence $\left\{u_{k}\right\}$. It is also possible that an optimal solution may not exist due to overly stringent damage constraints. In that case, modifications of the constraints $\beta$ or $\varphi$, or of the cost functional J are needed. If none of these revisions are advisable, revision of the damage model or the plant model should be considered. For example, in some situations, an alternative approach such as the fatigue crack growth model may be more suitable than the cyclic strain approach for damage modeling (see Section 4.6).

In this report, a general purpose nonlinear programming package, namely the IMSL subroutine DNCONF [IMSL manual], has been adopted to solve the nonlinear optimization problem. Other nonlinear programming packages such as NPSOL [Gill et al. (1991)] of Stanford University are being considered for improving the computational efficiency in the future research. Following the optimization procedure in Figure 3.1, simulation experiments were carried out to evaluate the efficacy of the proposed damage-mitigating control. The results of simulation experiments are presented and discussed in Chapter 5. 


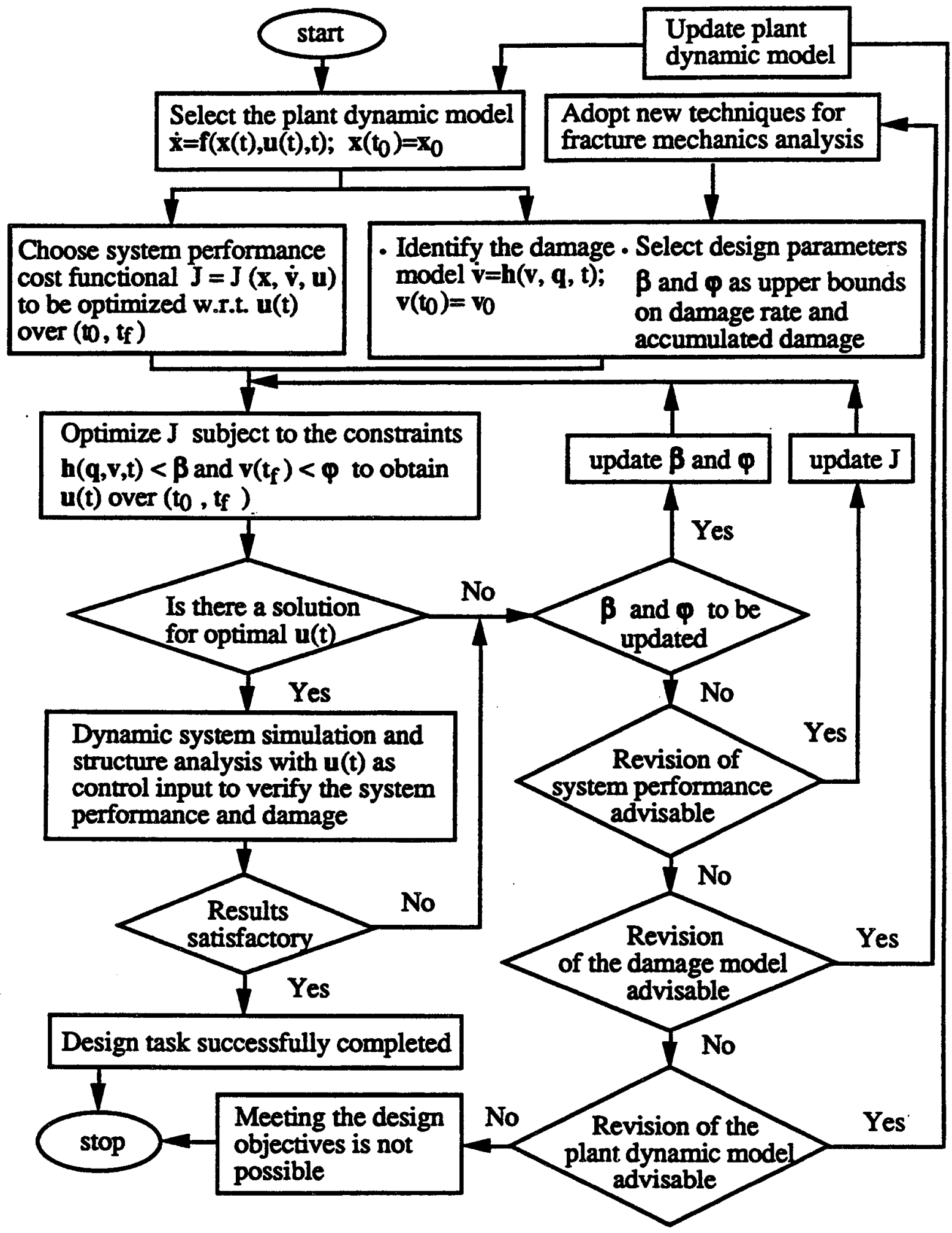

Figure 3.1 Nonlinear Programming Procedure 


\subsection{Program Structure}

The software for open-loop control synthesis in simulation experiments is coded in Fortran using the IMSL subroutine DNCONF, and the program structure is shown in Figure 3.2. The namelist data file on the left-hand top corner of Figure 3.2 contains the values of (i) the parameters of the plant model and the structural model, and (ii) material properties and damage constraints of the fatigue damage model. It serves as a data bank for the main program and supplies the necessary data to the subroutines through the COMMON statements. The MAIN program formats the output of the nonlinear programming (i.e., the optimal solutions: $\mathbf{x f f}^{\mathrm{ff}} \mathbf{u} \mathrm{ff}$, and $\dot{\mathbf{v} f}$ ), and these formatted data are to be used in the closed-loop control system in Figure 2.1 as the reference inputs.

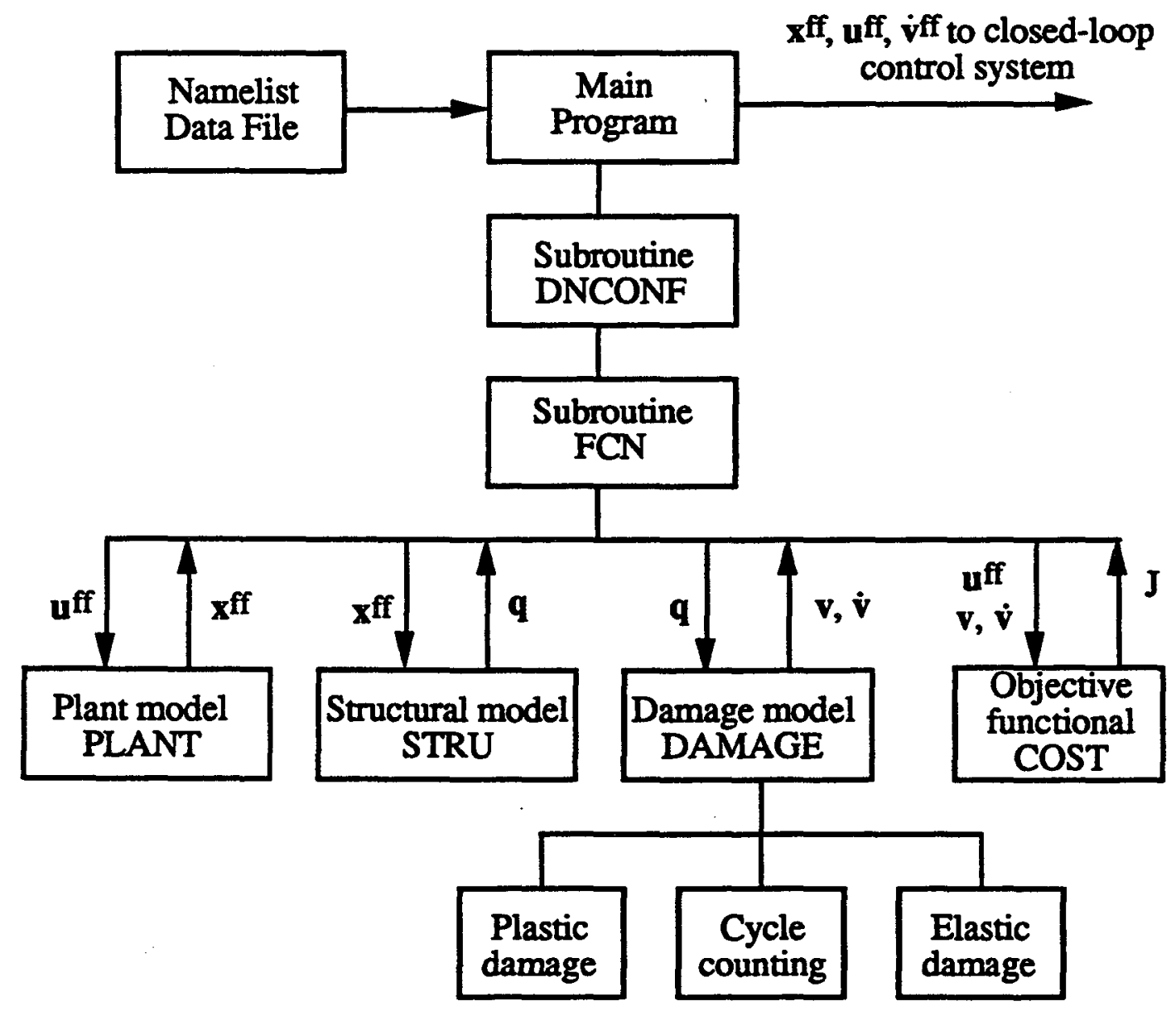

Figure 3.2 Program Structure for Open-Loop Control Policy via Nonlinear Programming

The IMSL subroutine DNCONF, called by the MAIN program, uses a line search algorithm that minimizes the cost functional J. An user-supplied external subroutine FCN is called by DNCONF to calculate the objective functional and the values of constraints. The variables to be evaluated are the (open-loop) control input

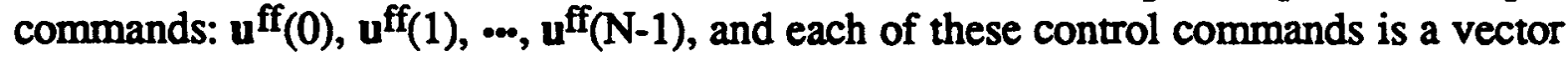


of dimension $\mathrm{m}$. Therefore, the total number of the variables to be optimized is equal to $\mathrm{m} \times \mathbf{N}$. At each step of the line search procedure, the sequence $\left\{u_{\mathrm{ff}}\right\}$ is updated representing the improvement of the cost functional. The subroutine PLANT consisting of the plant dynamic model equations is called by FCN to calculate the state vector $\mathbf{x}^{\mathrm{ff}}$ for each input sequence, and the results are available to the structural model for evaluation of the load vector, $\mathbf{q}$, which is the input to the damage model for prediction of the accumulated damage and damage rate. The damage rate, $\dot{\mathbf{v}}$, along with the state vector, $\mathbf{x}^{\mathrm{ff}}$, and control input, $\mathbf{u}^{\mathrm{ff}}$, are used by the subroutine COST to calculate the cost functional which, in turn, is fed back to the subroutine FCN for evaluation of the damage constraints. The line search continues until the cost functional converges and an optimal solution is reached. 


\section{CHAPTER 4}

\section{MODELING OF FATIGUE DAMAGE DYNAMICS}

Damage of mechanical structures is usually a result of fatigue, creep, corrosion, and their combinations [Suresh (1991)]. While the fatigue damage is cycle-dependent, the creep damage and corrosion damage are time-dependent. The prime focus in this report is on the representation of fatigue damage in a continuous-time setting. As discussed earlier, a time-dependent model of damage dynamics, having the structure of eq. (2.2), is necessary for analysis and synthesis of DCS. From this perspective, a dynamic model of fatigue damage has been formulated in the continuous-time setting. Although this damage model has a deterministic structure, it can be recast in the stochastic setting to include the effects of both unmodeled dynamics and parametric uncertainties. Augmentation of the fatigue damage model to include dynamics of corrosion and creep damage is briefly discussed in Appendix E.

Because of the wide ranges in mechanical properties of materials, extensive varieties of experiments have been conducted for fatigue analysis, and many models have been proposed for fatigue life prediction in aerospace and ground vehicles [Bannantine et al. (1990); Dowling (1983)]. Each of these models expresses the damage dynamics by an equation with the number of cycles as the independent variable. In contrast, the damage dynamics in eq. (2.2) are expressed as a vector differential equation with respect to time, $t$, as the independent variable. The advantages of this representation are that it allows the damage model to be incorporated within the time-based structure of the constrained optimization problem and that the damage accumulated between any two instants of time can be derived even if the stressstrain hysteresis loop is not closed. The damage information at any desired point within a cycle is computed following the proposed approach. This concept is applicable to different models of damage dynamics such as those based on local strain or crack propagation. The strain-life and linear elastic fracture mechanics approaches for fatigue life prediction are briefly described below.

- Cyclic Strain-Life: In this approach, the local stress-strain behavior is analyzed at certain critical points where failure is likely to occur. The local strain is directly measured from a strain gauge, or analytically computed. The local stress is estimated from the cyclic stress-strain curve. A cycle-based approach is then used to estimate the fatigue damage from the strain-life curves which are obtained through experiments at different levels of stress and strain amplitudes. The total accumulated damage is computed using the Palmgren-Miner rule [Miner (1945)] and subsequently modified via the damage curve approach [Bolotin (1989)].

- Linear Elastic Fracture Mechanics (LEFM): The LEFM approach is built upon the concept of a physical measure of damage in terms of the crack length and the size of the plastic zone at the crack tip. The accumulated damage is computed by integrating the crack growth rate over the number of cycles. This is based on the crack growth rate equation being approximated by an exponential function of stress intensity factor range of the component [Bannantine et al. (1990)]. The component is assumed to fail when the crack reaches the critical length which, in turn, is determined from the fracture toughness of the component on the basis of experimental data. 
Either of the above two approaches can be adopted for developing a continuous-time damage model depending on the availability of material data and the applications. The primary approach adopted in this report is based on cyclic strain-life. The next section reviews the governing equations and a procedure for fatigue damage prediction via the cyclic strain-life approach. An alternative approach based on LEFM is also presented in this chapter to demonstrate the applicability of the derivative concept.

\subsection{A Review of Strain-Life Approach}

The fatigue damage is primarily controlled by the local strain at the critical point(s) of the component, and is estimated by the strain-life curve which is essentially a plot of strain amplitudes $(\Delta \varepsilon / 2)$ versus the number of cycles to failure $\left(N_{f}\right)$ as obtained from constant amplitude fatigue tests on axially loaded specimens. A typical strain-life curve is shown in Figure 4.1. The relationship between $\Delta \varepsilon / 2$ and $N_{f}$ can be expressed in the following form:

where

$$
\frac{\Delta \varepsilon}{2}=\frac{\sigma_{f^{\prime}}}{E}\left(2 N_{f}\right)^{b}+\varepsilon_{f^{\prime}}\left(2 N_{f}\right)^{c}
$$

$\sigma_{\mathrm{f}}^{\prime}:$ fatigue-strength coefficient

$b$ : fatigue-strength exponent

$\varepsilon_{f^{\prime}}$ : fatigue-ductility coefficient

c : fatigue-ductility exponent

E : elastic modulus

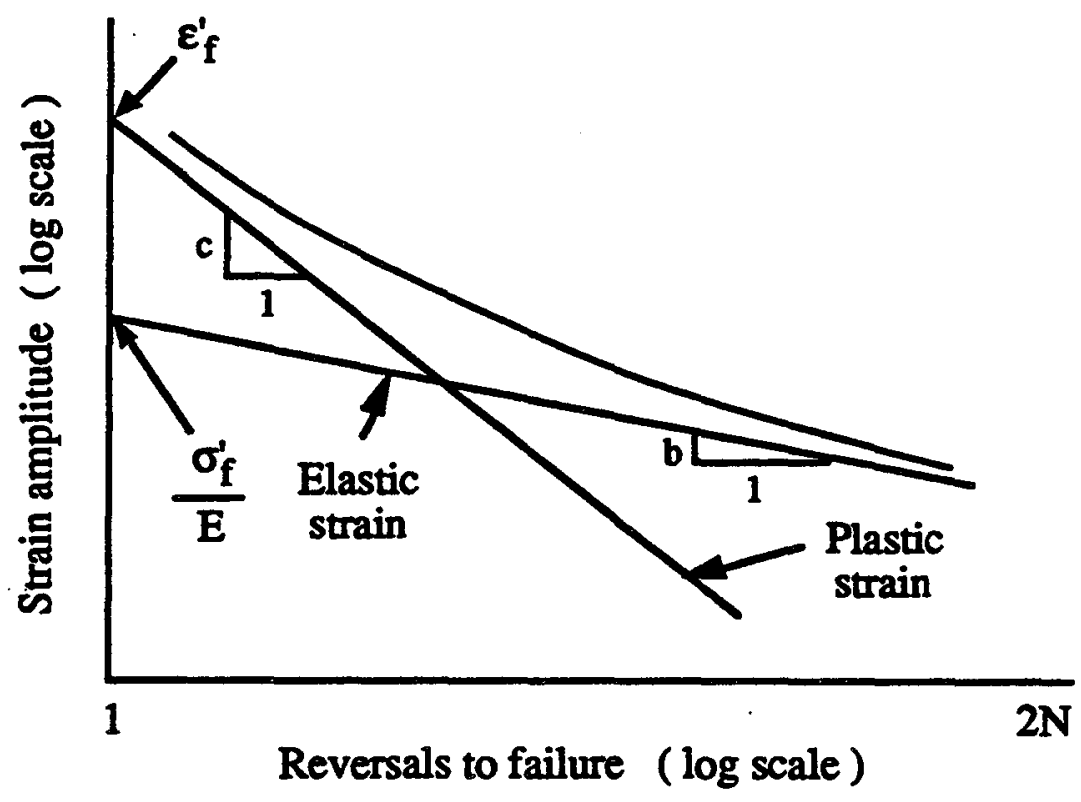

Figure 4.1 Strain-Life Curve Showing Elastic and Plastic Components 
The above coefficients are considered to be material constants which are available in the open literature [Tucker (1974); Boller and Seeger (1987)] for steel and aluminum alloys. The constant amplitude loading experiments are normally conducted under completely reversed strain control with zero mean stress. However, different mean stress levels have been found to have important effects on the fatigue life. One method [Fuchs and Stephens (1980)] is to replace $\sigma_{f}^{\prime}$ by $\sigma_{f}^{\prime}-\sigma_{m}$ in eq. (4.1) to account for the mean stress effect such that

$$
\frac{\Delta \varepsilon}{2}=\frac{\sigma_{f}^{\prime}-\sigma_{m}}{E}\left(2 N_{f}\right)^{b}+\varepsilon_{f}^{\prime}\left(2 N_{f}\right)^{c}
$$

where $\sigma_{m}$ is the mean stress. Dowling (1983) further modified equation (4.2) as:

$$
\frac{\Delta \varepsilon}{2}=\frac{\sigma_{f^{\prime}}-\sigma_{m}}{E}\left(2 N_{f}\right)^{b}+\varepsilon_{f^{\prime}}\left(1-\frac{\sigma_{m}}{\sigma_{f^{\prime}}}\right)^{c / b}\left(2 N_{f}\right)^{c}
$$

The total number of cycles to failure $\left(\mathrm{N}_{\mathrm{f}}\right)$ can then be computed by solving any of the above equations provided that the information of the stress and strain is known from the load history. The profiles of stress and strain, which are generated from either straincontrol or stress-control experiments, form a hysteresis loop due to irrecoverable plastic strain under constant amplitude loading. The cyclic stress-strain curve is plotted by connecting the tips of hysteresis loops at different load amplitudes as shown in Figure 4.2, which can be expressed in the following equation [Bannantine et al. (1990)]:

$$
\frac{\Delta \varepsilon}{2}=\frac{\Delta \sigma}{2 E}+\left(\frac{\Delta \sigma}{2 K}\right)^{1 / n^{\prime}}
$$

where $\mathrm{n}^{\prime}$ : cyclic-strain hardening exponent which can be expressed as $\frac{\mathrm{b}}{\mathrm{c}}$;

$K^{\prime}$ : cyclic-strength coefficient which can be expressed as $\frac{\sigma_{f^{\prime}}}{\left(\varepsilon_{f^{\prime}}\right)^{n^{n^{\prime}}}}$;

$\frac{\Delta \sigma}{2 \mathrm{E}}$ is the elastic strain amplitude $\left(\frac{\Delta \varepsilon_{\mathrm{e}}}{2}\right) ;$ and

$\left(\frac{\Delta \sigma}{2 K^{\prime}}\right)^{1 / n^{\prime}}$ is the plastic strain amplitude $\left(\frac{\Delta \varepsilon_{p}}{2}\right)$. 


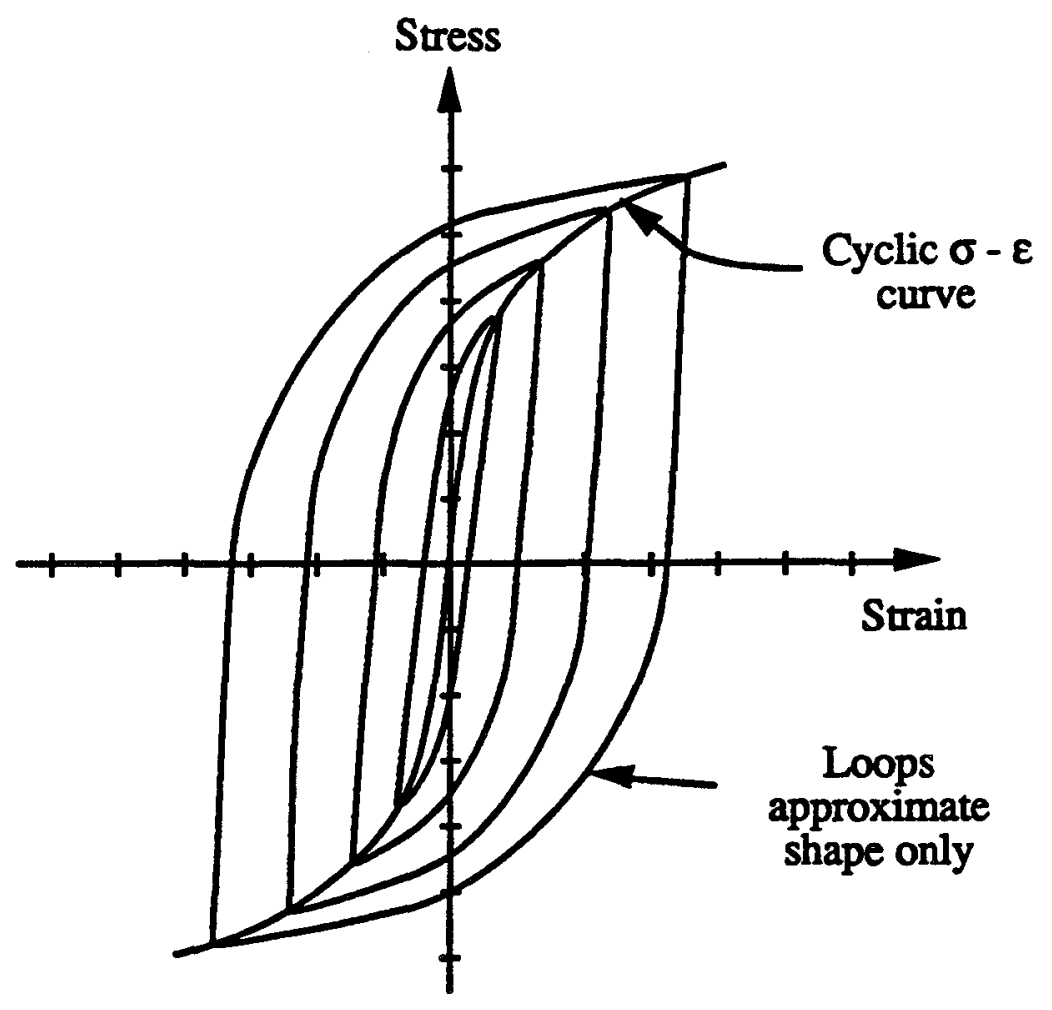

Figure 4.2 Cyclic Stress-Strain Curve

For an unnotched component, the stress range $\Delta \sigma$ can be computed from the dynamic system analysis. The strain range and total cycles to failure are then obtained from eqs.(4.1) to (4.4). In most notched components, however, a simple stress analysis at the notch is usually not possible. Instead, the local strain is first obtained by direct measurement at the notch if possible or by finite element analysis when it is difficult to measure due to complex geometry of the component. The strain is then converted to stress following the cyclic stress-strain characteristics of the specific material.

The structural components under plant operations would usually be subjected to complex loading with varying amplitudes and frequencies. In that case, it becomes more difficult to recognize the cycles since the stress-strain hysteresis loops under spectrum loading are not closed on a cycle-by-cycle basis. To solve this problem, several approaches such as range-pair and rainflow [Fuchs and Stephens (1980)] have been proposed to identify the cycles within the spectrum of the complex load history. All of these approaches attempt to extract the small cycles from the load history without losing the large cycles which significantly contribute to the damage. Since the rainflow cycle counting is widely used in the strain-life approach, it has been adopted in this report for development of a continuous-time damage model. The concept of rainflow cycle counting is briefly explained in Appendix B. Once a cycle is clearly identified, the linear damage of one cycle corresponding to a specific strain amplitude and mean stress is defined as:

$$
D=\frac{1}{N_{f}}
$$


where $\mathrm{N}$ is the total number of cycles to failure at this stress and strain level. The accumulated damage of the load history is assumed to a linear summation of the individual cycles by the Palmgren-Miner's rule [Miner (1945)] as given below :

$$
D=\sum_{\mathbf{i}=1}^{\mathbf{n}} \frac{1}{\mathbf{N}_{\mathbf{n}}}
$$

The fatigue damage is usually assumed to be independent of the frequency of the load and the shape of cyclic stress-strain hysteresis loop. However, when the component is exposed to high temperature, other effects such as creep may have to be taken into account and combined with the fatigue for damage computation. The modeling of fatigue-corrosion-creep is briefly discussed in Appendix E.

\subsection{Continuous-Time Damage Model}

This section introduces the development of a continuous-time damage model based on the strain-life approach as described above. The linear damage accumulation model in eqs. (4.5) and (4.6) is extended to define the damage increment between any two points within a cycle as explained below:

Following Figure 4.3, let the point $O$ be the reference point of a reversal which is determined from rainflow cycle counting. Let $A$ and $B$ be two consecutive points on the same rising reversal, and let $N_{A}$ and $N_{B}$ represent the total number of cycles to failure associated with constant amplitudes, $\mathrm{OA} / 2$ and $\mathrm{OB} / 2$, respectively. Denoting the damage of a rising reversal as $\delta$, the damage increment between the points $A$ and $B$ is defined as:

$$
\Delta \delta=\frac{1}{N_{B}}-\frac{1}{N_{A}}
$$

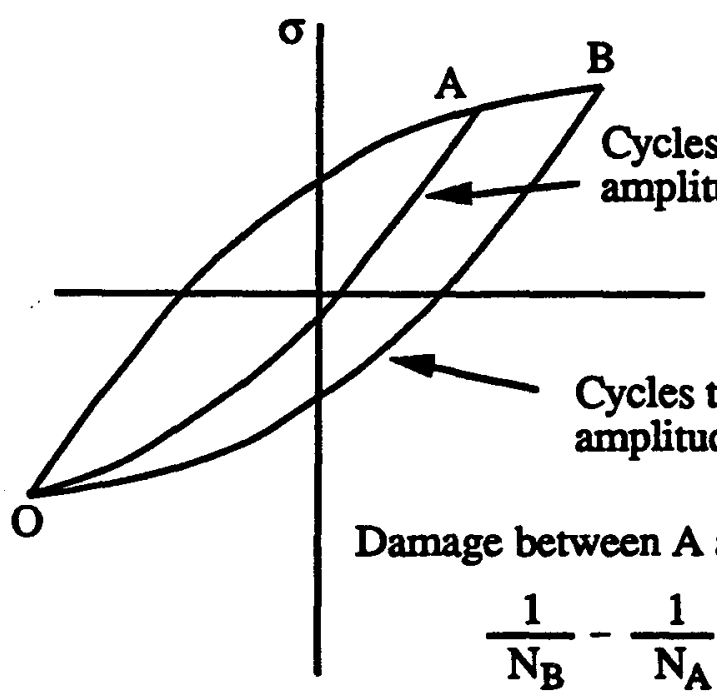

Figure 4.3 Definition of Damage Between Two Points Within a Reversal 
In eq. (4.7), it is assumed that the damage occurs only on the rising reversal, i.e., when the stress is monotonically increasing, and no damage is incurred during unloading, i.e., when the stress is monotonically decreasing. The assumptions are consistent with the physical phenomena observed in the fatigue crack propagation process [Suresh (1991)]. Given that $\Delta \sigma$ is the stress increment between point $A$ and point $B$, the average damage rate with respect to this stress change is equal to $\Delta \delta / \Delta \sigma$. Let $\Delta t$ be the time interval from $A$ to $B$, the average damage rate in terms of the stress can be transformed into the time domain by $\Delta \delta / \Delta t=(\Delta \delta / \Delta \sigma) \times(\Delta \sigma / \Delta t)$. Making $\Delta t$ infinitesimally small, the instantaneous damage rate becomes

$$
\frac{\mathrm{d} \delta}{\mathrm{dt}}=\lim _{\Delta t \rightarrow 0} \frac{\Delta \delta}{\Delta \sigma} \times \frac{\Delta \sigma}{\Delta t}=\frac{\mathrm{d} \delta}{\mathrm{d} \sigma} \times \frac{\mathrm{d} \sigma}{\mathrm{dt}}
$$

where the instantaneous stress rate $d \sigma / d t$ can be generated either from direct measurements of strain rate or from finite element analysis, and $d \delta / d \sigma$ is derived from the existing cycle-based formulae [Bannantine et al. (1990); Dowling (1983)].

In this report, Dowling's life prediction formula of eq. (4.3) and the stress-strain curve of eq. (4.4) are used to evaluate $d \delta / d \sigma$ in eq. (4.8). Replacing $\mathrm{N}_{\mathrm{f}}$ by $1 / \delta$, eq. (4.3) can be written as

$$
\frac{\left|\varepsilon-\varepsilon_{\mathrm{r}}\right|}{2}=\frac{\sigma_{\mathrm{f}}^{\prime}-\sigma_{\mathrm{m}}}{\mathrm{E}}\left(\frac{\delta}{2}\right)^{-\mathrm{b}}+\varepsilon_{\mathrm{f}^{\prime}}\left(1-\frac{\sigma_{\mathrm{m}}}{\sigma_{\mathrm{f}^{\prime}}}\right)^{\frac{\mathrm{c}}{\mathrm{b}}}\left(\frac{\delta}{2}\right)^{-\mathrm{c}}
$$

where $\varepsilon_{\mathrm{T}}$ is the total strain corresponding to the reference stress $\sigma_{\mathrm{T}}$ at the starting point of a given reversal as determined from the rainflow cycle counting method; and $\left|\varepsilon-\varepsilon_{\mathrm{r}}\right| / 2$ is the strain amplitude between the current point and the reference point. The above equation does not provide a closed form solution for the predicted damage $\delta$. The general approach to solve this problem is to separate eq. (4.9) into two different modes. The first term on the right side corresponds to the so called elastic damage mode $\delta_{e}$ and the second term corresponds to the so called plastic damage mode $\delta_{p}$. These two damages, $\delta_{e}$ and $\delta_{p}$, can be derived in an explicit form. For high cycle fatigue, the elastic damage usually yields more accurate prediction than the plastic damage, and vice versa for low cycle fatigue. Generally speaking, the transition between high cycle fatigue and low cycle fatigue is defined as point of intersection of the elastic strain-life curve and plastic strain-life curve as shown in Figure 4.1. In this report, it is proposed that the predicted damage $\delta$ should be obtained as a weighted average of $\delta_{\mathrm{e}}$ and $\delta_{\mathrm{p}}$ where the weights depend on the relative accuracy of the elastic and plastic modes of damage computation under the current load condition.

The elastic strain, $\varepsilon_{\mathrm{re}}$, and the plastic strain, $\varepsilon_{\mathrm{rp}}$ corresponding to the reference stress, $\sigma_{\mathrm{T}}$, are defined as:

$$
\varepsilon_{\mathrm{re}}=\frac{\sigma_{\mathrm{r}}}{\mathrm{E}} \quad \text { and } \quad \varepsilon_{\mathrm{rp}}=\varepsilon_{\mathrm{r}}-\varepsilon_{\mathrm{re}}
$$


Eq.(4.9) can be split into the elastic damage mode and plastic damage mode as:

$$
\begin{aligned}
& \frac{\left|\varepsilon_{\mathrm{e}}-\varepsilon_{\mathrm{re}}\right|}{2}=\frac{\sigma_{\mathrm{f}}^{\prime}-\sigma_{\mathrm{m}}}{\mathrm{E}}\left(\frac{\delta_{\mathrm{e}}}{2}\right)^{-\mathrm{b}} \\
& \frac{\left|\varepsilon_{\mathrm{p}}-\varepsilon_{\mathrm{rp}}\right|}{2}=\varepsilon_{\mathrm{f}}^{\prime}\left(1-\frac{\sigma_{\mathrm{m}}}{\sigma_{\mathrm{f}^{\prime}}}\right)^{\frac{\mathrm{c}}{\mathrm{b}}}\left(\frac{\delta_{\mathrm{p}}}{2}\right)^{-\mathrm{c}}
\end{aligned}
$$

where the elastic and plastic strain amplitudes, $\left|\varepsilon_{\mathrm{e}}-\varepsilon_{\mathrm{re}}\right| / 2$ and $\left|\varepsilon_{\mathrm{p}}-\varepsilon_{\mathrm{rp}}\right| / 2$, respectively, are related to the state of stress following the cyclic stress-strain characteristics of the material as described in eq. (4.4):

$$
\begin{aligned}
& \frac{\left|\varepsilon_{\mathrm{e}}-\varepsilon_{\mathrm{re}}\right|}{2}=\frac{\left|\sigma-\sigma_{\mathrm{r}}\right|}{2 \mathrm{E}} \\
& \frac{\left|\varepsilon_{\mathrm{p}}-\varepsilon_{\mathrm{rp}}\right|}{2}=\left(\frac{\left|\sigma-\sigma_{\mathrm{r}}\right|}{2 \mathrm{~K}^{\prime}}\right)^{\frac{1}{\mathrm{n}^{\prime}}}
\end{aligned}
$$

From eqs. (4.11) and (4.12), the closed form solutions for $\delta_{c}$ and $\delta_{p}$ can be obtained in terms of stress instead of strain as given below:

$$
\begin{aligned}
& \delta_{\mathrm{e}}=2\left(\frac{\left|\sigma-\sigma_{\mathrm{r}}\right|}{2\left(\sigma_{\mathrm{f}}^{\prime}-\sigma_{\mathrm{m}}\right)}\right)^{-\frac{1}{b}} \\
& \delta_{\mathrm{p}}=2\left(\frac{1}{\varepsilon_{\mathrm{f}}^{\prime}}\left(\frac{\left|\sigma-\sigma_{\mathrm{r}}\right|}{2 \mathrm{~K}}\right)^{\frac{1}{\mathrm{n}^{\prime}}}\left(1-\frac{\sigma_{\mathrm{m}}}{\sigma_{\mathrm{f}^{\prime}}}\right)^{-\frac{\mathrm{c}}{\mathrm{b}}}\right)^{-\frac{1}{\mathrm{c}}}
\end{aligned}
$$

Step changes in the reference stress $\sigma_{\mathrm{r}}$ can occur only at isolated points in the load spectrum. Since the damage increment is zero at any isolated point, the damage accumulation can be evaluated at all points excluding these isolated points which constitute a set of zero Lebesgue measure [Royden (1988)]. Exclusion of the points of step changes in $\sigma_{\mathrm{r}}$ does not cause any error in the computation of damage, and $d \sigma_{\mathrm{r}} / \mathrm{dt}$ can be set to zero because $\sigma_{\mathrm{r}}$ is piecewise constant. Furthermore, since it is assumed that no damage occurs during unloading, the damage rate can be made equal to zero when $\sigma<\sigma_{\mathrm{r}}$. Then, the elastic damage rate $\mathrm{d} \delta_{\mathrm{e}} / \mathrm{dt}$ and the plastic damage rate $\mathrm{d} \delta_{\mathrm{p}} / \mathrm{dt}$ are computed by differentiating eqs. (4.13a) and (4.13b) as follows: 
If $\sigma \geq \sigma_{\mathrm{r}}$, then

$$
\begin{aligned}
& \frac{d \delta_{e}}{d t}=2 \frac{d}{d \sigma}\left(\left(\frac{\sigma-\sigma_{\mathrm{r}}}{2\left(\sigma_{f^{\prime}}-\sigma_{\mathrm{m}}\right)}\right)^{-\frac{1}{b}}\right) \frac{d \sigma}{d t}, \text { and } \\
& \frac{d \delta_{p}}{d t}=2 \frac{d}{d \sigma}\left(\left(\frac{1}{\varepsilon_{f^{\prime}}}\left(\frac{\sigma-\sigma_{\mathrm{r}}}{2 K^{\prime}}\right)^{\frac{1}{n^{\prime}}}\left(1-\frac{\sigma_{m}}{\sigma_{f^{\prime}}}\right)^{-\frac{c}{b}}\right)^{-\frac{1}{c}} \frac{d \sigma}{d t} ;\right.
\end{aligned}
$$

else $d \delta_{e} / d t=0$ and $d \delta_{p} / d t=0$.

The damage rate $d \delta / d t$ is obtained as the weighted average of the elastic and plastic damage rates such that

$$
\frac{d \delta}{d t}=w \frac{d \delta_{e}}{d t}+(1-w) \frac{d \delta_{p}}{d t}
$$

where the weighting function, $w$, is selected on the basis of the elastic and plastic strain amplitudes in eqs. (4.11a) and (4.11b) as:

$$
\mathrm{w}=\frac{\varepsilon_{\mathrm{e}}-\varepsilon_{\mathrm{re}}}{\varepsilon-\varepsilon_{\mathrm{r}}} \text { and } 1-\mathrm{w}=\frac{\varepsilon_{\mathrm{p}}-\varepsilon_{\mathrm{rp}}}{\varepsilon-\varepsilon_{\mathrm{r}}}
$$

Eqs. (4.14) to (4.16) are then used to obtain the damage rate at any instant. The damage increment between two consecutive points $t_{k}$ and $t_{k+1}$ on the same reversal can be calculated by integrating the damage differential $\mathrm{d} \delta$. The procedure for evaluation of the accumulated damage is described below:

Assuming that there is no reference point change between $t_{k}$ and $t_{k+1}$, i.e., no small cycle is closed between $t_{k}$ and $t_{k+1}$, the damage increment during this interval is given as:

$$
\begin{aligned}
\delta & =\int_{t_{k}}^{t_{k+1}}\left(w \frac{d \delta_{e}}{d t}+(1-w) \frac{d \delta_{p}}{d t}\right) d t \\
& =w\left(\delta_{e}\left(\sigma\left(t_{k}+1\right), \sigma_{r}\right)-\delta_{e}\left(\sigma\left(t_{k}\right), \sigma_{r}\right)\right)+(1-w)\left(\delta_{p}\left(\sigma\left(t_{k}+1\right), \sigma_{r}\right)-\delta_{p}\left(\sigma\left(t_{k}\right), \sigma_{r}\right)\right)
\end{aligned}
$$

where the weighting function is evaluated at the final point $t_{k+1}$ such that $w=\left(\varepsilon_{e}\left(t_{k+1}\right)-\right.$ $\left.\varepsilon_{\mathrm{re}}\right) /\left(\varepsilon\left(t_{\mathrm{k}+1}\right)-\varepsilon_{\mathrm{r}}\right)$. Furthermore, $w$ is assumed to remain constant in the time interval ( $\mathrm{k}_{\mathrm{k}}$, $\mathrm{t}_{+1}$ ) because the stress increment is sufficiently small during this interval. Nevertheless, the weight, $w$, would have no significant bearing on the damage computation if the difference between $d \delta_{e} / d t$ and $d \delta_{p} / d t$ derived in eqs. (4-14a) and (4$14 \mathrm{~b})$ is small. 
If a change in the reference point occurs at $\tau_{r} \in\left(t_{k}, t_{k+1}\right)$, i.e., if a small cycle is closed at $\tau_{\mathrm{r}}$, then the integral in eq. (4.17) can be split into two parts to exclude the point at $\tau_{\mathrm{r}}$ where a step change in $\sigma_{\mathrm{r}}$ occurs.

$$
\begin{aligned}
\delta & =\int_{\tau_{k}}^{\tau_{\mathrm{r}}}\left(w \frac{d \delta_{e}}{d t}+(1-w) \frac{d \delta_{p}}{d t}\right) d t+\int_{\tau_{r}}^{t_{k+1}}\left(w \frac{d \delta_{e}}{d t}+(1-w) \frac{d \delta_{p}}{d t}\right) d t \\
& =w\left(\delta_{e}\left(\sigma\left(\tau_{r}\right), \sigma_{r}\right)-\delta_{e}\left(\sigma\left(t_{k}\right), \sigma_{\mathrm{r} 1}\right)\right) \\
& +(1-w)\left(\delta_{p}\left(\sigma\left(\tau_{r}\right), \sigma_{\mathrm{r} 1}\right)-\delta_{\mathrm{p}}\left(\sigma\left(t_{\mathrm{k}}\right), \sigma_{\mathrm{r} 1}\right)\right) \\
& +w\left(\delta_{e}\left(\sigma\left(t_{k+1}\right), \sigma_{\mathrm{r} 2}\right)-\delta_{e}\left(\sigma\left(\tau_{\mathrm{r}}\right), \sigma_{\mathrm{r} 2}\right)\right) \\
& +(1-w)\left(\delta_{\mathrm{p}}\left(\sigma\left(t_{\mathrm{k}+1}\right), \sigma_{\mathrm{r} 2}\right)-\delta_{\mathrm{p}}\left(\sigma\left(\tau_{\mathrm{r}}\right), \sigma_{\mathrm{r} 2}\right)\right)
\end{aligned}
$$

where $\sigma_{\mathrm{r} 1}$ is the reference stress associated with the small cycle, and $\sigma_{\mathrm{r} 2}$ is the reference stress associated with the large cycle.. Figure 4.4 shows both stress-time and stress-strain curves when a small cycle is closed between $t_{k}$ and $t_{k+1}$. If more than one cycle is closed during the interval $\left(t_{k}, t_{k+1}\right)$, the integration can be split into as many parts as necessary to exclude those points where the cycles are closed because these points form a set of zero Lebesgue measure as discussed earlier.
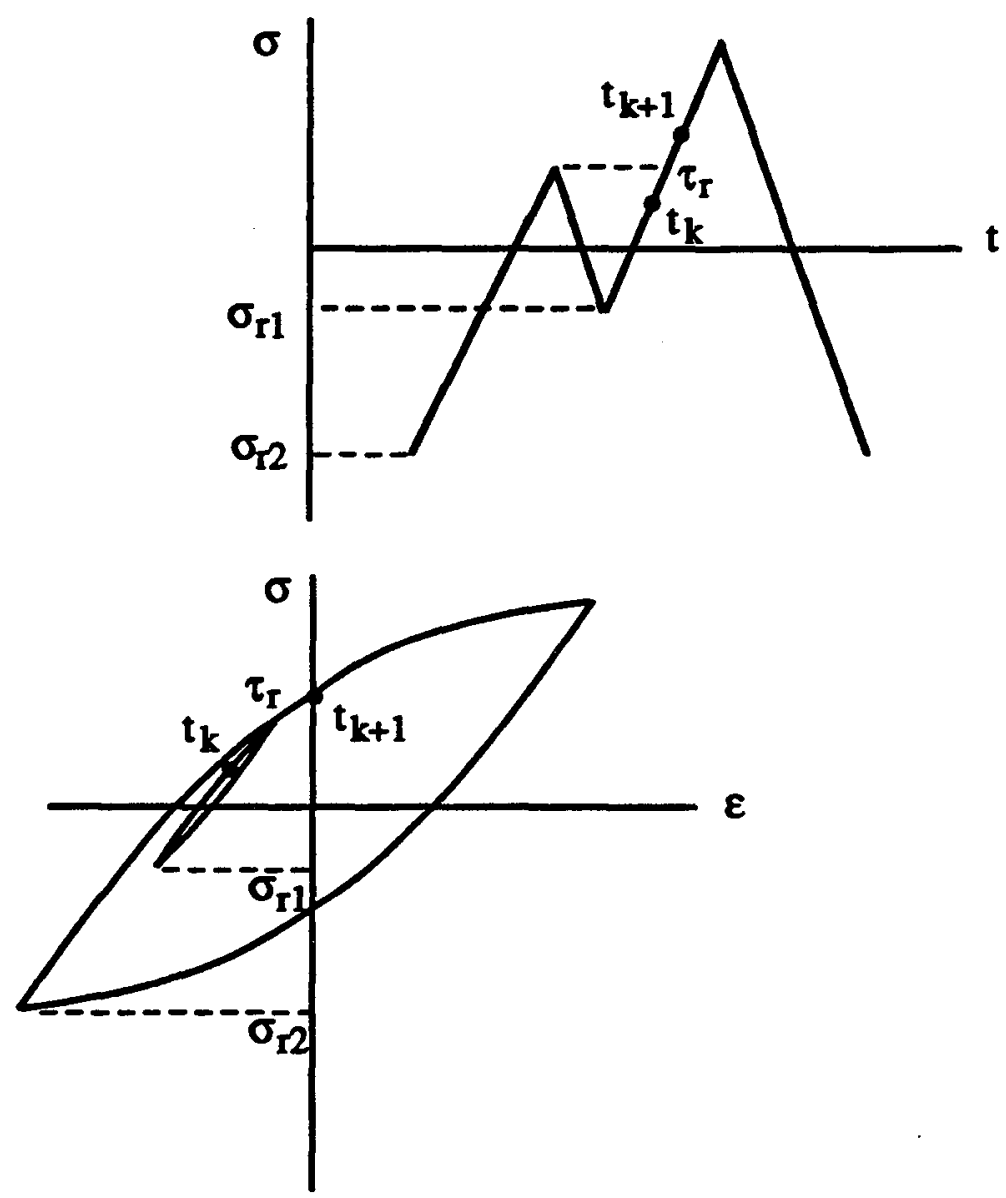

Figure 4.4 Integration When a Cycle Is Closed Between $t_{k}$ and $t_{k+1}$ 


\subsection{Results of Simulation Experiments}

The continuous-time damage model was verified by using the experimental data generated in the SAE cumulative fatigue damage test program [Tucker and Bussa (1977)]. This program was conducted using a notched specimen shown in Figure 4.5, and the materials under tests were Man-Ten and RQC-100 steels which are commonly used in the ground vehicle industry. Table 4.1 lists the fatigue properties for these two materials. The profiles of load spectrum were recorded from the components operating under the actual service condition. Three load profiles, named as suspension, bracket, and transmission, were obtained with compressive, approximately zero, and tensile mean stresses, respectively. Each load profile was normalized such that the absolute value of the maximum load is equal to 999, and small cycles were filtered out of the original history. The notch root strain versus applied load was measured from the strain gauge, and the relationship is plotted in Figure 4.6. The applied load is converted into local strain following Figure 4.6 and the local stress is calculated from the cyclic stressstrain curve in eq. (4.4). The continuous-time damage model developed in section 4.2 is then used to predict the accumulated damage and damage rate. Three different load levels of the transmission history are simulated based on the Man-Ten steel data, and the results are shown in Figure 4.7, 4.8 and 4.9. The damages computed from the strain-life approach are also included for comparison. In the results obtained by the strain-life approach, only those points on the peak of a cycle are evaluated, and the damages of reversals are computed whenever cycles are not closed.

Table 4.1 Smooth Specimen Stress-Strain and Fatigue Properties

\begin{tabular}{|l|c|c|}
\hline \multicolumn{1}{|c|}{ Property } & Man - Ten & RQC - 100 \\
\hline Modulus of Elasticity, E & $203,000 \mathrm{Mpa}$ & $203,000 \mathrm{Mpa}$ \\
Cyclic Strain Hardening Exponent, $\mathrm{n}^{\prime}$ & 0.193 & 0.100 \\
Cyclic Strength Coefficient, K' & $1190 \mathrm{Mpa}$ & $1150 \mathrm{Mpa}$ \\
Fatigue Strength Coefficient, $\sigma^{\prime}$ & $930 \mathrm{Mpa}$ & $1165 \mathrm{Mpa}$ \\
Fatigue Strength Exponent, b & -0.095 & -0.075 \\
Fatigue Ductility Coefficient, $\varepsilon^{\prime}$ & 0.26 & 1.06 \\
Fatigue Ductility Exponent, c & -0.47 & -0.75 \\
\hline
\end{tabular}

As seen in Figure 4.7, 4.8 and 4.9, the predicted damage generated from the continuous-time damage model agrees closely with that from the strain-life approach. Each figure shows the accumulated damage of one block load history which contains 1708 reversals. For high cycle fatigue, i.e., maximum load $=15.6 \mathrm{KN}$, the continuoustime damage model tends to predict slightly higher damage than the strain-life approach. For low cycle fatigue, i.e., maximum load $=71.2 \mathrm{KN}$, the continuous-time damage model predicts less damage. The relative error, which is defined as the difference of damage increments between the two approaches divided by the damage increment from the strain-life approach, is within 10\% for almost all the reversals in the load histories for the two cases where the maximum loads are equal to $71.2 \mathrm{KN}$ and $35.6 \mathrm{KN}$. For the maximum load of $15.6 \mathrm{KN}$, the error is higher since the damage computation is more sensitive to a small change of stress or strain in the case of high cycle fatigue but the mean value is nearly zero. The results from both approaches are considered to be in fair agreement in view of scattering of the fatigue test data. 


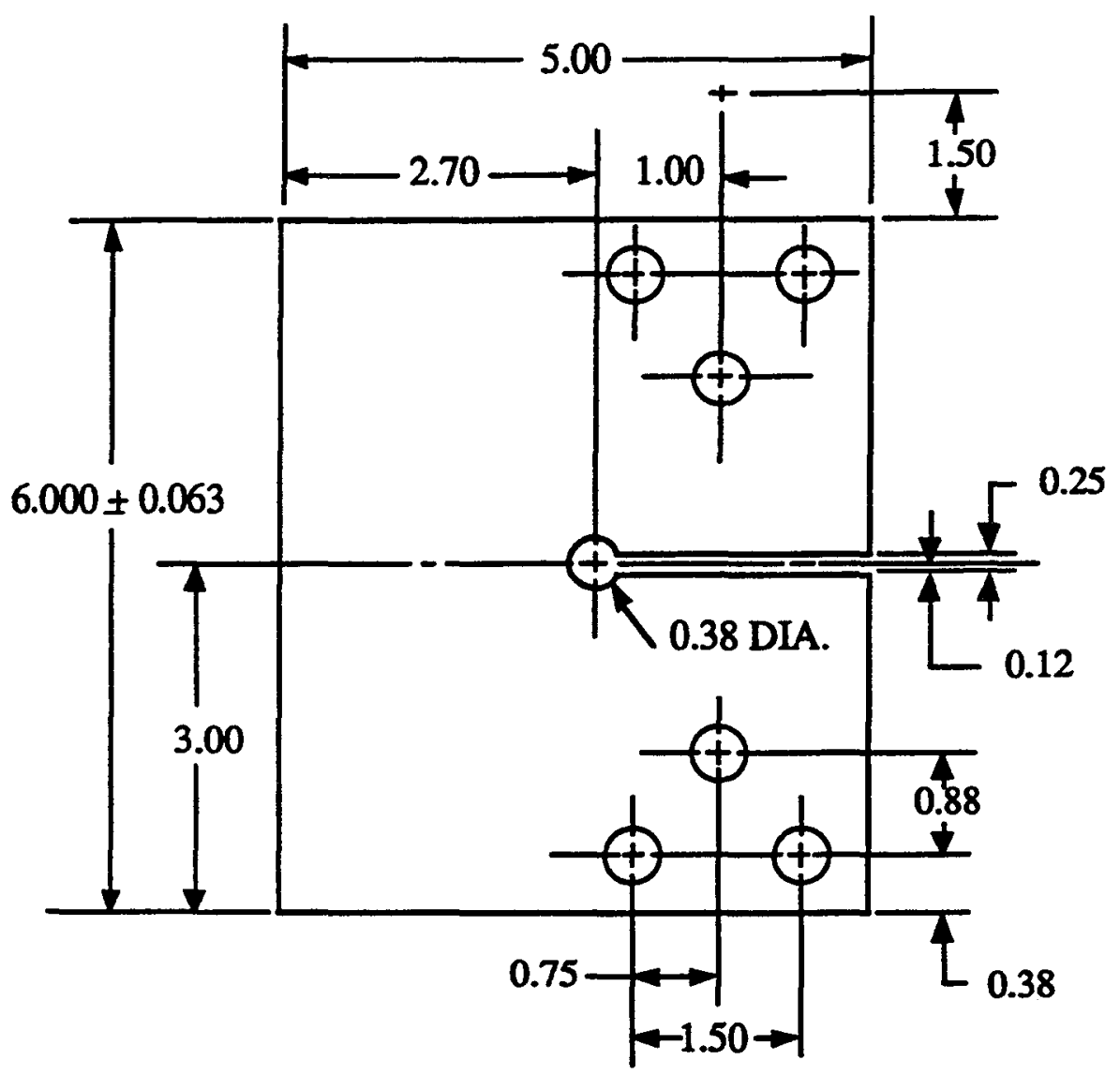

Figure 4.5 Test Specimen Design

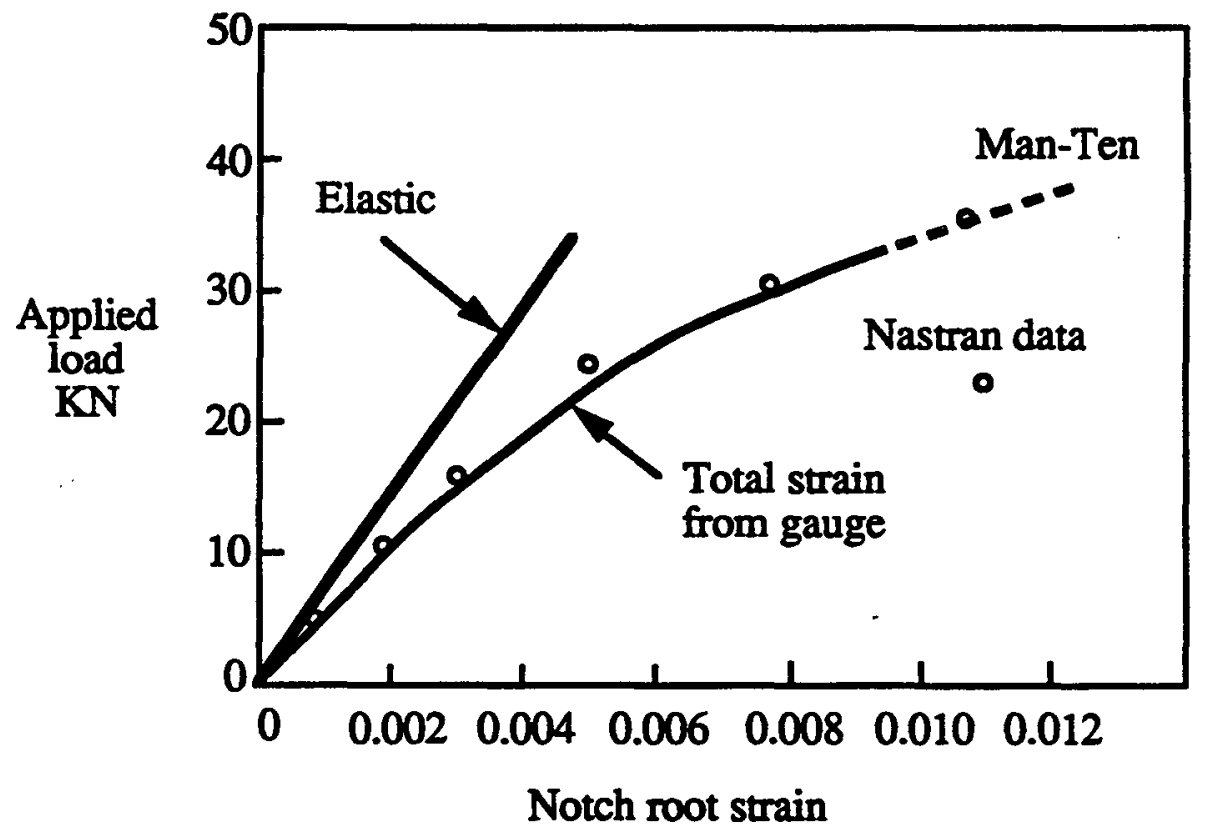

Figure 4.6 Comparison of Measured and Calculated Strain 

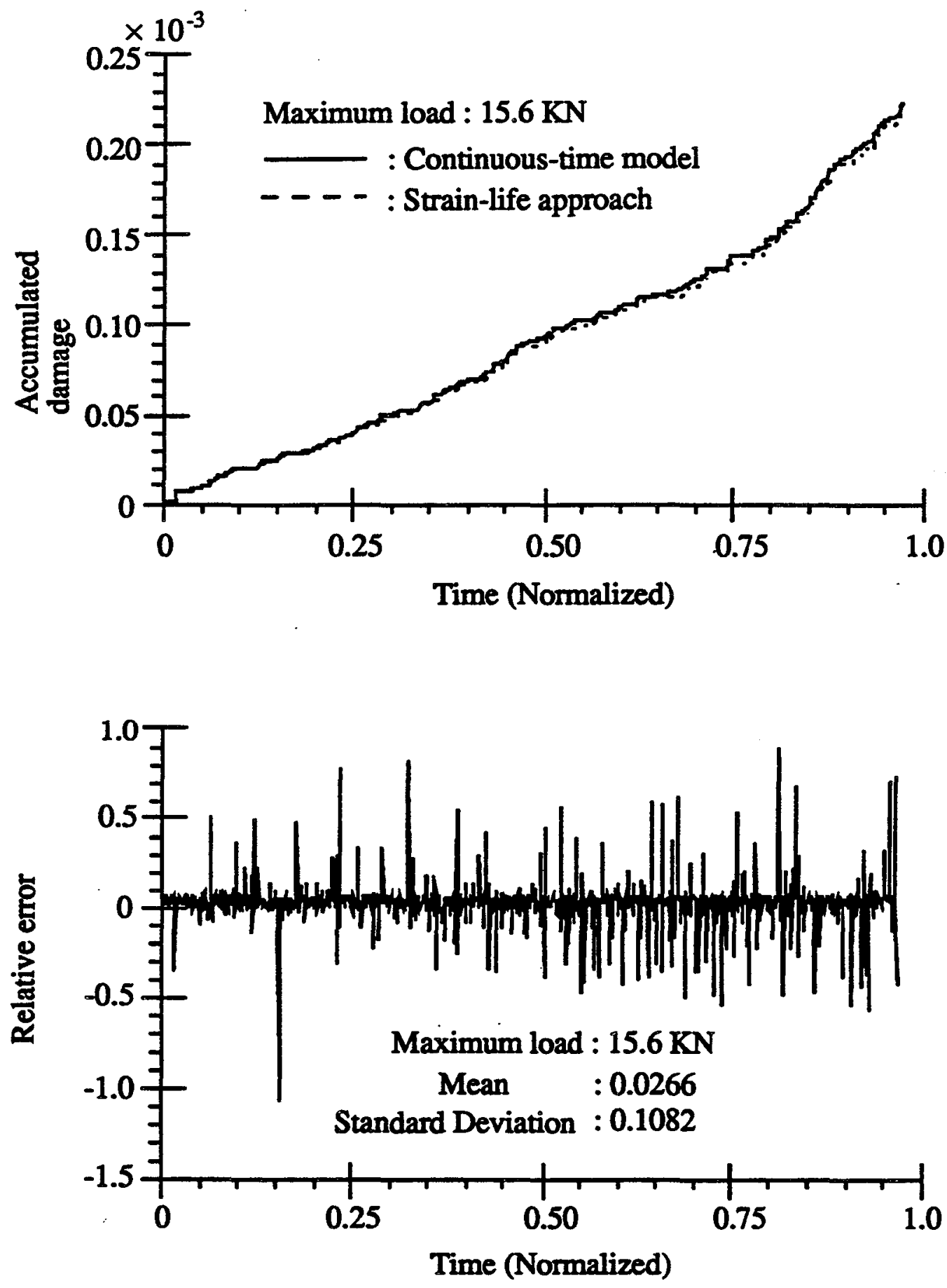

Figure 4.7 A Comparison of Continuous-Time Damage Model with the Strain Life Approach for Maximum Load $=15.6 \mathrm{KN}$. 

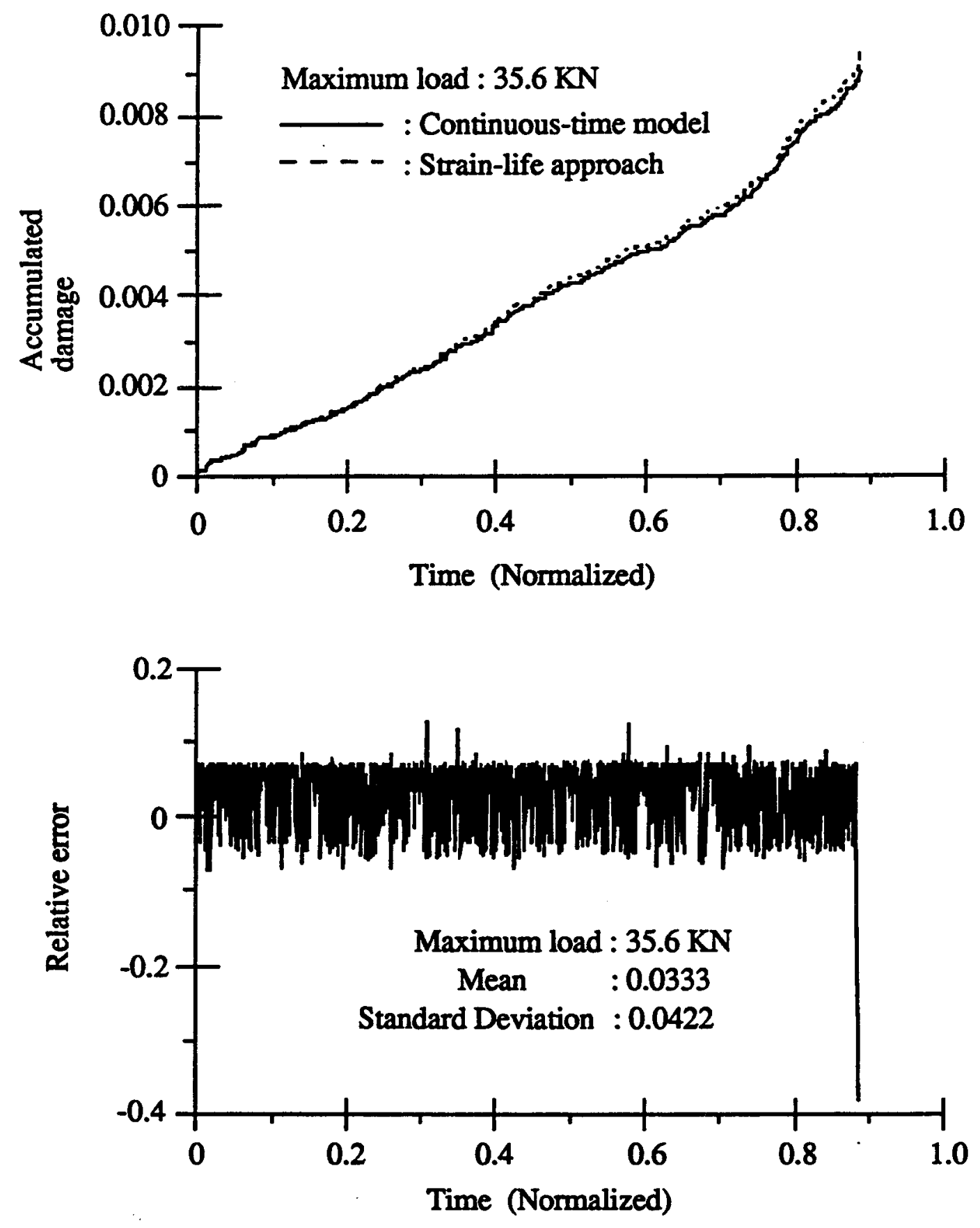

Figure 4.8 A Comparison of Continuous-Time Damage Model with the Strain Life Approach for Maximum Load $=35.6 \mathrm{KN}$ 

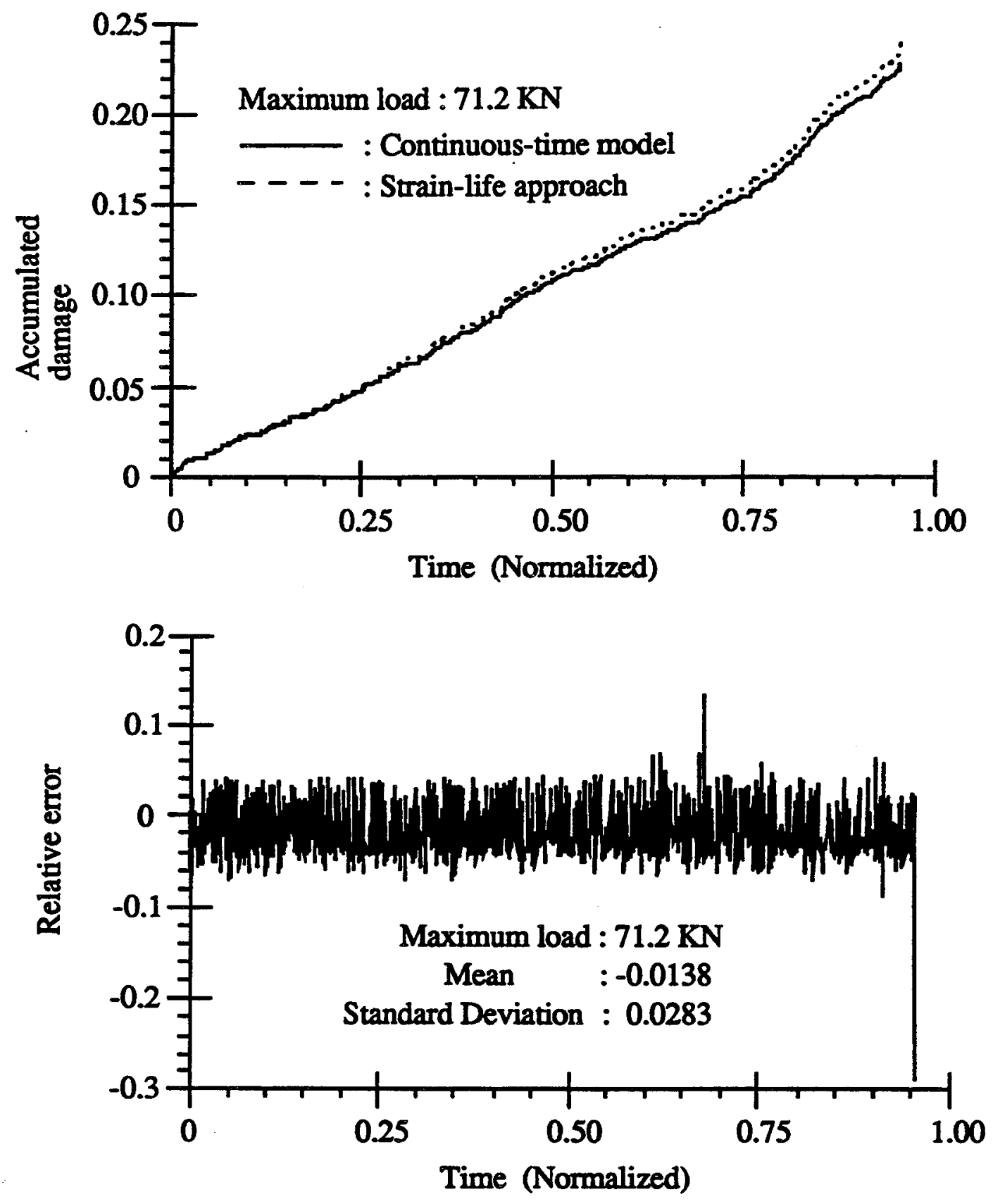

Figure 4.9 A Comparison of Continuous-Time Damage Model with the Strain Life Approach for Maximum Load $=71.2 \mathrm{KN}$ 


\subsection{Modeling of Nonlinear Cumulative Damage Using Damage Curve Approach}

The continuous-time damage model developed above is based on the linear damage accumulation following the Palmgren-Miner's rule. Although this concept of linear damage accumulation has been widely used due to its simplicity in computation, the cumulative damage behavior is actually nonlinear [Suresh (1991)]. Experimental results show that the linear damage rule predicts less damage if a few cycles of high stress are applied before testing with low stress. This phenomenon is known as the sequence effect. In many engineering applications, the components are usually subjected to loading with varying amplitudes. Due to this sequence effect, the linear rule of damage accumulation, which is commonly used for fatigue life assessment, could lead to erroneous results. A nonlinear cumulative damage representation needs to be established for more accurate life prediction of the critical components.

The concept of a nonlinear damage curve to represent the damage was first conceived by Marco and Starkey (1954). No mathematical representation of a damage curve was proposed at that time because the physical process of damage accumulation was not adequately understood. Manson and Halford (1981) proposed the double linear rule primarily based on the damage curve approach for treating cumulative fatigue damage. In their paper, an effort was made to mathematically represent the damage curve and approximate it by two piecewise line segments. The total fatigue life was thereby divided into two phases so that the linear damage rule could be applied in each phase of the life. A concept similar to the damage curve approach was proposed by Bolotin (1989) with a mathematical representation which does not necessarily assess the damage on the basis of cycles and is more appropriate for modeling in the continuous-time setting. Therefore, Bolotin's approach is adopted in this research for the development of a continuous-time model with nonlinear damage accumulation. A review of the damage curve approach following Bolotin's approach is presented below.

Figure 4.10 shows a comparison of the accumulations of linear damage, $D_{\ell}$, and nonlinear damage, $D$, as a function of the cycle ratio, $n / N$, where $n$ is the actual number of cycles undergone and $\mathrm{N}$ is the number of cycles to failure under a constant amplitude load. The damage accumulates along the curve as the loading cycles are applied. For example, if a specimen is subjected to $n_{1}$ cycles of a constant stress amplitude, for which the fatigue life is $N_{1}$ cycles, the accumulated damage will be $D_{a}$ indicated in Figure 4.10, where the abscissa is the normalized cycle ratio with respect to its fatigue life and the ordinate is the damage accumulation of the specimen. Bolotin used the following analytical relationship between $\mathrm{D}$ and $\mathrm{D}_{\ell}$ :

$$
D=\left(D_{\ell}\right)^{\gamma\left(\sigma_{2}\right)}
$$

where the exponent $\gamma$ describes the nonlinearity of the curve and usually is a function of the stress amplitude $\sigma_{\mathrm{a}}$.

Eq. (4.19) indicates that the nonlinear damage may accumulate along different curves under different stress amplitudes. Generally speaking, for high-strength materials that usually strain soften [Hertzberg (1989)], a smaller load amplitude tends to make the damage curve more nonlinear, i.e., increase the $\gamma$-parameter. To realize the effects of nonlinear damage accumulation, consider a smooth specimen be subjected to 
two levels of cyclic loading, $\Delta \sigma_{1}$ and $\Delta \sigma_{2}$ (say $\Delta \sigma_{1}>\Delta \sigma_{2}$ ), with respective fatigue lives of $N_{1}$ and $N_{2}$ cycles $\left(N_{1}<N_{2}\right)$. If $\Delta \sigma_{1}$ is applied for $n_{1}$ cycles followed by $\Delta \sigma_{2}$ for $n_{2}$ cycles when the specimen fails, then the linear rule would underestimate the damage, i.e., $\left(n_{1} / N_{1}+n_{2} / N_{2}\right)<1$. Next consider the reverse situation where $\Delta \sigma_{2}$ is applied first for $n_{2}^{\prime}$ cycles followed by $\Delta \sigma_{1}$ for $n_{1}^{\prime}$ cycles to failure of the specimen. The linear rule, in this case, overestimates the damage, i.e., $\left(n_{1} / N_{1}+n_{2} / N_{2}\right)>1$. Suppose $O X Z$ and $O Y Z$ are the nonlinear damage curves for cyclic loads, $\Delta \sigma_{1}$ and $\Delta \sigma_{2}$ respectively, as shown in Figure 4.11. First, let $n_{1}$ cycles of $\Delta \sigma_{1}$ be applied prior to $\Delta \sigma_{2}$. As the load cycles are applied, the accumulated damage follows the curve $\mathrm{OXZ}$ and reaches the point $A$ with its value equal to $D_{a}$. Then, let $\Delta \sigma_{2}$ be applied until the component fails. The damage accumulation continues from its present state $D_{a}$ along the curve OYZ until the nonlinear damage $D$ reaches 1 . That is, the damage accumulates from point $B$ to $Z$. Suppose the number of cycles needed for the component to fail under $\Delta \sigma_{2}$ is $n_{2}$. As indicated in Figure 4.11, the linear rule underestimates the damage by the length $A B$, which is equal to $1-n_{1} / N_{1}-n_{2} / N_{2}$. In the reverse case, the linear rule overestimates the damage by the length of $A^{\prime} B^{\prime}$ as shown in Figure 4.12.

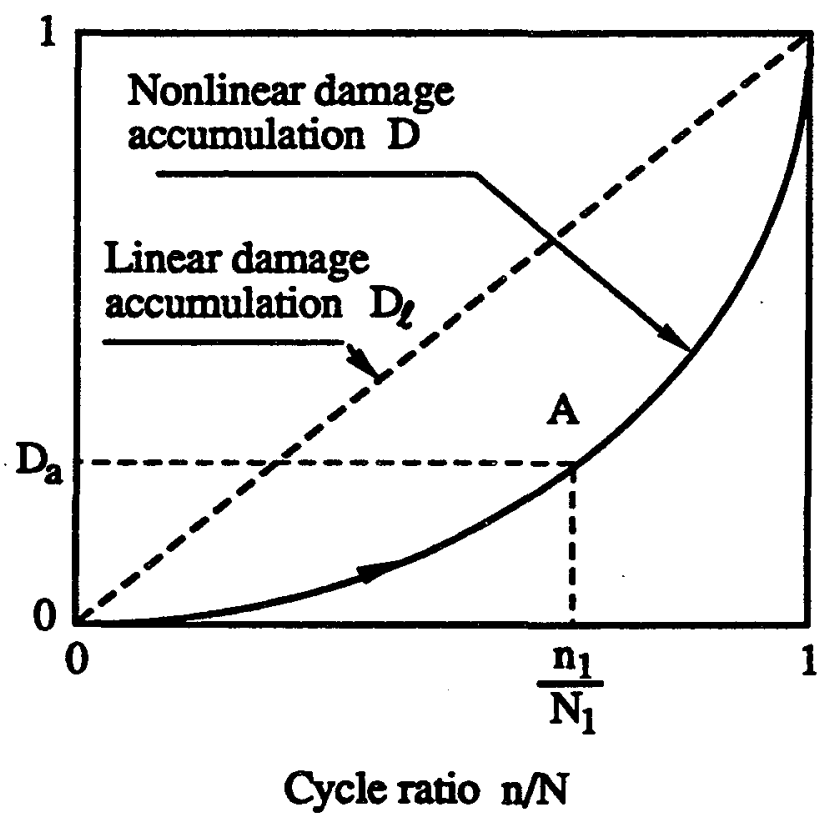

Figure 4.10 Nonlinear Damage Curve under Constant Amplitude Loading

The above example illustrates the basic concept of the damage curve approach for two-level loading. In the case of multiple-level loading, the same procedure can be applied by identifying the present damage state and following the damage curve associated with the current loading condition. If a component is subjected to spectral loading, then the techniques of cycle-counting, prediction of linear damage increments and computation of nonlinear damage via the damage curve approach need to be integrated into a single procedure. 
In the damage curve approach described above, the $\gamma$-parameter is usually assumed to be dependent solely on the stress amplitude level [Manson and Halford (1981)]. Highstrength materials such as $\mathbf{4 3 4 0}$ steel usually yield very large values of $\gamma$ especially under high-cycle fatigue. Manson (1981) made an attempt to fit the $\gamma$-parameter in a damage model that is structurally similar to eq. (4.19) and includes an initial damage term.

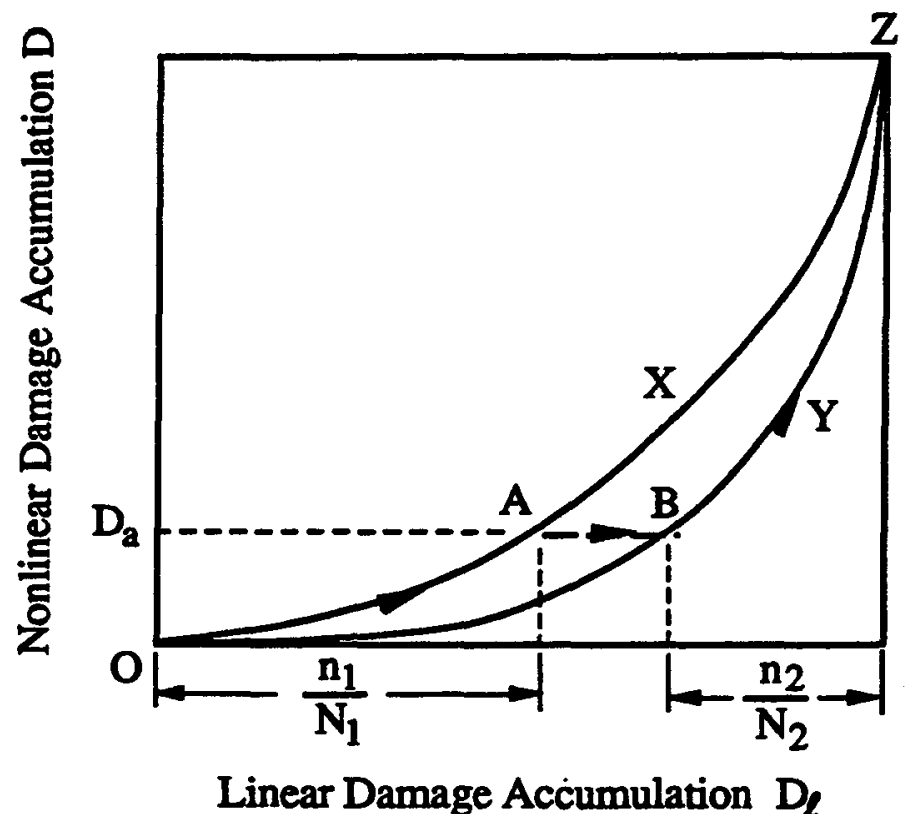

Figure 4.11 Nonlinear Damage Accumulation $\left(\Delta \sigma_{1}>\Delta \sigma_{2}\right)$

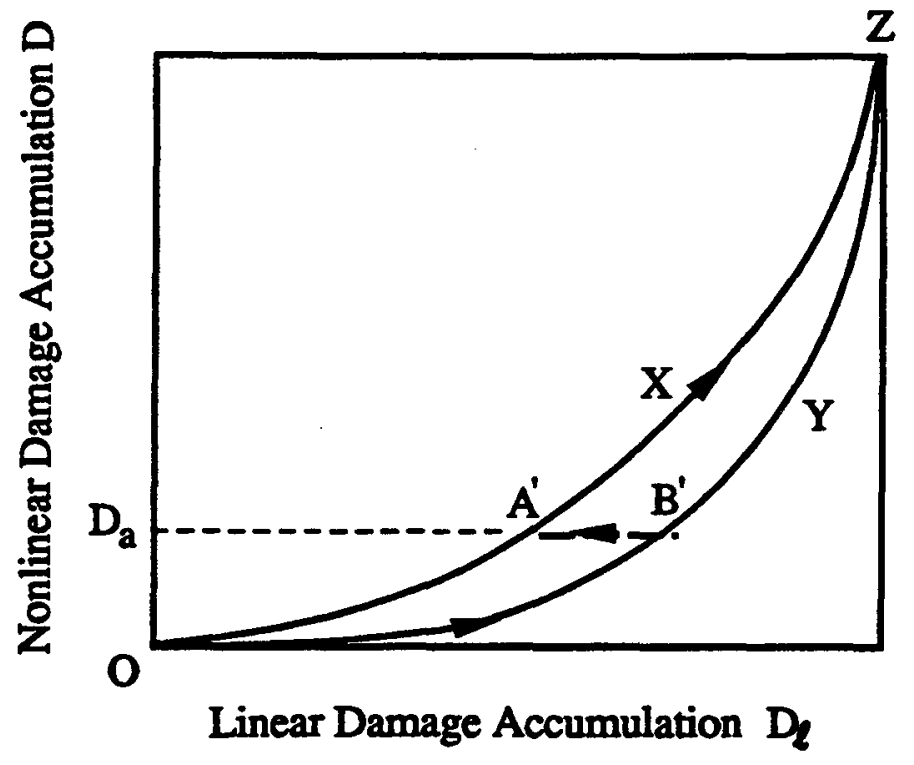

Figure 4.12 Nonlinear Damage Accumulation $\left(\Delta \sigma_{1}<\Delta \sigma_{2}\right)$ 
The $\gamma$-parameter was found to have the following form: $\gamma=(2 / 3) \mathrm{N}_{\mathrm{f}} 0.4$, where $\mathrm{N}_{\mathrm{f}}$ is the number of cycles to failure under a constant load amplitude. For example, if $\mathrm{N}_{\mathrm{f}}$ is assumed to be $10^{5}$ cycles, the $\gamma$-parameter is then equal to 66.7 which appears to be extremely high especially for damage computation when $D<<1$. It also follows from eq. (4.19) that a large $\gamma$, at an early stage of fatigue life, shall yield a very small damage which could be out of the range of the computer precision. This necessitates the formulation of a computationally practical method of damage prediction.

The cause of large $\gamma$-parameter is that, in the conventional damage curve representation as shown in eq. (4.19), the values of $\gamma$ are assumed to be constant at certain stress amplitudes. These curves do not accurately describe the nonlinearity of damage accumulation process for the entire fatigue life of the component. It follows from a crack propagation model such as the Paris model [Paris and Erdogan (1963)] that the crack growth rate is dependent not only on the stress amplitude but also on the current crack length. Recognizing the fact that the crack itself is an index of accumulated damage, it is reasonable to assume the $\gamma$-parameter to be dependent on both stress amplitude and the current level of damage accumulation., i.e., $\gamma=\gamma\left(\sigma_{a}, D\right)$. Therefore, eq. (4.19) should be modified as

$$
D=\left(D_{\ell}\right)^{\gamma\left(\sigma_{2}, D\right)}
$$

where $\mathrm{D}$ and $\mathrm{D}_{\ell}$ are the current states of nonlinear and linear damage accumulation, respectively. Although the above eq. (4.20) has an implicit structure, it can be solved via a recursive relationship.

The next part of this section describes a modification of the damage curve approach to develop a nonlinear damage model in the continuous-time setting. Following the concept of the linear damage model in the continuous-time setting, the nonlinear damage at any point on a rising reversal can be obtained as explained below.

Referring to the bottom part of Figure 4.13 , let $\mathrm{A}$ be any point on the rising reversal and $R$ be its reference point as determined from the rainflow cycle counting method [Dowling (1983)]. Let the current state of damage at the reference point $R$ be equal to $D_{r}$ and let ORAZ be the damage curve associated with the stress amplitude $\mathrm{RA} / 2$ as shown in the top part of Figure 4.13. Corresponding to the nonlinear damage $D_{r}$, the notion of the "virtual" linear damage, $D_{\ell a}$, is brought in as follows:

$$
D_{\ell a}=\left(D_{r}\right)^{\frac{1}{\gamma_{r}^{2}}}
$$

where $\gamma_{\mathrm{r}}^{\mathrm{a}}$ is the $\gamma$-parameter associated with the stress amplitude RA/2 and nonlinear accumulated damage $D_{r}$ at point $R$. The term "virtual" means that $D_{\ell a}$ is the linear damage which would be incurred if the component had been subjected to the cyclic stress of constant amplitude, RA/2, from its initial damage state to the current damage state at $R$. Similar to $\gamma_{\mathrm{r}}^{\mathrm{a}}$ in eq. (4.21), the $\gamma$-parameter associated with the damage state 
$D_{a}$ at the point $A$ is defined as $\gamma_{a}^{a}$. Referring to Figure 4.13, the functional relationships among $D_{a}, D_{r}$ and the corresponding $\gamma$-parameters $\gamma_{a}^{a}$ and $\gamma_{r}^{a}$ are defined as follows:

$$
\left\{\begin{array} { l } 
{ \gamma _ { \mathrm { a } } ^ { \mathrm { a } } = \gamma ( \sigma _ { \mathrm { a } } , \mathrm { D } _ { \mathrm { a } } ) } \\
{ \mathrm { D } _ { \mathrm { a } } = ( \mathrm { D } _ { \ell \mathrm { a } } + \Delta \mathrm { D } _ { \ell \mathrm { a } } ) ^ { \gamma _ { \mathrm { a } } ^ { \mathrm { a } } } }
\end{array} \quad \text { and } \quad \left\{\begin{array}{l}
\gamma_{\mathrm{r}}^{\mathrm{a}}=\gamma\left(\sigma_{\mathrm{a}}, \mathrm{D}_{\mathrm{r}}\right) \\
\mathrm{D}_{\mathrm{r}}=\left(\mathrm{D}_{\ell \mathrm{a}}\right)^{\gamma_{\mathrm{r}}^{\mathrm{a}}}
\end{array}\right.\right.
$$

where $\sigma_{\mathrm{a}}$ is the stress amplitude $R A / 2$, and $\Delta D_{\ell \mathrm{a}}$ is the linear damage increment between $R$ and $A$ obtained via the procedure described in Section 4.2. The damage $D_{T}$
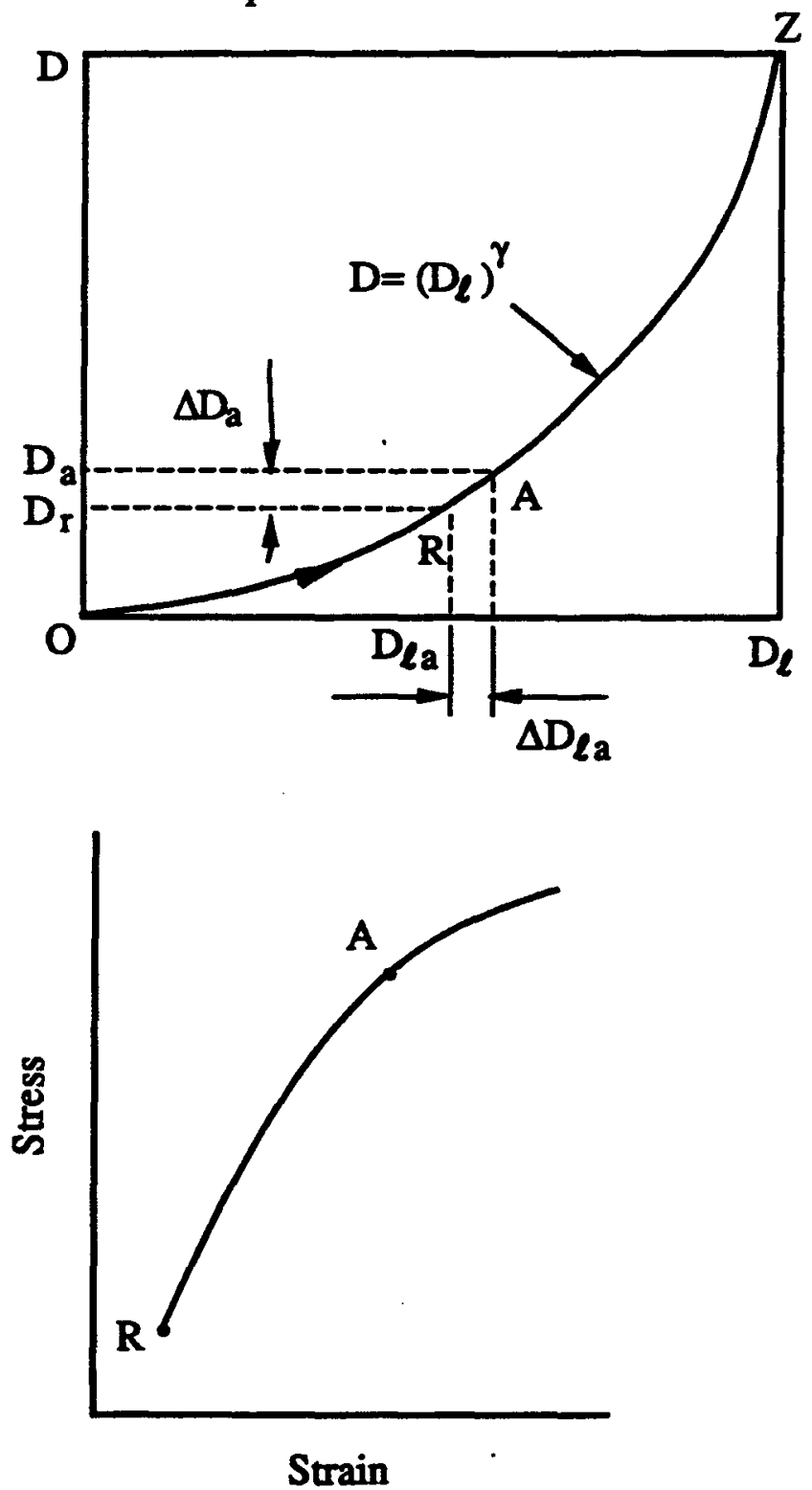

Figure 4.13 Nonlinear Damage Increments 
in the right part of eq. (4.22) represents the damage state at the reference point $R$ and therefore its value is already computed from the past load history. Having known $D_{r}$, the $\gamma_{\text {-parameter }} \gamma_{r}^{a}$ and the "virtual" linear damage $D_{\ell_{a}}$ at $R$ for the stress amplitude $R A / 2$ can be evaluated from the right hand part of eq. (4.22). The two unknowns, $D_{a}$ and $\gamma_{\mathrm{a}}^{\mathrm{a}}$, which represent the damage state and the $\gamma$-parameter at the current point $A$, are computed by solving the equation pair in the left part of eq. (4.22). Now, the (nonlinear) damage increment from the point $R$ to $A$ in Figure 4.13 can be computed as:

$$
\Delta D_{a}=D_{a}-D_{r}=\left(D_{\ell a}+\Delta D_{\ell a}\right)^{\gamma_{a}^{a}}-D_{\ell a} \gamma_{r}^{a}
$$

In summary, the accumulated damage at any point within a reversal can be obtained by solving the following nonlinear equations:

$$
\begin{aligned}
\gamma & =\gamma\left(\sigma_{\mathrm{a}}, \mathrm{D}\right) \\
\mathrm{D} & =\left(\mathrm{D}_{\ell}+\Delta \mathrm{D}_{\ell}\right)^{\boldsymbol{\gamma}}
\end{aligned}
$$

where $D_{\ell}$ is the "virtual" linear damage at the reference point and $\Delta D_{\ell}$ is the linear damage increment for the stress amplitude $\sigma_{\mathrm{a}}$ relative to the reference point.

The pair of equations (4.24) and (4.25) does not have a closed form solution and therefore needs to be solved by an iterative method. However, an iterative solution at every point in the load history may not be practical from the perspective of computational efficiency. An efficient approach of obtaining a numerical solution would operate on a set of discrete points in the load history such that there is only a very small increment of damage between two consecutive points. Thus, both eqs. (4.24) and (4.25) can be treated as linear line segments between the points $R$ and A. This assumption is valid during the entire loading history with the possible exception of very low-cycle fatigue. One more interesting observation is that the $\gamma$-parameter is generally a monotonically increasing function of the nonlinear damage $\mathrm{D}$. This can be interpreted from the Paris equation that the growth rate of a macrocrack becomes larger as the crack length increases. Therefore, the damage rate would be larger at a higher degree of nonlinearity, which implies a larger value of $\gamma$. This phenomenon, however, may not be true if the stress intensity factor range is below the long crack threshold or if the material strain hardens. As seen in Figure 4.14, for the general case of $\gamma>1$ (e.g., high strength materials that usually strain soften [Hertzberg (1989)]), $\gamma$ is a monotonically increasing function of $D$ in eq. (4.24), and $D$ is a monotonically decreasing function of $\gamma$ in eq. (4.25). On the other hand, if $\gamma$ is less than 1 (e.g., ductile materials that usually strain harden), the characteristics of both eqs. (4.24) and (4.25) could be reversed.

Having computed the linear damage $D_{l}$ and linear damage increment $\Delta D_{l}$, a procedure for solving eqs. (4.24) and (4.25) to obtain the nonlinear damage, D, is described below:

1. Let $D_{1}=D_{r}$ and $\gamma_{1}=\gamma_{r}$.

2. Find $D_{2}=\left(D_{\ell}+\Delta D_{\ell}\right)^{\gamma_{1}}$ from eq. (4.25) and $\gamma_{2}=\gamma\left(\sigma_{\mathrm{a}}, D_{2}\right)$ from eq. (4.24) 
3. Compute $\gamma_{3}$ as $\gamma_{3}=\gamma_{1} \times \frac{\log D_{\ell}}{\log \left(D_{\ell}+\Delta D_{\ell}\right)}$ from eqs. (4.20) and (4.24)

4. Find $D_{a}$ in Figure 4.14, which is approximated as the point of intersection of two straight line segments:

$$
\mathrm{D}_{\mathrm{a}}=\frac{\mathrm{D}_{1}\left(\gamma_{2}-\gamma_{1}\right)+\mathrm{D}_{2}\left(\gamma_{3}-\gamma_{1}\right)}{\gamma_{3}+\gamma_{2}-2 \gamma_{1}}
$$

The above procedure computes the nonlinear accumulated damage at any point on the rising reversal. If the stress is monotonically decreasing, there is no damage

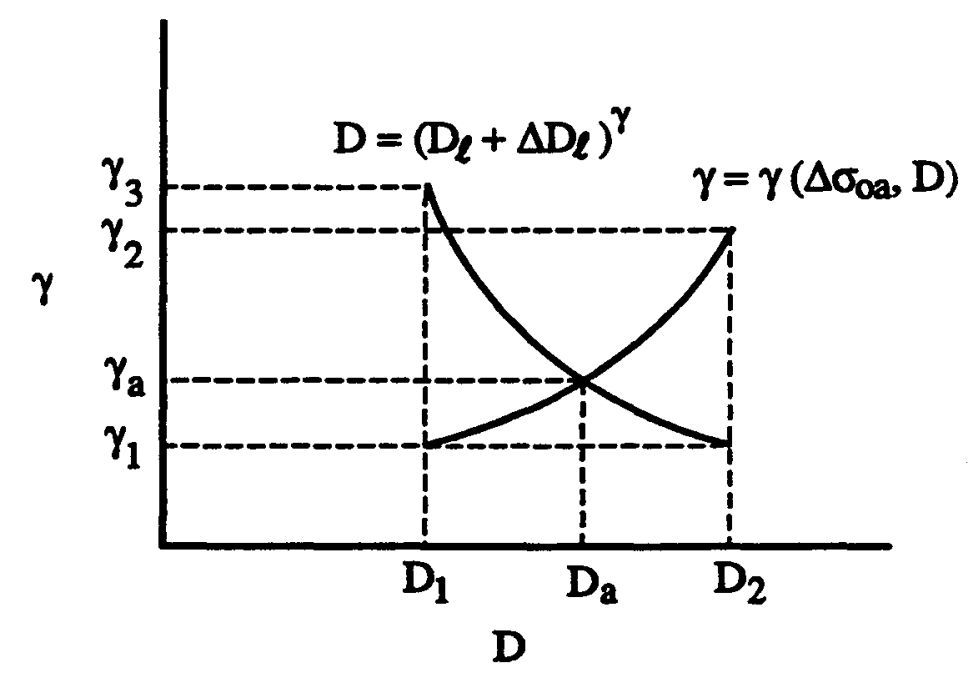

Figure 4.14 Computation of Nonlinear Cumulative Damage

increment as described in eqs. (4.14) and (4.15) for the case of linear damage accumulation. Finally, the rate of nonlinear damage accumulation is obtained directly by differentiating eq. (4.20) with respect to time t:

$$
\frac{d \mathrm{D}}{\mathrm{dt}}=\gamma\left(\mathrm{D}_{\ell}\right)^{\gamma-1} \times \frac{\mathrm{dD} \ell}{\mathrm{dt}}+\left(\mathrm{D}_{\ell}\right)^{\gamma} \ln \mathrm{D}_{\ell} \times \frac{\mathrm{d} \gamma}{\mathrm{dt}} .
$$

\subsection{The $\boldsymbol{\gamma}$-Parameter Fitting for the Nonlinear Cumulative Damage Model}

One major task in the above approach is to identify a mathematical representation for the $\gamma$-parameter as a function of the applied stress amplitude and the current damage state. It requires the knowledge of physical process of damage accumulation which may be obtained from either experimental data or a combination of experimentation and analysis with an appropriate definition of damage. The $\gamma$ parameters have different values, in general, for different materials and follow different equation structures. Furthermore, because the mechanisms attributed to the damage 
accumulation at various stages of fatigue life are different, no single approach can provide accurate assessment of damage throughout the fatigue life of a component. It is difficult, if not impossible, to construct a single structure for representation of $\gamma$. An alternative approach is to evaluate $\gamma$ by interpolation. Once the damage is appropriately defined and an analytical method is selected, the information needed for the nonlinear damage model can be generated via experimentation or analysis for constant stress amplitudes. The values of $\gamma$ are then computed by eq. (4.20) for a given stress level and the damage data ranging from $D_{0}$ (initial damage state) to the failure condition at $D=1$. These generated data, $\gamma$ versus $D$, are then plotted for various amplitudes of stresses. These curves can be either fitted by nonlinear equations, if possible, or as linear piecewise representations. Since it is not practical to perform experiments at infinitesimally small increments of stress amplitude, the values of $\gamma$ can be only experimentally determined at selected discrete levels of stress amplitude. The values of $\boldsymbol{\gamma}$ for other stress amplitudes can then be interpolated. Since the characteristics of $\gamma_{\text {may }}$ strongly depend on the type of the material, availability of pertinent experimental data for the correct material is essential for the damage-mitigating control synthesis. The remaining part of this section presents the results of $\gamma$-parameter fitting based on the experimental data [Swain et al. (1990)] for the material AISI 4340 steel.

In the model of Newman et al. (1981), the stress intensity range, $\Delta \mathrm{K}$, used in the Paris equation is replaced by effective stress intensity range $\Delta K_{\text {eff. }}$ The crack opening stress is determined by a crack closure model which is similar to the Dugdale model [Dugdale (1960)] but it is modified to leave plastically deformed material in the wake of the advancing crack tip. In this simulation, however, the crack opening stress is computed by simplified equations [Newman (1984)] which are obtained through curve fitting based on the original model. The experiments show that a unified approach [Newman et al. (1992)] based on the crack closure concept can be used for damage prediction starting from the initial defect (microcrack) to the failure of materials without significant errors. A relationship between the effective stress intensity factor range and crack growth rate obtained from the experimental data of AISI 4340 is given in Table 4.2 [Swain et al. (1990)].

Table 4.2 Effective Stress Intensity Factor Range versus Crack Growth Rate Relationship

\begin{tabular}{|c|c|}
\hline $\begin{array}{c}\Delta \mathrm{K}_{\text {eff }} \\
\mathrm{Mpa} \sqrt{\mathrm{m}}\end{array}$ & $\begin{array}{c}\text { da/dN } \\
\text { m/cycle }\end{array}$ \\
\hline 3.75 & $3.0 \mathrm{E}-10$ \\
5.30 & $2.0 \mathrm{E}-09$ \\
7.30 & $7.0 \mathrm{E}-09$ \\
15.00 & $4.5 \mathrm{E}-08$ \\
50.00 & $5.5 \mathrm{E}-07$ \\
120.00 & $3.0 \mathrm{E}-05$ \\
\hline
\end{tabular}

In Table 4.2, the values of $\Delta \mathrm{K}_{\text {eff }}$ and $\mathrm{da} / \mathrm{dN}$ are linearly interpolated between two consecutive data points in the logarithmic scale while those beyond the maximum and minimum are extrapolated. In the simulation, the fatigue life is first predicted using 
the strain-life approach. This fatigue life is assumed to be identical to that obtained via the crack closure model, and is used to identify the initial crack size. The damage, in this case, is defined as the normalized crack length with respect to its critical length to failure (i.e., $\mathrm{D}=\mathrm{a} / \mathrm{a}^{*}$ ) where the critical crack length $\mathrm{a}^{*}$ is obtained from fracture toughness of the material. The material and fatigue properties of AISI 4340 steel are given in Table 4.3 [Boller and Seeger (1987)].

Table 4.3 Material Properties of AISI 4340

\begin{tabular}{|c|c|}
\hline Young's Modulus $(\mathrm{E})$ & $193500 \mathrm{~N} / \mathrm{mm}^{2}$ \\
Yield Strength, Monotonic $\left(\sigma_{\mathrm{y}}\right)$ & $1374 \mathrm{~N} / \mathrm{mm}^{2}$ \\
Yield Strength, Cyclic $\left(\sigma_{y^{\prime}}\right)$ & $905 \mathrm{~N} / \mathrm{mm}^{2}$ \\
Cyclic-Strength Coefficient $\left(\mathrm{K}^{\prime}\right)$ & $1890 \mathrm{~N} / \mathrm{mm}^{2}$ \\
Cyclic-Strength Hardening Exponent $\left(\mathrm{n}^{\prime}\right)$ & 0.118 \\
Fatigue-Strength Coefficient $\left(\sigma_{\mathrm{f}}^{\prime}\right)$ & $1880 \mathrm{~N} / \mathrm{mm}^{2}$ \\
Fatigue-Strength Exponent $(\mathrm{b})$ & -0.086 \\
Fatigue-Ductility Coefficient $\left(\varepsilon_{\mathrm{f}}^{\prime}\right)$ & 0.706 \\
Fatigue-Ductility Exponent $(c)$ & -0.662 \\
\hline
\end{tabular}

The results of the nonlinear damage $D$ versus the linear damage $D_{\ell}$ which is essentially the cycle ratio are used to compute $\gamma$ from eq. (4.20). Figure 4.15 shows the relationship between $\gamma$ and $D$ for different values of the (constant) stress amplitude $\sigma_{a}$ as a series of curves in the logarithmic scale. If these curves are generated to be closely spaced, the values between two curves can be obtained via linear interpolation without any significant error. Once the $\gamma$-parameters are computed as a function of two independent variables $\sigma_{a}$ and $D$, the nonlinear damage model described above can be readily used to simulate the nonlinear damage behavior. The nonlinear damage accumulations corresponding to different stress amplitudes are plotted in Figure 4.16.

It is seen in Figure 4.15 that $\gamma$-parameter is strongly dependent on the current damage level in contrast to the conventional damage curve approach where $\gamma_{\text {is }}$ assumed to be constant. At the initial stage of fatigue life, $\gamma$-parameters are only modestly larger than one so that it is more realistic for implementation in computer simulation. In general, the values of $\gamma$ are larger for smaller stress amplitudes. This indicates higher degrees of nonlinearity in high cycle fatigue as can be seen from the damage curves in Figure 4.16. The accumulated damage is much smaller in the early stage of fatigue life compared to the linear damage accumulation and accelerates much faster when the damage is close to failure. This implies a significant improvement in fatigue life prediction based on the nonlinear damage rule if the $\gamma$-parameters are properly selected from the experimental observations or on the basis of analytical results. 


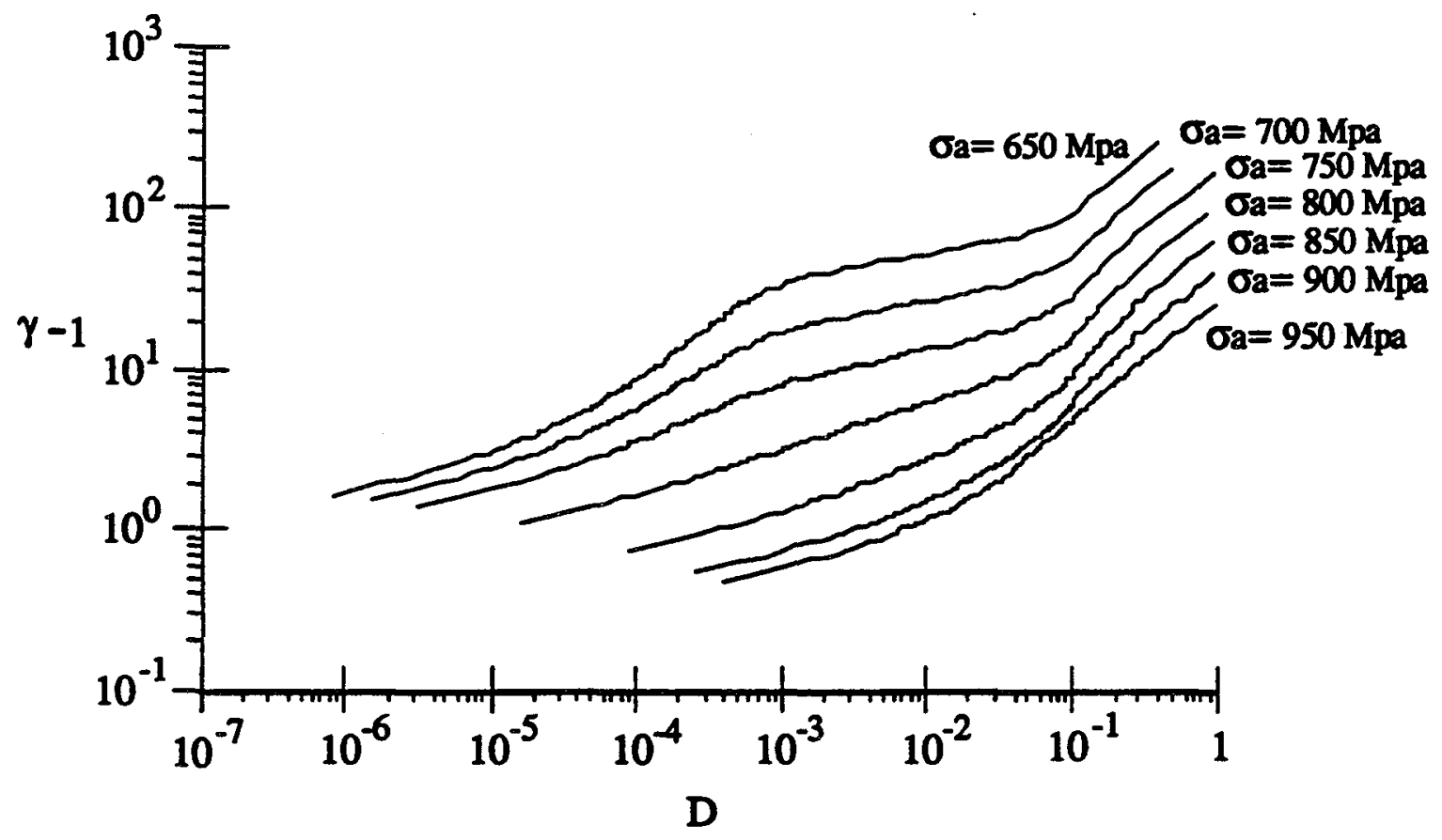

Figure $4.15 \gamma_{\text {versus }} D$ at Different Amplitudes

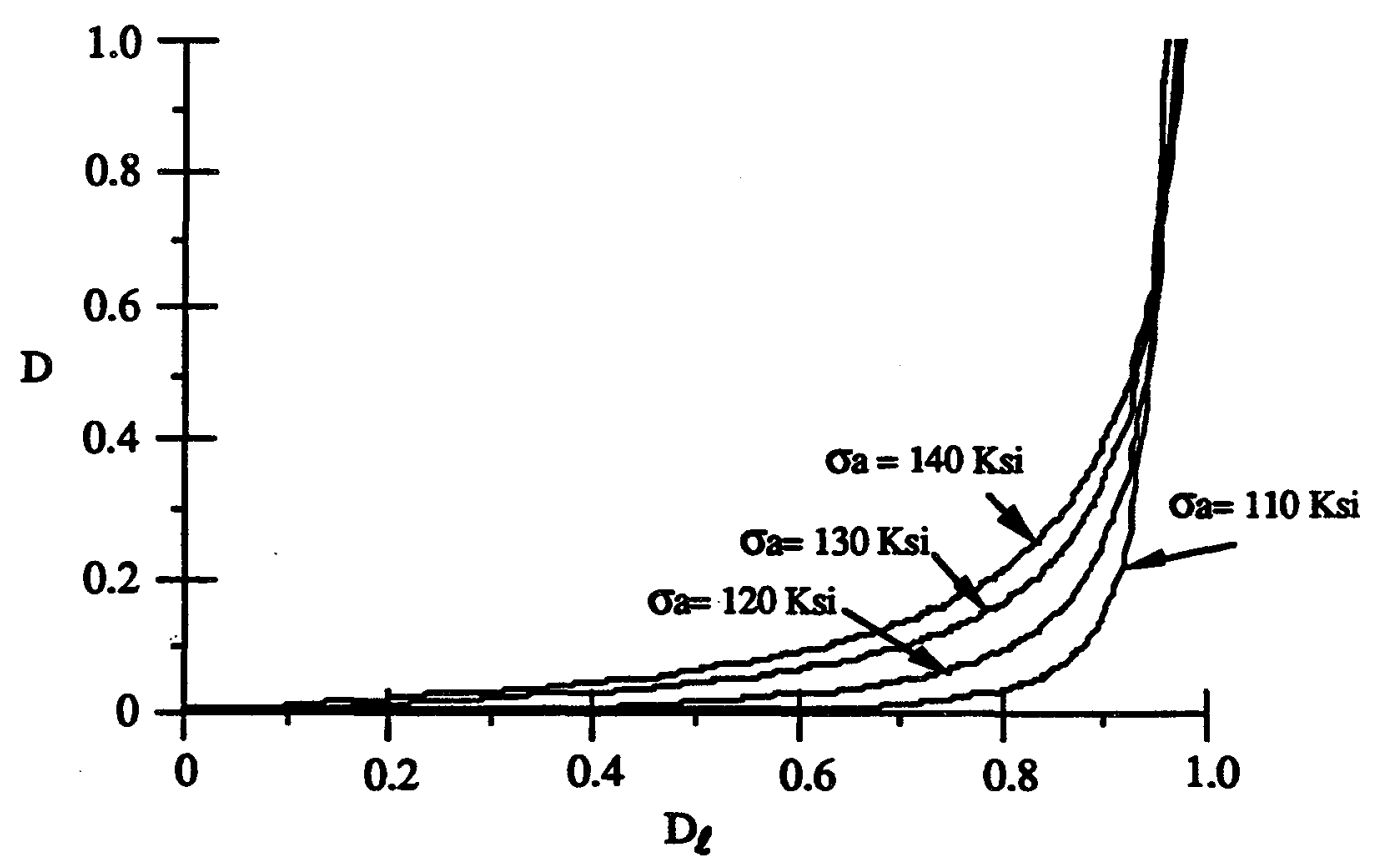

Figure 4.16 Nonlinear Damage Curves at Different Stress Amplitudes 


\subsection{Continuous-Time Damage Modeling of Fatigue Crack Growth}

The concept of a continuous-time damage model, developed in Section 4.2 using the strain-life approach, can be extended to formulate a continuous-time model for crack propagation. In the most commonly used fatigue crack growth models, the growth rate of the crack length, a, relative to the number of cycles, $\mathrm{N}$, is directly dependent on the stress intensity factor range, $\Delta \mathrm{K}$. Most of these models can be expressed in a mathematical form as:

$$
\frac{d a}{d N}=f_{1}(\Delta K)=f(\Delta \sigma, a)
$$

where $\Delta \mathrm{K}$ is a function of both stress amplitude and crack length. In this report, the crack closure model is adopted and therefore $\Delta \mathrm{K}$ is replaced by the effective stress intensity factor range $\Delta \mathrm{K}_{\text {eff }}$ and $\Delta \sigma=\sigma-\sigma_{0}$, where $\sigma_{0}$ is the crack opening stress. To be consistent with the strain-life approach, damage is assumed to be identically equal to 1 when the crack length reaches the critical value, $a^{*}$, which can be estimated from the fracture toughness of the component. The damage is assumed to occur only if the instantaneous stress is increasing and the crack opening stress, $\sigma_{0}$, is exceeded [Newman, 1981]. This implies that the damage rate is equal to zero during unloading. The damage increment $\Delta \mathrm{D}$ during the rising reversal can be expressed as a function of $\Delta \sigma$ and $\mathrm{D}:$

$$
\Delta \mathrm{D}=\frac{\mathrm{dD}}{\mathrm{dN}}=\frac{1}{\mathrm{a}^{*}} \times \frac{\mathrm{da}}{\mathrm{dN}}=\frac{1}{\mathrm{a}^{*}} \mathrm{f}\left(\Delta \sigma, \mathrm{a}^{*} \mathrm{D}\right) \text { if } \sigma>\sigma_{0}
$$

where the fatigue damage is defined as $\mathrm{D}=\mathrm{a} / \mathrm{a}^{*}$ in the absence of a more precise definition of damage. Since the damage increment is relatively small, the total damage $D$ is assumed to remain constant during a reversal. The damage rate can be obtained by differentiating eq. (4.29) with respect to time.

$$
\frac{d D}{d t}=\frac{1}{a^{*}} \times \frac{d f\left(\Delta \sigma, a^{*} D\right)}{d \sigma} \times \frac{d \sigma}{d t}
$$

A simple example based on the Paris model [Paris and Erdogan (1963)] is given below.

Let $\left\{t_{k}\right.$ \} be the sequence of time instants when damage values are to be estimated, and $\left\{\sigma\left(t_{k}\right)\right\}$ be the corresponding sequence of stresses. Then, the Paris model can be modified to yield the time derivative of damage as:

$$
\begin{array}{ll}
\frac{d D}{d t}=\frac{C}{a^{*}} n\left(\sigma-\sigma_{0}\right)^{n-1}\left(\sqrt{\pi a^{*} D} F\left(a^{*} D, w\right)\right)^{n} \frac{d \sigma}{d t} & \text { if } \sigma\left(t_{k}\right)>\sigma\left(t_{k-1}\right) \text { and } \sigma\left(t_{k}\right)>\sigma_{0} \\
\frac{d D}{d t}=0 & \text { otherwise }
\end{array}
$$


where $F$ is the correction factor depending on the geometry and $w$ is the width of the specimen. The application of eq. (4.31) requires estimation of the crack opening stress, $\sigma_{0}$, which can be calculated from the crack closure model [Newman (1981)] based on the load history. Following a simplified method [Newman (1984)] for computing $\sigma_{0}$, the damage increment between two consecutive instants $t_{k-1}$ and $t_{k}$ can be obtained by integrating eq. (4.31) as follows:

$$
\begin{aligned}
& D\left(t_{k}\right)-D\left(t_{k-1}\right)=\int_{t_{k-1}}^{t_{k}}\left(\frac{d D}{d t}\right) d t \\
& =\frac{C}{a^{*}}\left[\left(\sigma\left(t_{k}\right)-\sigma_{0}\right)^{n}-\left(\operatorname{Max}\left(\sigma\left(t_{k-1}\right), \sigma_{0}\right)-\sigma_{0}\right)^{n}\right] \times\left(\sqrt{\pi a^{*} D\left(t_{k-1}\right)} F\left(a^{*} D\left(t_{k-1}\right), w\right)\right)^{n} \\
& D\left(t_{k}\right)=D\left(t_{k-1}\right) \\
& \text { if } \sigma\left(t_{k}\right)>\sigma\left(t_{k-1}\right) \text { and } \sigma\left(t_{k}\right)>\sigma_{0} \\
& \text { otherwise. }
\end{aligned}
$$




\section{CHAPTER 5}

\section{SIMULATION RESULTS AND DISCUSSIONS}

The damage mitigation concept, described in Chapter 2 , has been verified by simulation experiments for open loop control of a reusable rocket propulsion engine such as one described in [Sutton (1992); Duyar et al. (1991)]. The plant model under control is a simplified representation of the dynamic characteristics of a bipropellant rocket engine as shown schematically in Figure 5.1. The preburner serves as a gas generator for driving the liquid hydrogen $\left(\mathrm{LH}_{2}\right)$-fuel turbopump. In this model, oxidant is separately supplied to the preburner and the main combustor chamber. Standard lumped parameter methods have been used to model the nonlinear plant dynamics in

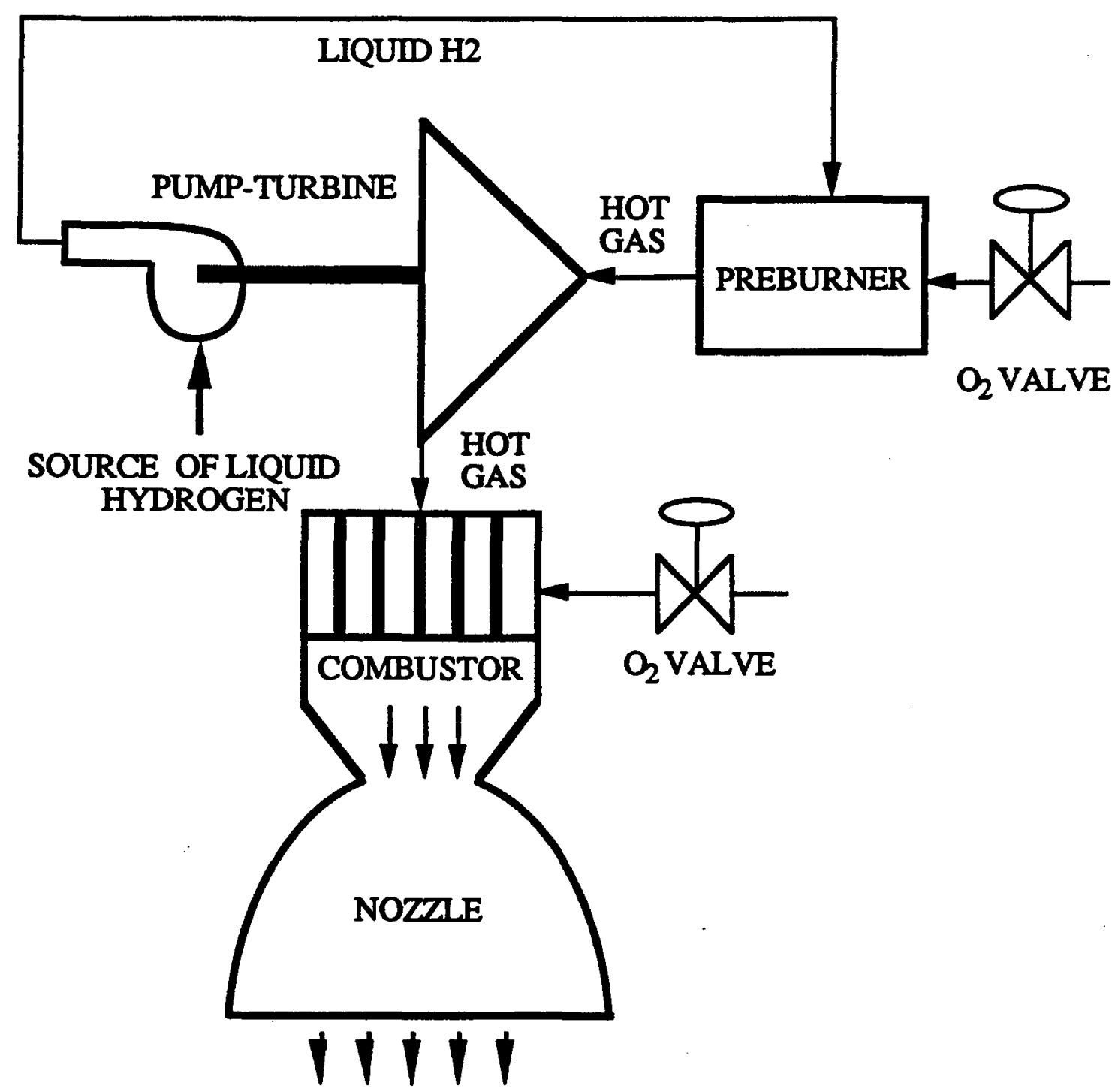

Figure 5.1 Schematic Diagram of a Bipropellant Rocket Engine 
the state-space form where the plant state vector consists of turbine shaft speed, pump $\left(\mathrm{LH}_{2}\right.$-fuel) mass flow rate, preburner gas pressure, preburner gas density, combustor gas pressure, combustor gas density, and the two flow rates of oxidant into the preburner and the main combustor, respectively. The critical plant output variables are combustor gas pressure and the oxidant/ fuel $\left(\mathrm{O}_{2} / \mathrm{H}_{2}\right)$ ratio, and the control inputs are the areas of the two oxidant valves. The governing equations for the lumped parameter model of the plant are given in Appendix C. The structural model, as delineated in Appendix D, calculates the cyclic stresses at the root of a typical turbine blade that is presumed to be a critical component. The blade is represented by a three-node beam model with six degrees of freedom at each node while the first node is kept fixed. The load on the blade is assumed to consist of two components. The first component is due to the (time-dependent) drive torque which is derived as an output of the plant model. The second component is a dynamic term which represents the oscillatory load on the blade as it passes each stator. It is the second component that causes high cycle fatigue at the root of the blade while the first component is largely responsible for the mean stress. The fatigue damage model formulated in Chapter 4 was used to generate the results in Figures 5.2 to 5.14.

\subsection{Simulation of the Bipropellant Rocket Engine under Open-Loop Control}

The simulation experiments serve to evaluate the plant dynamic performance and damage of the critical component when the oxidant valves are manipulated to vary the engine thrust following the open-loop control policy developed in Chapter 3. To demonstrate the broad concepts of fatigue damage mitigation, the nominal plant model was used in the simulation experiments with exact initial conditions and no disturbances and noise. However, if these conditions are not met, additional feedback control will be necessary because the open-loop control alone would be inadequate for plant operations as discussed in Appendix A. Following the structure in eq. (3.3), the cost functional $\mathrm{J}$ for nonlinear programming was selected to generate the open loop control policy as:

$$
J=\sum_{\mathbf{k}=0}^{N-1}\left[\tilde{\mathbf{x}}_{\mathbf{k}}{ }^{\mathrm{T}} \mathbf{Q} \tilde{\mathbf{x}}_{\mathbf{k}}+\dot{\mathbf{v}}_{\mathbf{k}}^{\mathrm{T}} \mathbf{S} \dot{\mathbf{v}}_{\mathbf{k}}+\tilde{\mathbf{u}}_{\mathbf{k}}{ }^{\mathrm{T}} \mathbf{R} \tilde{\mathbf{u}}_{\mathbf{k}}+\mathrm{W}\left(\mathrm{O}_{2} / \mathrm{H}_{2}\right)_{\mathbf{k}}{ }^{2}\right]
$$

where the deviations, $\tilde{\mathbf{x}}_{\mathbf{k}}$ and $\tilde{\mathbf{u}}_{\mathbf{k}}$, in the plant state vector and the control input vector are as defined in eq. (3.3); and the diagonal matrices $\mathbf{Q}, \mathbf{S}, \mathbf{R}$ and the scalar $\mathbf{W}$ serve as relative weights of the individual variables. Since the rocket engine performance is very sensitive to the oxidant/fuel $\left(\mathrm{O}_{2} / \mathrm{H}_{2}\right)$ ratio, it was brought into the cost functional in eq. (5.1) to prevent any large deviations from the desired value through the transients. If the main combustion chamber is selected as one of the critical components for damage mitigation, then the gas temperature has to be controlled within a small bound. In that case, the cost functional in eq. (5.1) need not be explicitly dependent on the $\mathrm{O}_{2} / \mathrm{H}_{2}$ ratio which is directly related to the main chamber temperature in the range of normal operations. Simulation results were obtained under the following conditions:

Chamber pressure weight $Q_{55}=12$; and all other weights $Q_{i i}=1, i \neq 5$; Control input weight $\mathbf{R}=\mathbf{I}$ where $\mathrm{I}$ is the identity matrix;

Damage Rate Weight $S_{i i}=0, i=1, \cdots, N ;$ and $\mathrm{O}_{2} / \mathrm{H}_{2}$ ratio weight $\mathrm{W}=10$. 
The rocket engine model is initiated from an initial equilibrium condition at 2700 psi chamber pressure and $\mathrm{O}_{2} / \mathrm{H}_{2}$ ratio of 6.02 . From this condition the optimization procedure steers the plant to a new equilibrium position at $3000 \mathrm{psi}$ and the same $\mathrm{O}_{2} / \mathrm{H}_{2}$ ratio of 6.02 in 50 milliseconds. The control commands to the two oxidant valves, are updated at every one millisecond. That is, $\mathrm{N}$ is equal to 50 in eq. (5.1). The performance cost to be minimized is based on the deviations from the final equilibrium condition at $3000 \mathrm{psi}$. The results of simulation experiments for the two following conditions are presented as series of curves in Figures 5.2 to 5.14:

Simulation Condition 1: Initial damage is set to $D_{0}=0.01$ for the unconstrained case and two constrained cases in which the damage rate constraints are listed in Table 5.1.

Simulation Condition 2: To examine the effects of initial damage on nonlinear damage accumulation, simulation results are generated under three different initial values of the accumulated damage, namely, $\mathrm{D}_{0}=0.001,0.01$ and 0.1 while the damage rate constraint is set identical to that of the constrained case 1 in Table 5.1.

For both conditions 1 and 2, the accumulated damage $(\varphi)$ was not constrained.

Table 5.1 The Constraints for the Two Cases under Simulation Condition 1

Case 1

Time

$0 \mathrm{~ms}$ to $2 \mathrm{~ms}$

$2 \mathrm{~ms}$ to $3 \mathrm{~ms}$

$3 \mathrm{~ms}$ to $50 \mathrm{~ms}$
Damage rate constraint ( $\beta$ )

$1.0 \times 10^{-6} \mathrm{sec}^{-1}$

$2.5 \times 10^{-6} \mathrm{sec}^{-1}$

$5.0 \times 10^{-6} \mathrm{sec}^{-1}$
Case 2

Damage rate constraint ( $\beta$ )

$0.2 \times 10^{-6} \mathrm{sec}^{-1}$

$0.5 \times 10^{-6} \mathrm{sec}^{-1}$

$1.0 \times 10^{-6} \mathrm{sec}^{-1}$

\subsection{Results and Discussions}

The transient responses in Figures 5.2 to 5.14 examine the various engine variables under the above two simulation conditions. Figures 5.2 and 5.3 show the transient responses of the two oxygen valves resulting from the optimization over the time frame of 0 to $50 \mathrm{~ms}$ where the control action is updated at every millisecond. The corresponding changes in the oxidant flows are seen in Figures 5.4 and 5.5. Figure 5.6 also shows the transients of the fuel flow (i.e., liquid hydrogen). The responses of both control valves (and therefore the oxidant flows) become more restricted and sluggish as the damage rate constraint is made stronger. Similar effects are observed when the initial damage is increased. The rationale for this behavior is that, for a given stress amplitude, the damage rate increases with an increase in the initial damage. This dependence on the initial damage results from the $\gamma$-parameter in the nonlinear damage model as defined in eq. (4.19) in Chapter 4, and does not occur in the linear damage model where $\gamma$ is identically equal to 1 . It is important to note that $\gamma$ is greater than 1 in this study because the AISI 4340 steel used here is a high strength material. However, for ductile materials such as copper-base alloys used in the thrust chamber cooling

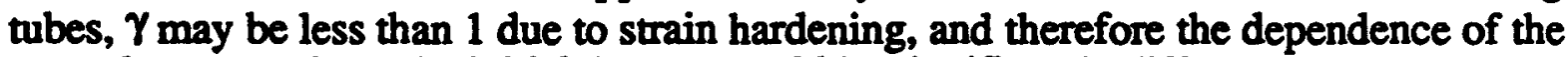
control commands on the initial damage could be significantly different. 
Figures 5.7 to 5.11 exhibit the effects of the varying oxygen inlet flow to the preburner and the main combustor on the engine dynamics. The resulting transients of the process variables, namely, $\mathrm{O}_{2} / \mathrm{H}_{2}$ ratio, and the pressure and temperature in the preburner and main combustion chamber, are shown for the two simulation conditions. As expected, for a given level of initial damage, both pressure and temperature dynamics tend to be slower as the constraint is made more severe. Similar effects are seen by increasing the initial value, $\mathrm{D}_{0}$, of the accumulated damage. The combustor pressure is seen to rise monotonically in all cases after a small dip at about $2.5 \mathrm{~ms}$ while the preburner pressure keeps on increasing. These plots are largely similar except for the transients from $1 \mathrm{~ms}$ to $10 \mathrm{~ms}$. Virtually all of fatigue damage accumulation in this transient operation takes place during this short interval as seen in Figure 5.14. Furthermore, the net excursion of the $\mathrm{O}_{2} / \mathrm{H}_{2}$ ratio is in the range of 6.0 to 6.5 in all cases for the up-thrust transient of the rocket engine. The 6.5 mixture ratio is about the limit that would be tolerated during a transient excursion. The overshoot in the mixture ratio is caused by a drop in the hydrogen flow as seen in Figures 5.6 and 5.7. At this point the turbopump demands more torque to increase its speed so that the pump pressure can be elevated to generate a higher value of hydrogen flow for the desired mixture ratio. This results in a peak overshoot in the mean stress as shown in Figure 5.12. This sharp increase in stress is the major cause of enhanced damage in the turbine blades.

Since the turbine blades are the critical components for damage analysis in this study, the pressure ratio across the turbine which directly influences the torque is very important. For a given preburner pressure, as shown in Figure 5.9, a reduction in the combustor pressure causes an increase in the turbine torque. It is the turbine torque and speed that set the stress and fatigue damage on the turbine blades. Therefore, the dip in the combustor pressure at about $2.5 \mathrm{~ms}$ is largely responsible for the peak mean stress displayed in Figure 5.12. Both the mean stress and stress amplitude are the basic inputs to the damage model.

The graphs in Figures 5.12 to 5.14 compare the damage rate and the accumulated damage for the two simulation conditions along with the transient responses of the mean stress. For the unconstrained case, the peak stress causes the largest overshoot in the damage rate which is plotted on a logarithmic scale. In contrast, for the initial damage of 0.001 , the damage rate is within the limit of the constraint even though the peak of mean stress is the largest. This phenomenon is a result of a relatively small slope of the damage curve at the initial stages of the fatigue life. The accumulated damage, plotted on a linear scale, is seen to be significantly influenced by the constraints and also by the initial damage. This suggests that, for reusable rocket engines, the constraints need to be appropriately specified based on the knowledge of the initial damage. The damage rate is dependent on the sequences of control commands, and the oxidant flows into the preburner and combustor are changed in response. Therefore, if the initial damage cannot be accurately assessed, then it might be safe to generate the control command sequences on the assumption of a conservative, i.e., larger, value of the initial damage at the expense of the engine performance.

The important observation in these simulation experiments is the substantial reduction in the accumulated damage, thereby extending the service life of the turbopump. The accumulated damage in the unconstrained case is seen to be about four to 
twelve times that of the constrained case. This is a clear message that the consideration of damage in the control of transients to which a rocket engine is exposed can have a considerable impact on the life of critical components (in this case, the turbine blades). It is noted that there is practically no penalty in the response times of chamber pressure, i.e., the engine thrust, between the unconstrained and the constrained cases. If one is willing to pay a small price in response time, much larger gains on damage accumulation can be achieved.

Figures 5.2 to 5.14 exemplify the effects of upthrust transients during a short period of $50 \mathrm{~ms}$. Complete operations of a rocket engine during a single flight include many such upthrust transients, and the steady-state operation may last for several hundreds of seconds. Although the damage rate during the steady state is much smaller than that during a transient operation, the total damage accumulation during the steady state may not be relatively insignificant. Therefore, during a flight of the (reusable) rocket engine, the cumulative effects of the transient and steady state operations need to be considered in the optimization procedure as discussed in Chapter 3 .

The simulation experiments, described above, only consider a single point of critical stress, namely, the turbine blades. In this case, the damage vector is onedimensional. Simultaneous control of damage at several other critical areas in the rocket engine, such as the nozzle lining, shall render the damage vector to be multidimensional. The optimization problem is then to generate a control sequence that will not only make a trade-off between the performance and damage but also strike a balance between potentially conflicting requirements of damage mitigation at the individual critical points. 

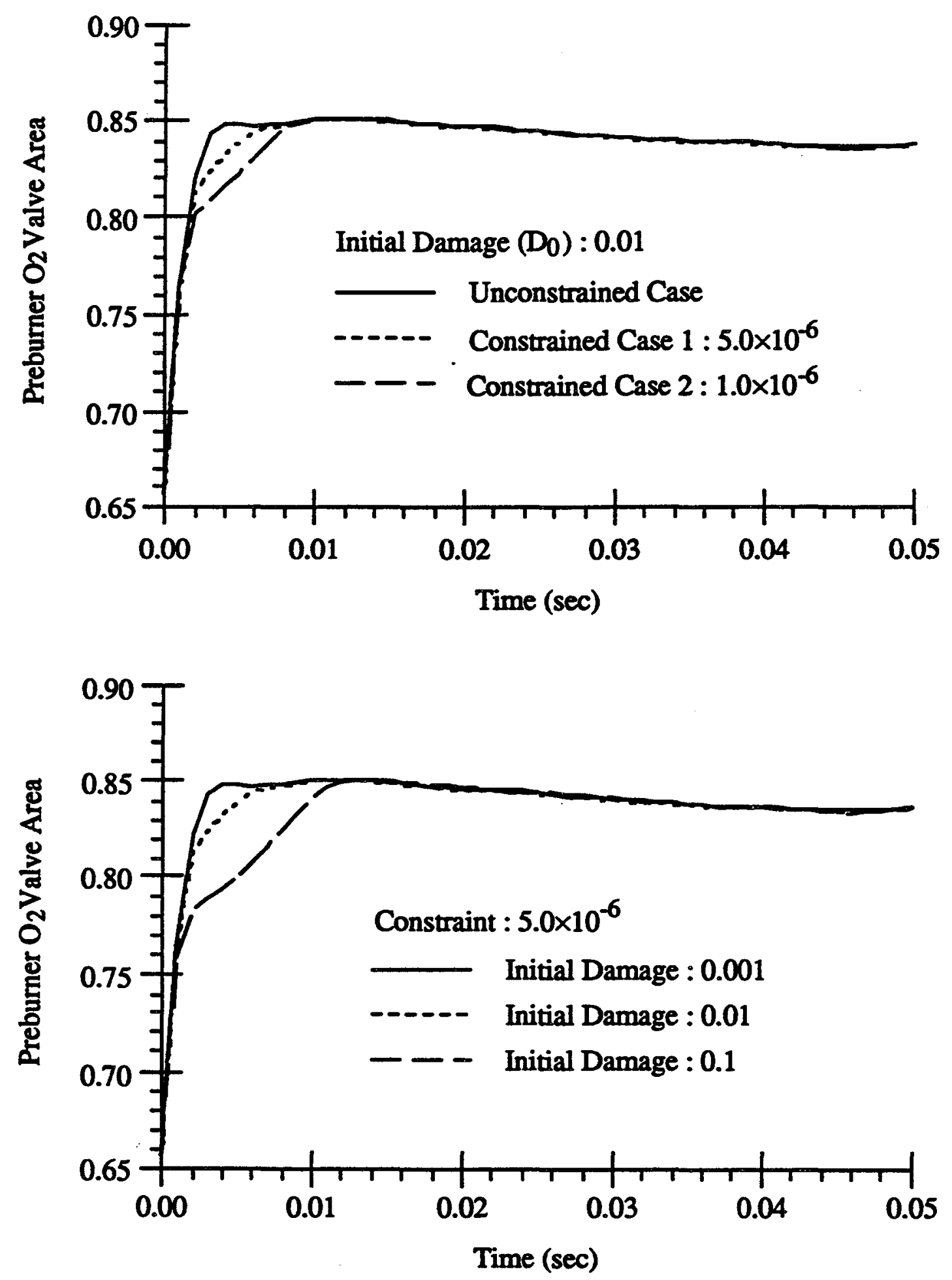

Figure 5.2 Transient Responses of Preburner $\mathrm{O}_{2}$ Valve Areas 

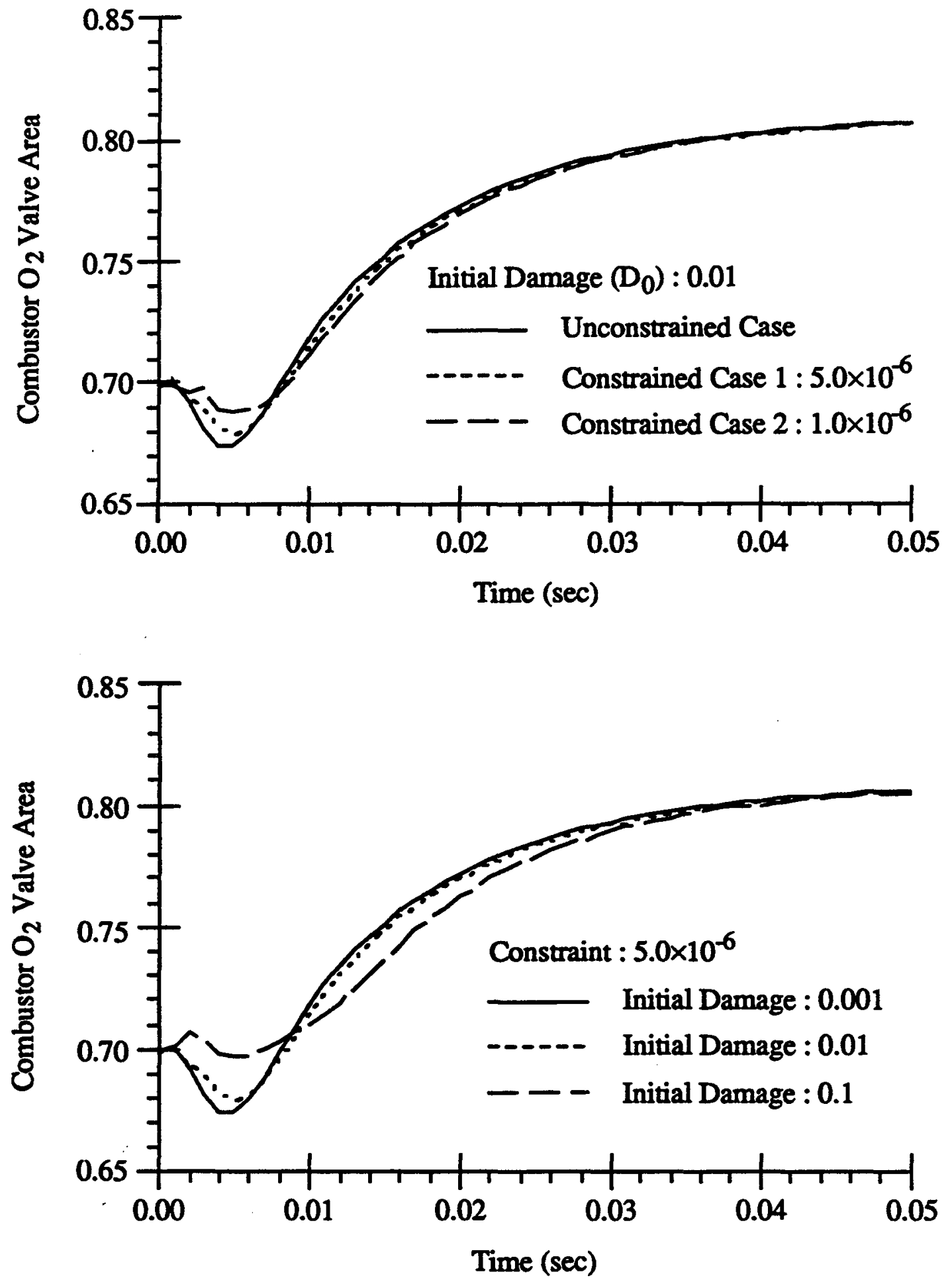

Figure 5.3 Transient Responses of Combustor $\mathrm{O}_{2}$ Valve Areas 

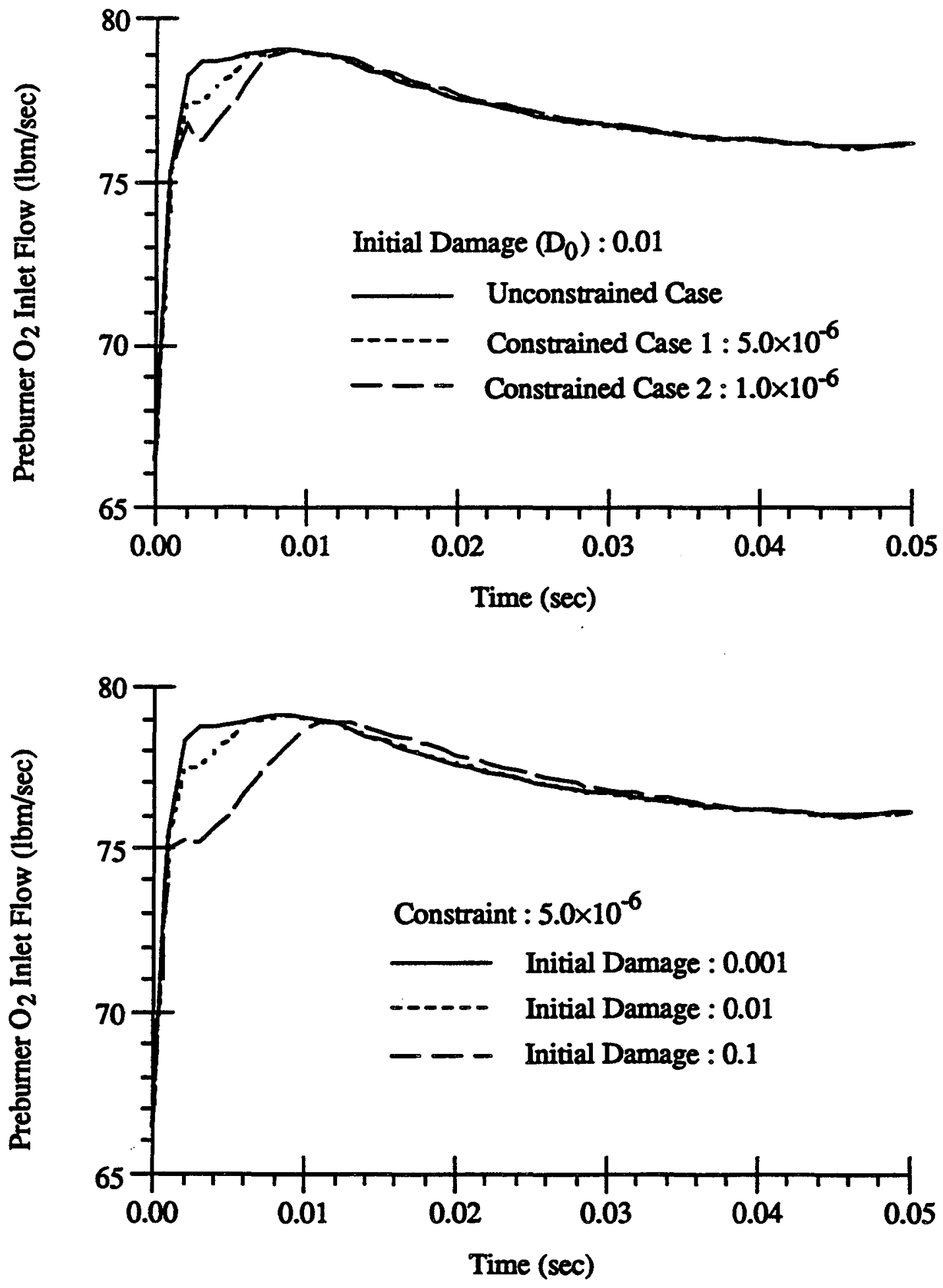

Figure 5.4 Transient Responses of Preburner $\mathrm{O}_{2}$ Inlet Flow 

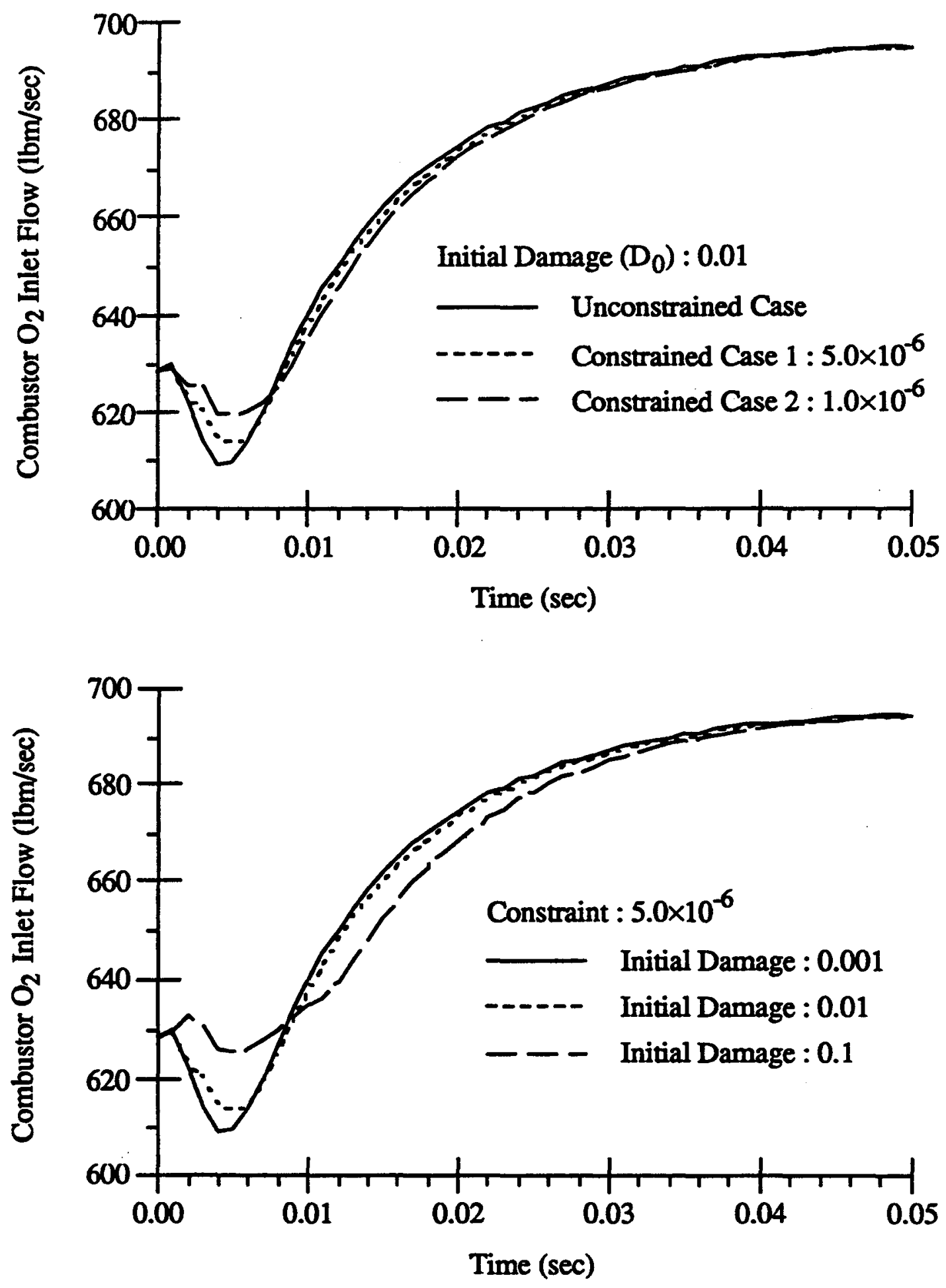

Figure 5.5 Transient Responses of Combustor $\mathrm{O}_{2}$ Inlet Flow 

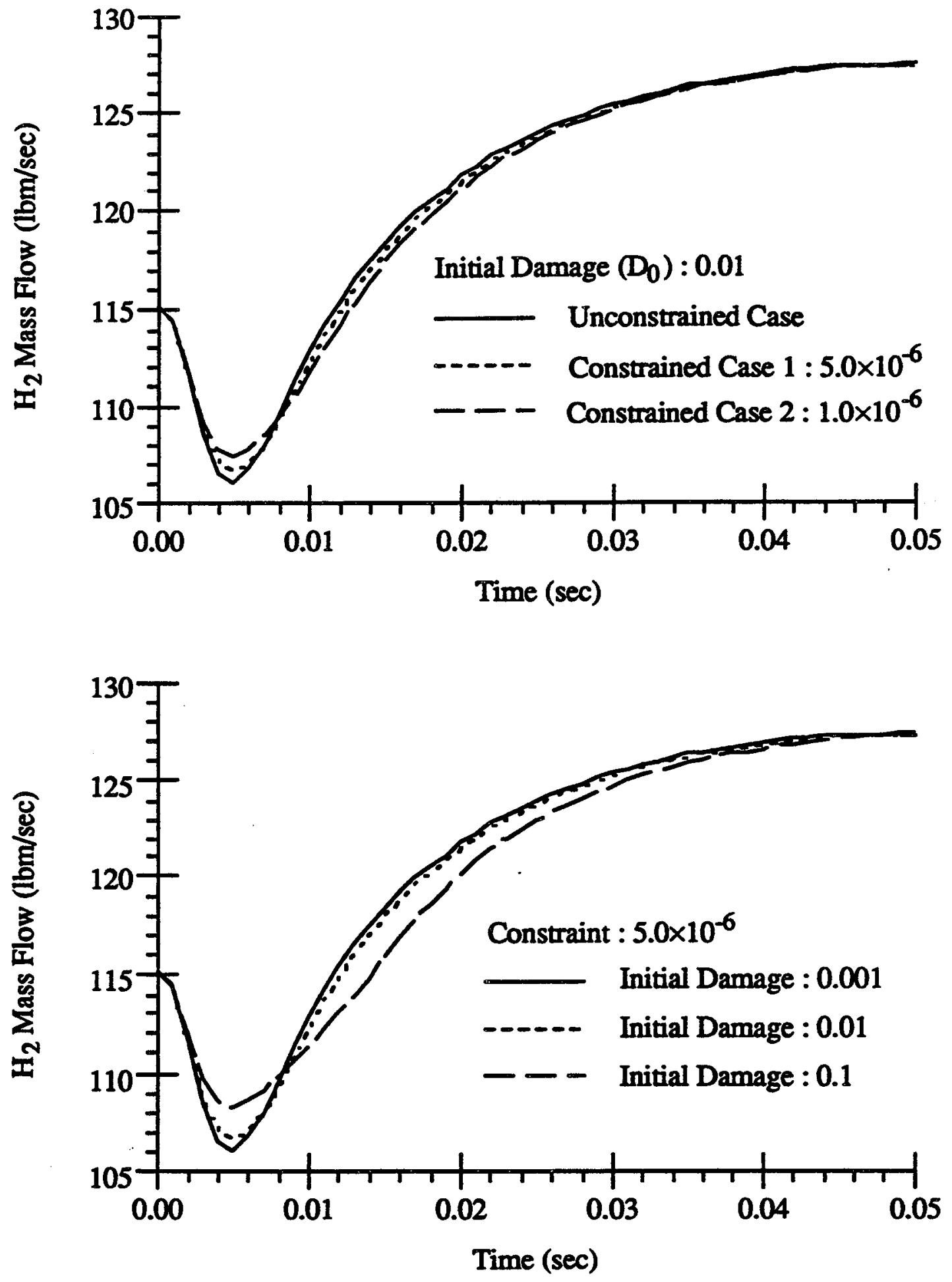

Figure 5.6 Transient Responses of $\mathrm{H}_{2}$ Mass Flow 

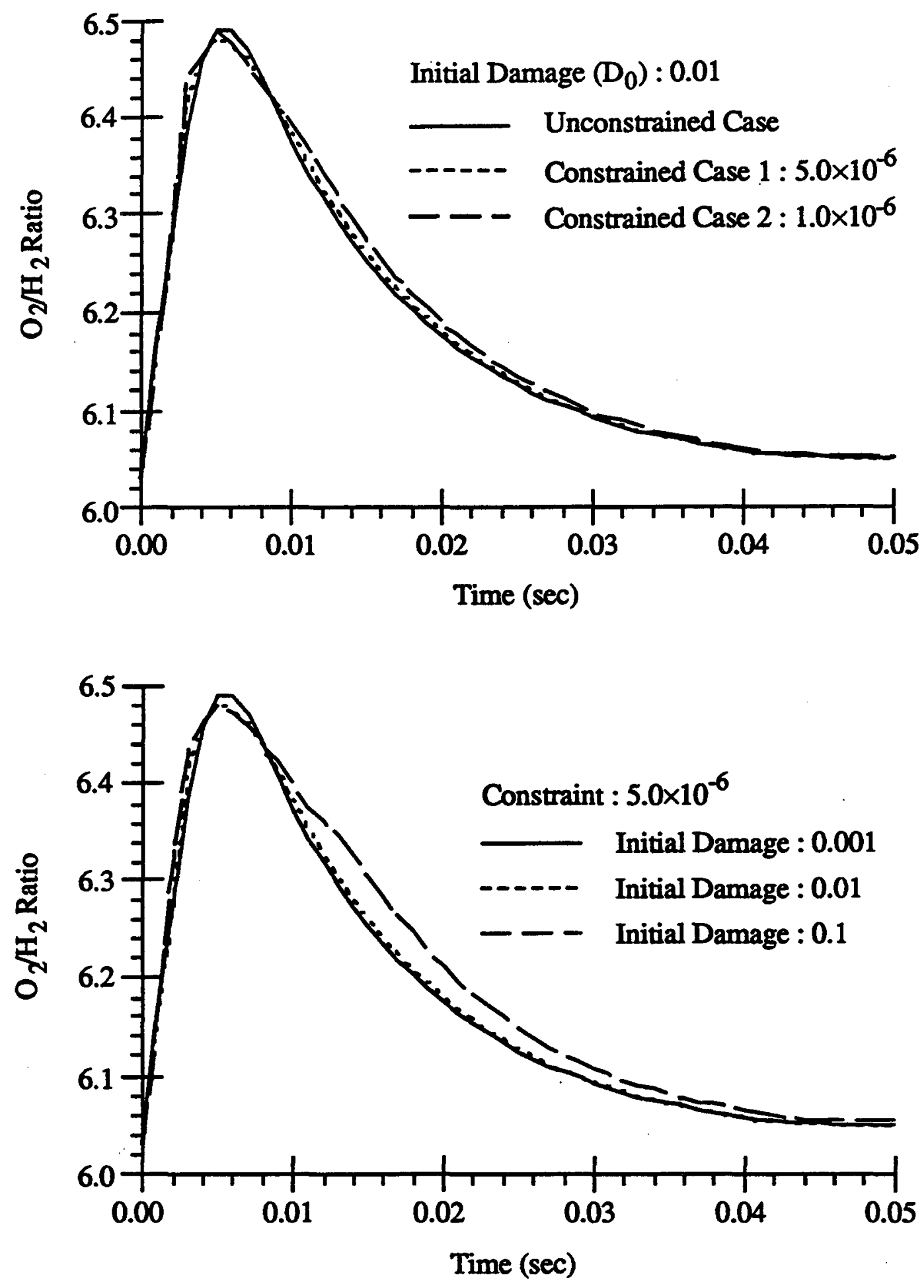

Figure 5.7 Transient Responses of $\mathrm{O}_{2} / \mathrm{H}_{2}$ Ratio 

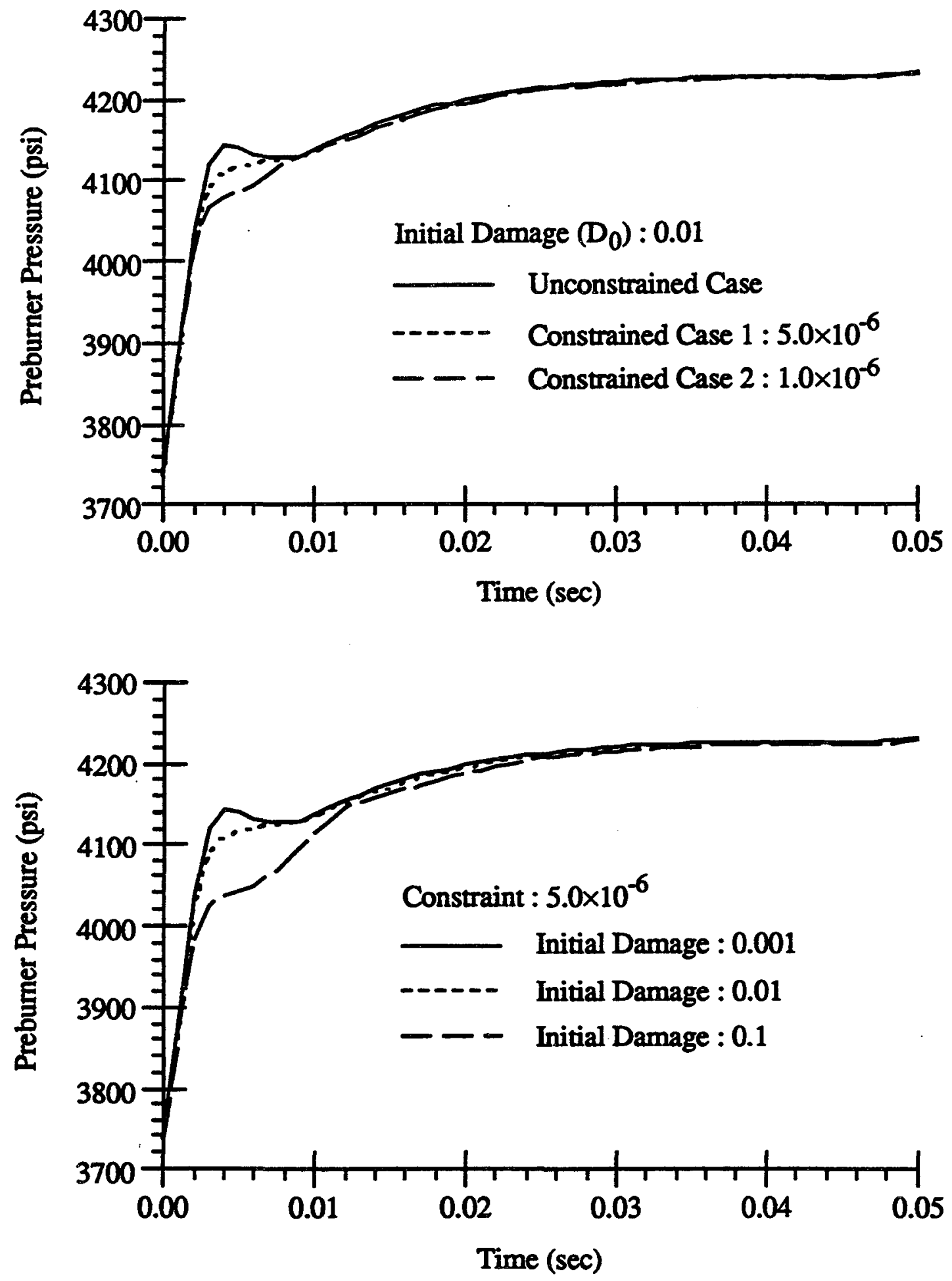

Figure 5.8 Transient Responses of Preburner Pressure 

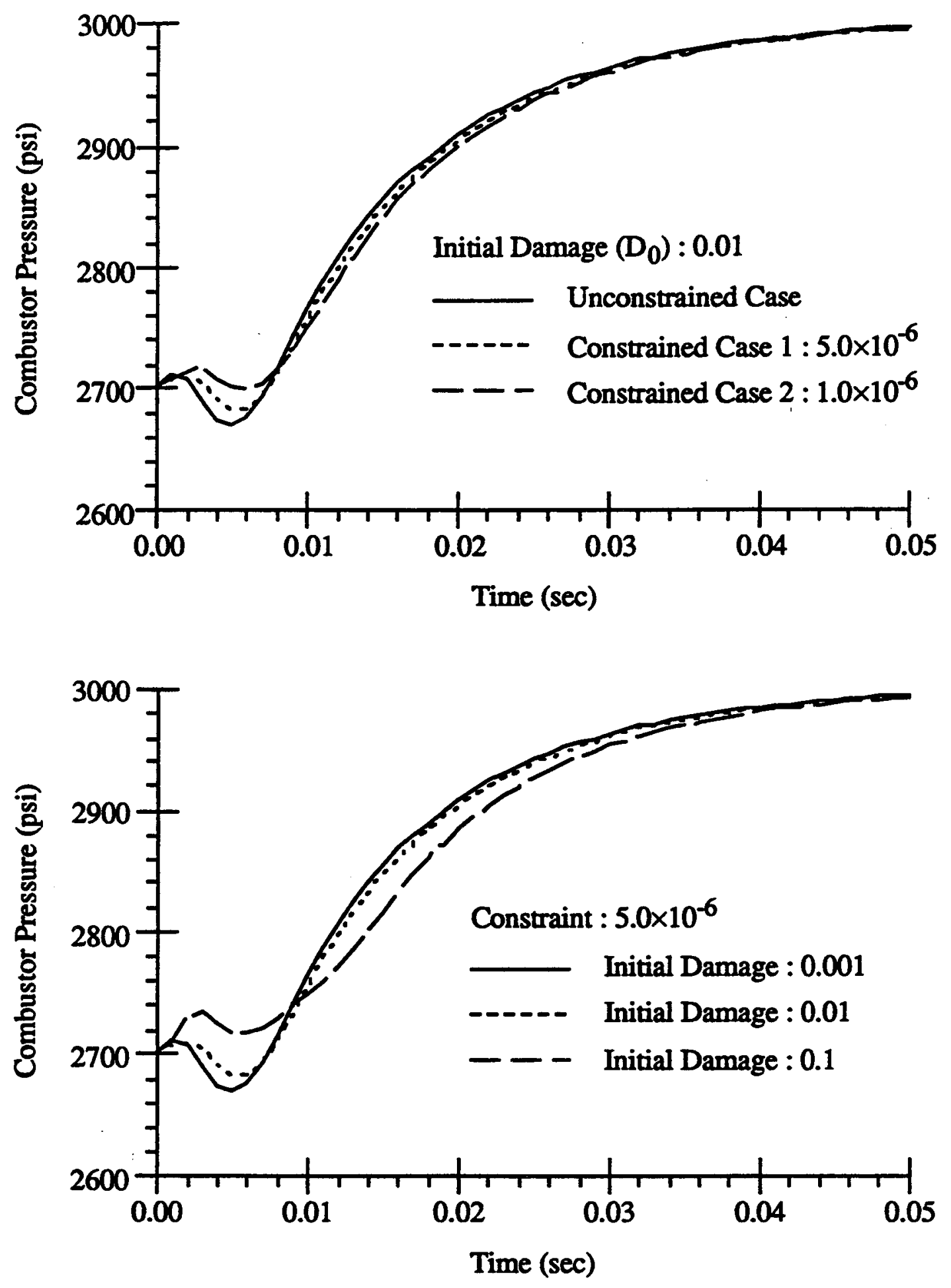

Figure 5.9 Transient Responses of Combustor Pressure 

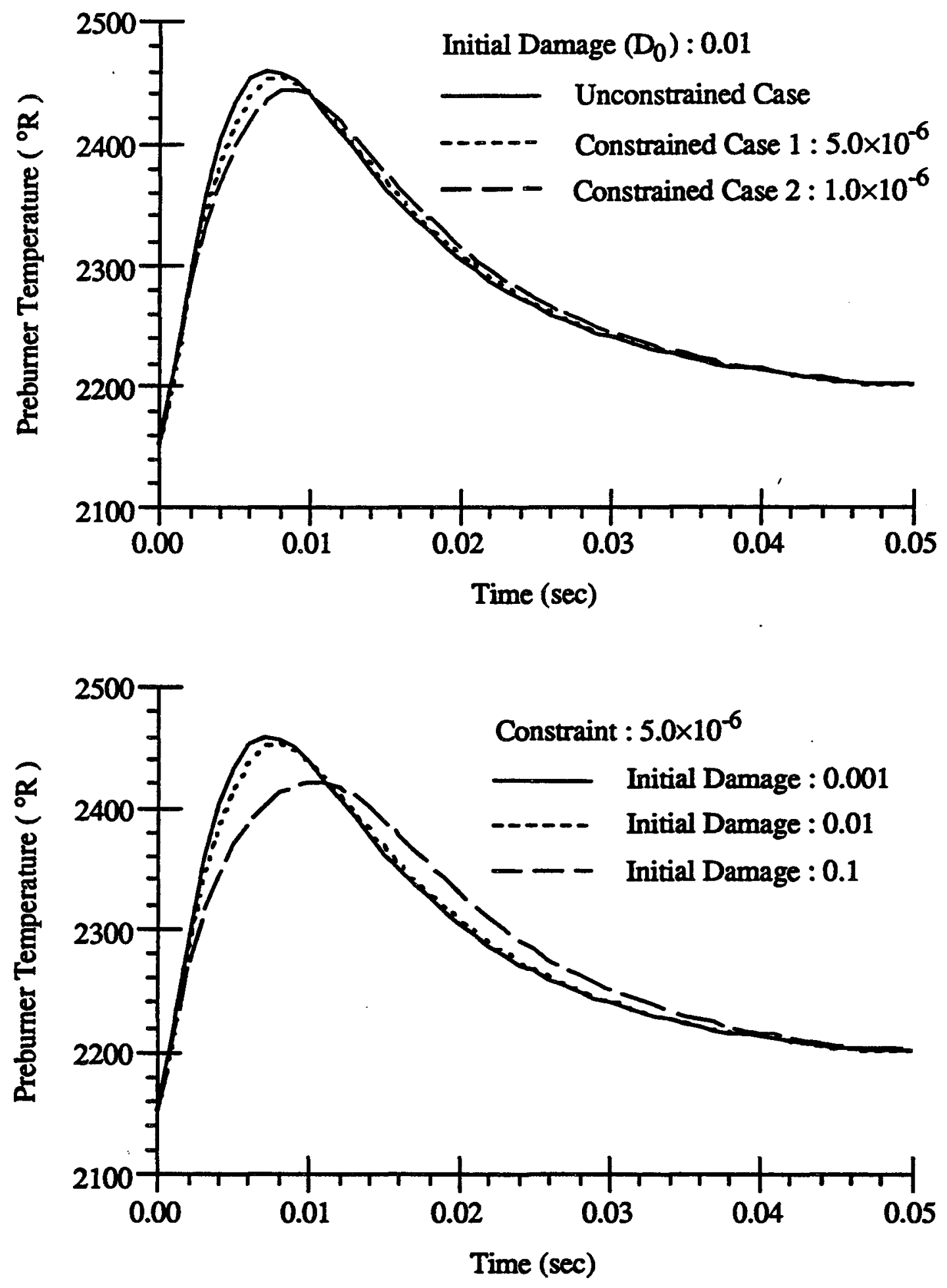

Figure 5.10 Transient Responses of Preburner Temperature 

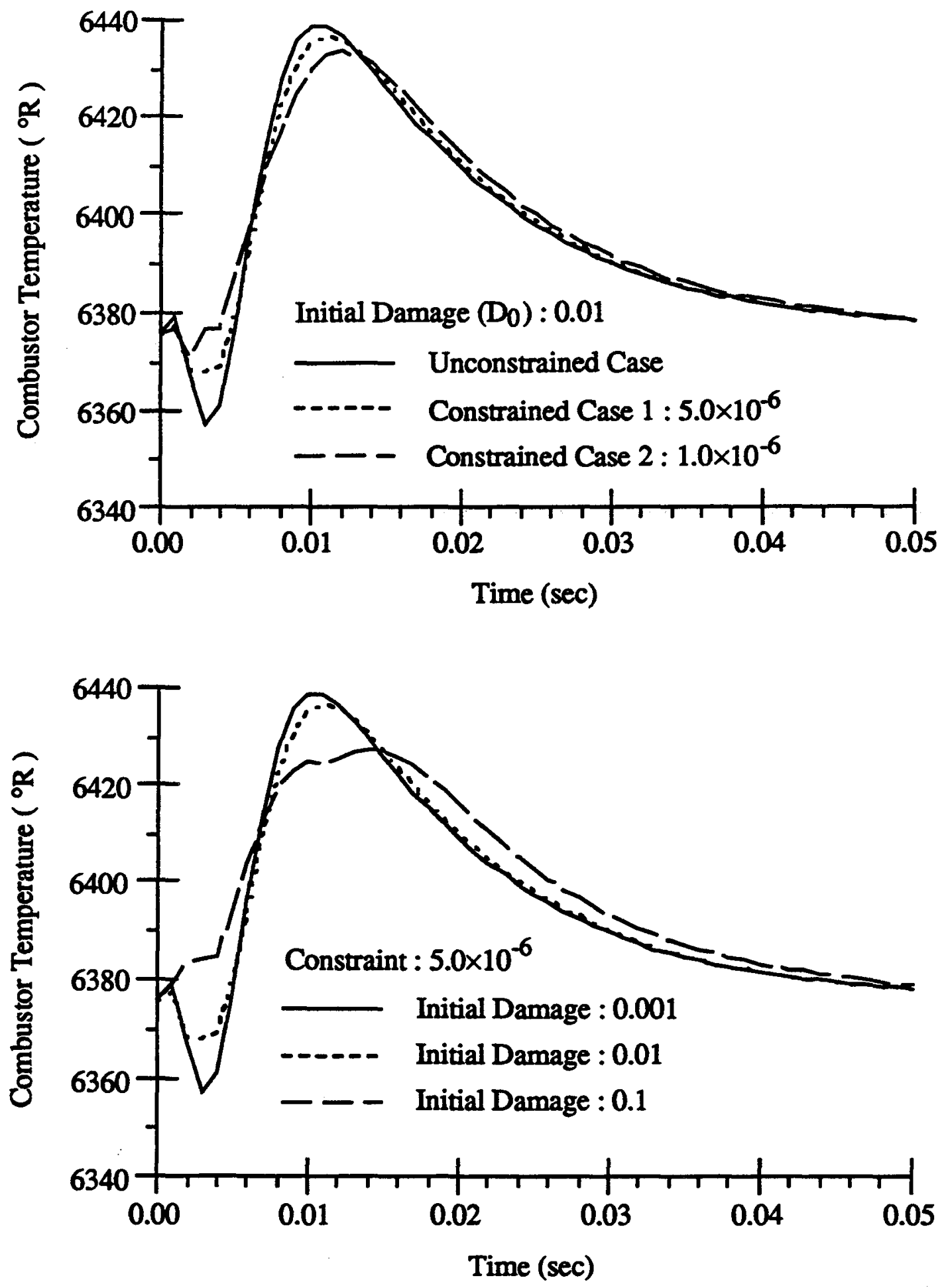

Figure 5.11 Transient Responses of Combustor Temperature 

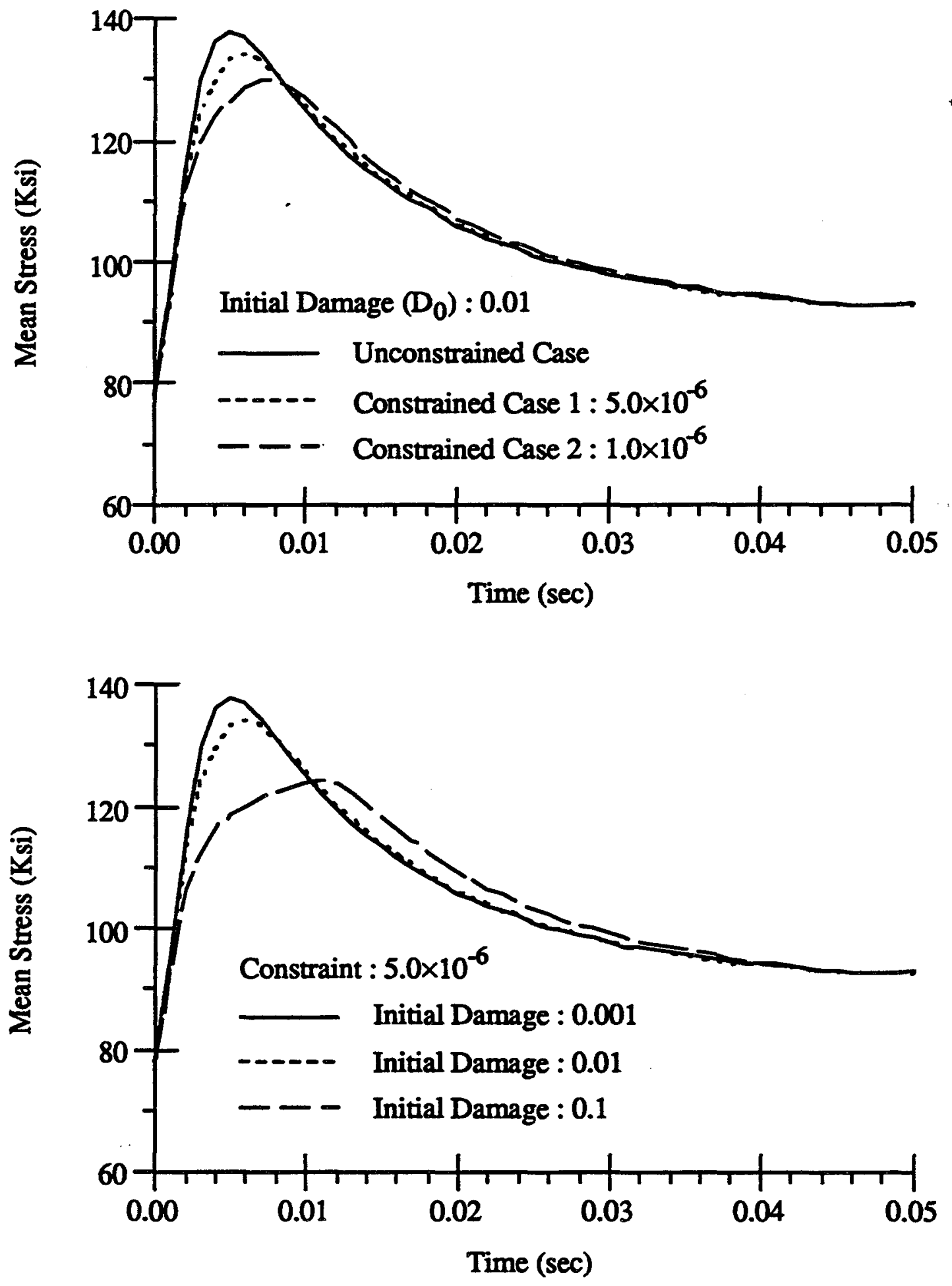

Figure 5.12 Transient Responses of Mean Stress 

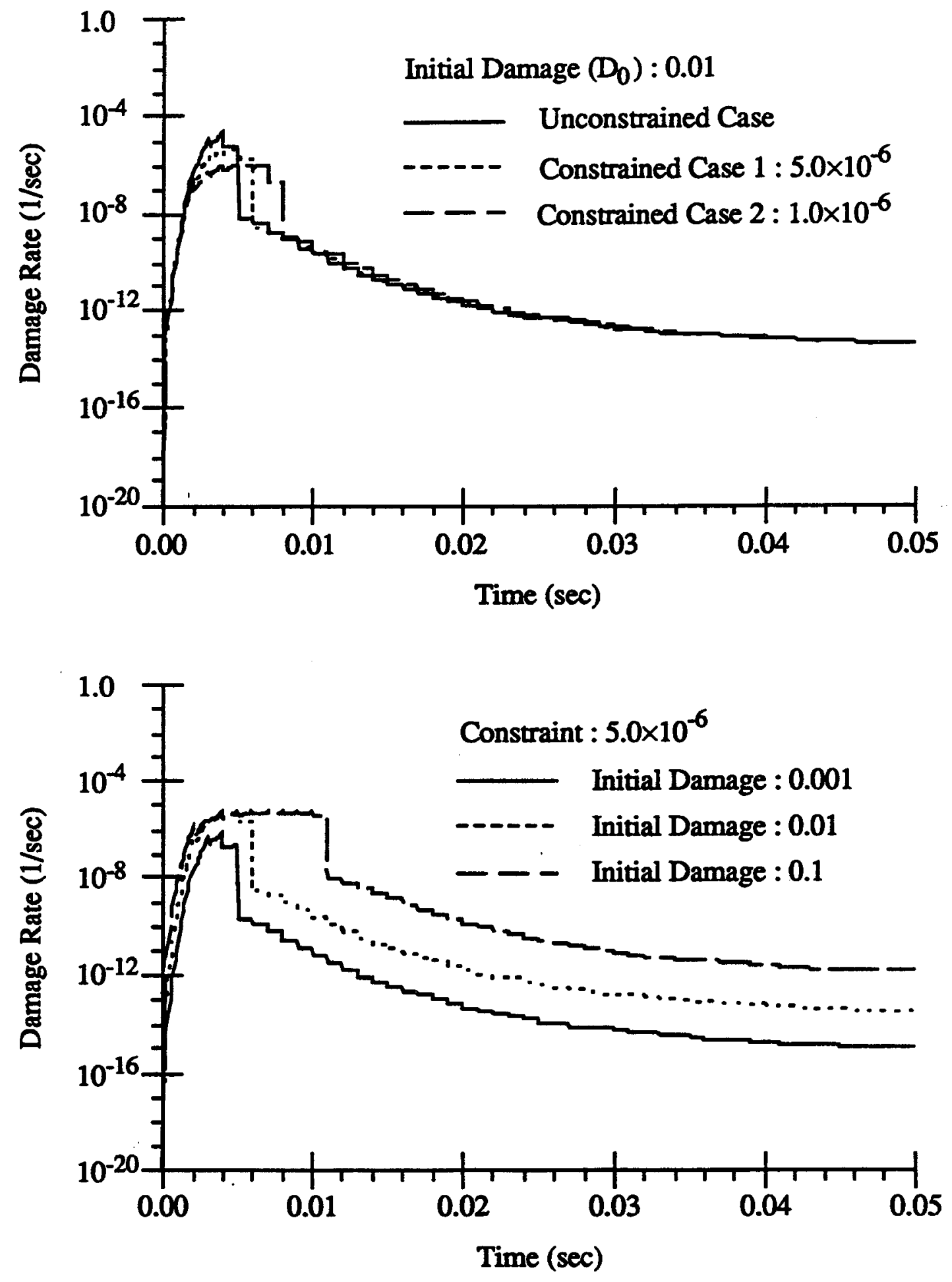

Figure 5.13 Transient Responses of Damage Rate 

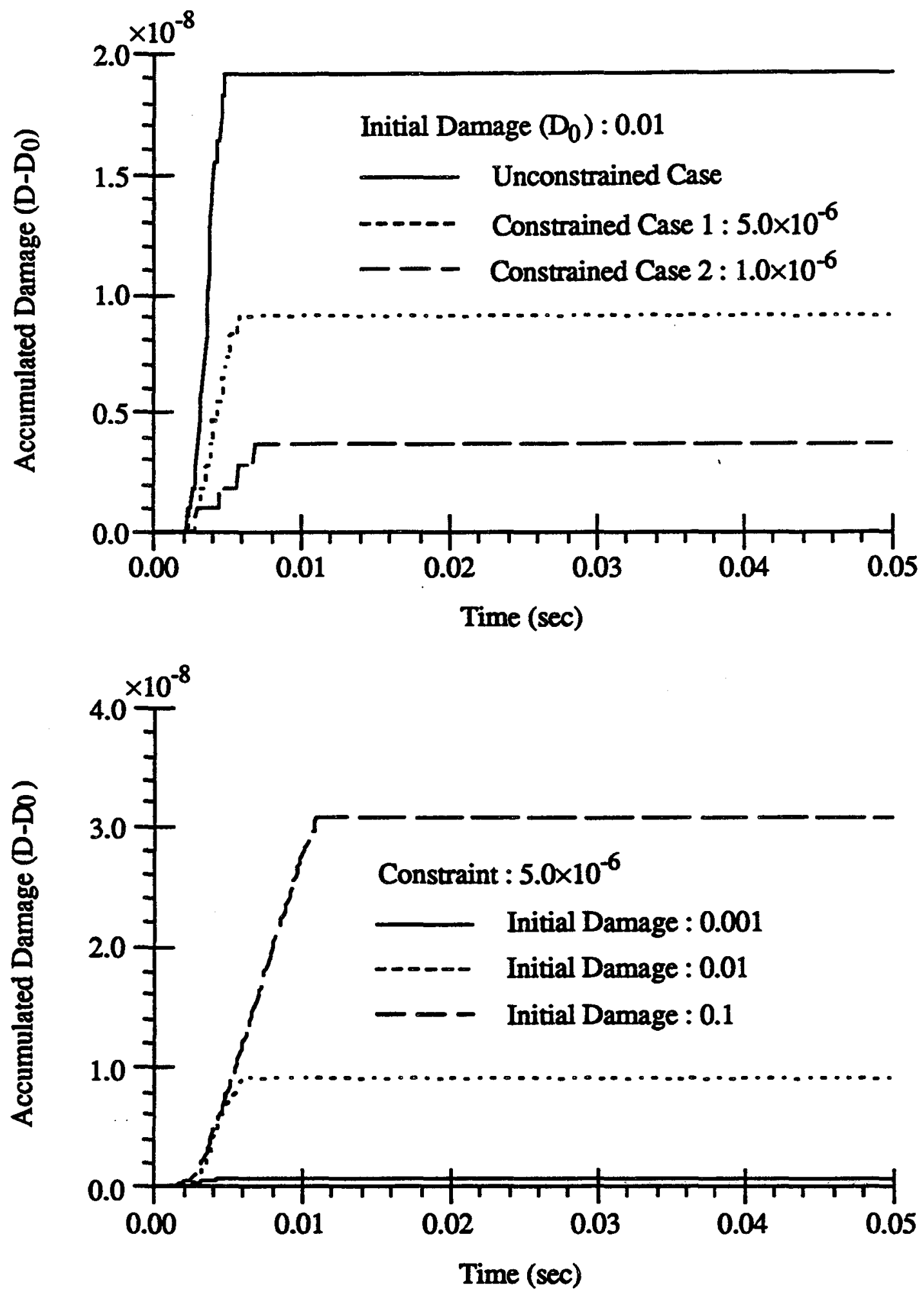

Figure 5.14 Transient Responses of Accumulated Damage 


\section{CHAPTER 6 \\ SUMMARY, CONCLUSIONS AND RECOMMENDATIONS FOR FUTURE RESEARCH}

The theme of the research in damage-mitigating control of mechanical systems is summarized as follows:

- For control of mechanical systems, damage prediction and damage mitigation are carried out based on the available sensory and operational information such that the plant can be inexpensively maintained, and safely and efficiently steered under diverse operating conditions.

- High performance is retained without overstraining the mechanical structures such that the functional life of critical components is increased resulting in enhanced safety, operational reliability, and availability.

In this report, a fatigue damage model has been developed in the continuoustime setting such that this damage model can be integrated with the plant dynamic model for the purpose of control synthesis. A procedure of synthesizing an optimal policy for open-loop control has been formulated via nonlinear programming under the constraints of damage rate and accumulated damage. Finally, efficacy of the proposed damage-mitigating control system has been demonstrated via simulation of the transient operations of a reusable rocket engine.

This research in damage-mitigating control is interdisciplinary and addresses the fields of active control technology and structural integrity. Extended service life coupled with enhanced safety and high performance will have a significant economic impact in diverse industrial applications. Examples include reusable rocket engines for space propulsion, rotating and fixed wing aircraft, fossil and nuclear plants for electric power generation, automotive and truck engine/transmission systems, and large rolling mills. Furthermore, as the science and technology of materials evolve, the updated damage characteristics of the structural components can be systematically incorporated within the framework of the proposed control system.

\subsection{Recommendations for Future Research}

The structure of the proposed damage-mitigating control system is built upon the concepts of both the conventional state feedback control systems and fatigue life prediction. Certain assumptions and approximations are made in the modeling of damage dynamics for practicality of implementation. Further research is necessary for more accurate damage prediction of the critical components. In the synthesis of robust damage-mitigating control, research must be conducted for accommodation of the disturbances and errors in modeling of both plant dynamics and damage. The following topics of research are recommended in the areas of damage prediction and control. 


\section{Identification of material parameters and verification of the damage model}

The continuous-time damage model is built upon the strain-life approach which assumes that the coefficients for material properties are constant and cyclic stress-strain behavior remains stabilized for most of the fatigue life. The computation load for realtime damage control could be reduced by assuming the parameters of the structural materials to be invariant relative to temperature. However, if a service component is exposed to high temperature, then the effects of parametric variations and additional phenomena of damage such as creep and corrosion must be taken into consideration.

Apparently no consistent definition of damage exists in the current literature. Therefore, damage must be precisely defined for accurate prediction of service life especially if the synergistic effects of fatigue and other phenomena such as creep and corrosion are considered. In this research, the linear damage for a given stress or strain amplitude has been defined on the basis of the total number of cycles to failure. This definition has been extended for nonlinear damage where the concepts of normalized crack length and crack propagation have been used to identify the $\gamma$-parameter of the damage curve. Although this model of damage prediction is considered to be adequate for a certain range of service life, it may not be sufficiently accurate for the entire range because of the different mechanisms of crack initiation and growth. For an appropriate damage definition, experimental research is needed for a better understanding of the micro-structure and failure mechanisms of the structural materials.

\section{Creep-fatigue interaction and formulation of a multi-dimensional damage vector}

The damage equations developed in the main text of this report consider only the fatigue failure of the materials. If the service components are exposed to high temperature or gaseous environment, the creep or corrosion may play an important role in the failure of the materials. The damage prediction has to incorporate the effect of creep-fatigue interaction for more accurate results. A general structure for modeling of creep-fatigue and corrosion-fatigue failure is proposed in Appendix E. If the creep or corrosion damage becomes important, or several critical points for failure are considered, then the damage vector should be multi-dimensional and needs to be optimized to achieve the best trade-off between the damage of individual critical components and the system performance.

\section{Stochastic modeling of damage dynamics}

In contrast to the linear damage rule, the nonlinear damage model developed in Chapter 4 requires the information of initial damage to initiate the damage computation. The initial damage in a specific component, however, is difficult to be identified because the size, distribution, and geometry of the defects in the material may vary over a wide range. Since a large part of the fatigue life may be consumed during the initial stage of so called crack initiation especially for the components with smooth geometry or under light load, a small error in the estimation of initial damage may cause a significant difference in the fatigue life prediction. Other parametric and nonparametric uncertainties in the damage model such as those resulting from variations in the material properties also contribute to the inaccuracy and scattering in prediction of the service life. If an average value is used in the deterministic fatigue damage model, the variance of the predicted life is likely to be large and therefore the damage model 
would have poor repeatability. However, the modeling uncertainties could be directly taken into account if the damage dynamics are modeled by stochastic differential equations. This approach could minimize the modeling error in a statistical sense and be used in fatigue failure prognosis and risk analysis.

\section{Closed-loop control}

The open-loop control policy optimizes the system performance under the damage constraints without considering the plant modeling errors, disturbances, and sensor noise. The closed-loop control is required to maintain the system along the desired trajectory or at the normal steady-state operating condition. A closed loop system could be designed on the basis of the linearized plant model at the normal operating point by using the techniques of robust control synthesis such as LQG/LTR, $\mathrm{H}_{2} / \mathrm{H}_{\infty}$, or $\mu$-synthesis. However, to ensure that the feedback control does not violate the damage constraints, a two-tier structure of the control system might be needed. The concept of this hierarchically structured closed-loop control system is outlined in Appendix A. 


\section{APPENDIX A}

\section{CLOSED LOOP CONTROL}

Nonlinear programming generates an open-loop control policy to achieve optimal performance under the specified constraints of damage rate and accumulated damage as described in Chapter 3. However, because of plant modeling uncertainties (including unmodeled dynamics), sensor noise and disturbances, the actual plant response shall deviate from that of the modeled system when the plant is excited by the sequence of open-loop control commands. Therefore, a closed-loop control system is necessary to compensate for these deviations, and a state feedback controller may serve this purpose. If all plant states are not measurable or if the sensor data are noisecontaminated, a state estimator (e.g., a minimum variance filter) is necessary to obtain an estimate of the plant state vector. If the deviations from the nominal trajectory are not large, the state feedback and state estimator gain matrices could be synthesized based on a linearized model of the plant. However, if the plant is required to be operated over a wide range (for example, scheduled shutdown of a power plant from full power), then linearization must be carried out at several operating points and the closed loop control could be made piecewise linear by adopting the concept of gain scheduling. Should this concept prove to be inadequate, more advanced techniques of (model reference or self tuning) adaptive control [Goodwin and Sin (1984)] and reconfigurable control [Stengel (1991)] need to be considered.

A block diagram of the closed loop damage control and decision system is proposed in Figure A.1 where $\{\mathrm{uff}\},\{\mathbf{x f f}\},\left[\mathrm{vff}^{\mathrm{ff}}\right\}$ and $[\dot{\mathrm{v}} \mathrm{ff}\}$ represent the sequences of the plant input, plant state, accumulated damage, and damage rate, respectively, generated as an optimal solution of the open-loop control problem via nonlinear programming, and $[y \mathrm{ff}\}$ is the resulting sequence of plant output, which is obtained as a nonlinear function $\mathrm{g}(\bullet)$ of the plant state vector $\mathrm{xff}$. As mentioned above, a feedback controller is necessary to compensate for the plant modeling uncertainties and disturbances such that the trajectory of the actual plant output $y$ should be close to that of the desired plant output yff which serves as the reference trajectory. The resulting error in the plant output is an input to the state estimator, and the feedback control signal ufb compensates for errors resulting from plant disturbances and sensor noise. The estimated state $\hat{\mathbf{x}}$ which is obtained as the sum of the estimated state error and the reference state $\mathrm{xff}$ is fed to the structural model. The output of the structural model is the load vector $q$ which contains stress, strain and other information necessary for damage assessment. Some of the elements of the load vector $q$ (e.g., strain and temperature at the critical points) may be directly measurable as indicated in Figure A.1 by additional measurements $\check{y}$.

The closed-loop control system is partitioned into two modules. The state feedback control law in the first module could be formulated by using the established techniques of robust multi-input multi-output (MMMO) control synthesis, which rely on approximation of the plant dynamics by a linear time-invariant model (e.g., $\mathrm{H}_{2}$-based LQG/LTR [Stein and Athans (1987)], $\mathrm{H}_{2} / \mathrm{H}_{\infty}$ optimization [Doyle et al. (1989)] or $\mu$ synthesis [Doyle (1982); Doyle et al. (1982)]). However, if the plant dynamics cannot be approximated by piecewise linearization or time-averaging of the varying parameters, then selection of the control synthesis technique will depend upon the 
specific application. The second module is a nonlinear controller which contributes to damage reduction in the critical plant components under feedback control and also serves to generate early warnings and prognoses of impending failures of the critical plant components. Since the damage rate $\dot{v}$ in the closed-loop control system may violate the specified constraints due to the additional compensation generated by the feedback control effort $\mathrm{u}^{\mathrm{fb}}$, a nonlinear controller is incorporated into the system to reduce the damage rate $\dot{v}$. This is accomplished by corrections $x^{c o r}$ in the reference trajectory and $\mathrm{ucor}$ in the control effort. The rationale for modifying the reference trajectory $\{x \mathrm{ff}\}$ is that, due to plant disturbances and sensor noise, it may not be possible to follow this trajectory without violating the damage constraints. The additional control effort ucor is intended to provide a fast corrective action to the control input $u_{k}$ whenever necessary. The nonlinear control system in the outer loop, as shown in Figure A.1, serves two purposes, namely, (i) trimming of the linear feedback control signal to maintain the damage rate or accumulated damage rate vector within the limits, and (ii) modification of the tracking signal (i.e., the reference signal) to circumvent the problem of exceeding the damage rate limits, which may result from plant modeling errors, uncertainties and disturbances. A possible approach to synthesis of the nonlinear damage controller is first to postulate a mathematical structure of the controller and then optimize the controller parameters relative to a cost functional that would penalize:

- Plant state and damage rate over the task period;

- Final plant state and the accumulated damage.

The outputs of the damage controller, namely $x^{\text {cor }}$ and $u^{\text {cor }}$ in Figure A.1, need to be constrained to be norm-bounded to assure the system stability; $x$ cor and $u$ cor can also be considered as exogenous inputs to the linear robust control system in the inner loop. Therefore, the $\mathrm{H}_{\infty}$ bounds on $\mathrm{xcor}$ and $\mathrm{ucor}$ can be fine-tuned to satisfy the specified requirements of performance and stability robustness in the $\mu$-synthesis procedure.

Since the damage model is highly nonlinear and is very sensitive to modeling uncertainties [Ray et al. (1993a); (1993b)], a combination of adaptive control and linear robust control techniques [Kidd (1991)] is also a viable option. However, such techniques are often restricted to single-input single-output (SISO) systems. The damage mitigation problem is MIMO because of the multiple phenomena (e.g., fatigue, corrosion, and creep) that may simultaneously occur at several critical points of the structure. Therefore, both systems-theoretic and heuristic techniques should be explored for synthesizing the nonlinear controller. 


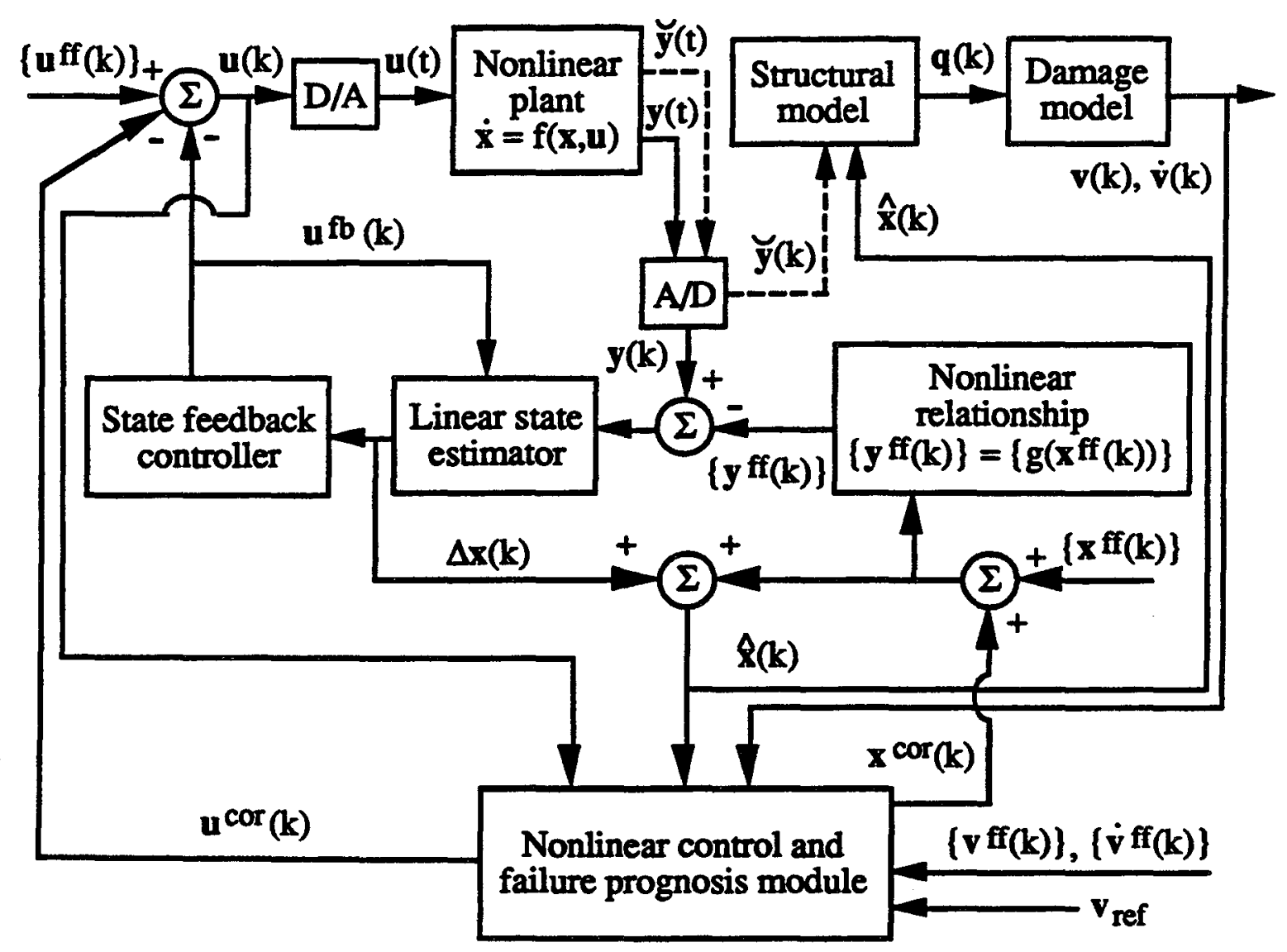

Figure A.1 Closed-Loop Damage Control System 


\section{APPENDIX B}

\section{RAINFLOW CYCLE COUNTING METHOD}

The load history in a real application is usually of varying amplitude and frequency. The fatigue damage analysis due to spectral loading is more complicated than that due to the constant amplitude of load. The purpose of cycle counting is to identify the cycles out of the spectral load history so that the strain-life data obtained from the experiments can be applied to the fatigue life prediction. The cycle counting methods, such as range-pair, rainflow, and racetrack are available in the literature [Fuchs and Stephens (1980)]. Among those methods, rainflow has been shown to be superior and yields the best fatigue-life prediction [Dowling (1983)]. The rainflow counting method which has been adopted in this report is briefly described below.

Figure B.1 shows the application of rainflow counting on a spectral load history. The stress-time curve is plotted with stress as the abscissa and time as the ordinate. Imagine that a raindrop is released at each peak point, flows along the direction of stress reversal, and then drops down. Starting from the first peak point and injecting the raindrops in a timed sequence, the following rules can be applied:

(1) If the raindrop is released from the left peak point and flows to the right, the flow stops when it comes to a point where there is another peak point in the same horizontal level which is farther left than, or at the same stress level with the starting point.

(2) If the raindrop is released from the right peak point and flows to the left, the flow stops when it comes to a point where there is another peak point in the same horizontal level which is farther right than, or at the same stress level with the starting point.

(3) The flow stops when it is blocked by a previous flow path.

In Figure B.1, the first rule is applied for the flows starting at point 2, 4 and 6. For example, the flow 2-3-4' stops at the point 4 ' since 4 ' is on the left of the starting point 2. Similarly, the second rule is applied for the flows starting from the points 1 and 5 . The flows from 3 and 7 stop at $2^{\prime}$ and $6^{\prime}$ due to the third rule. As can be seen from Figure B.1, a small cycle, 6-7-6', is extracted from a bigger cycle, 5-8-5'. After a cycle is closed, it can be discarded from the load history. Similarly, the cycles, 5-8-5' and 23-2', are extracted from the biggest cycle, 1-4-1'. Therefore, the large cycles in the load history are always retained and those cycles are most significant for fatigue damage prediction. The small cycles could be treated as the intermediate interruption. 


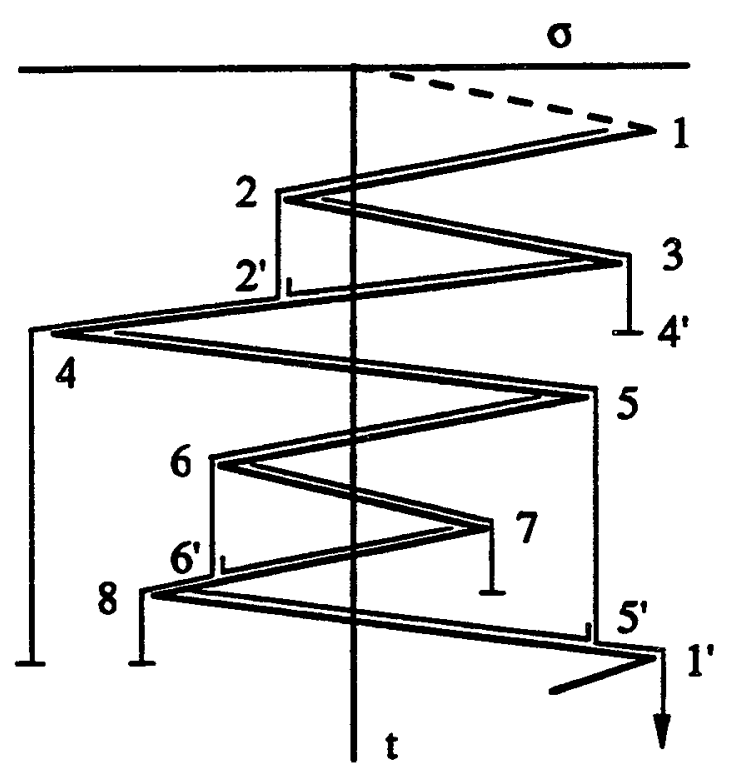

Figure B.1 Rainflow Cycle Counting Method 


\section{APPENDIX C}

\section{MODELING OF PLANT DYNAMICS}

The plant under consideration in this report has been represented by a simplified model of a bipropellant rocket engine as shown in Figure 5.1. The physical process of the rocket engine consists of distributed dynamic elements which are approximately represented by nonlinear ordinary differential equations with time as the independent variable. A model solution diagram [Ray et al. (1980)] for the bipropellant rocket engine is shown in Figure C.1. Each block represents a physical plant component or a group of components. The lines interconnecting the blocks indicate the direction of information flow. The inputs to the plant are the commands to two oxygen valves, which control the liquid oxygen flowing into the preburner and the main combustor.

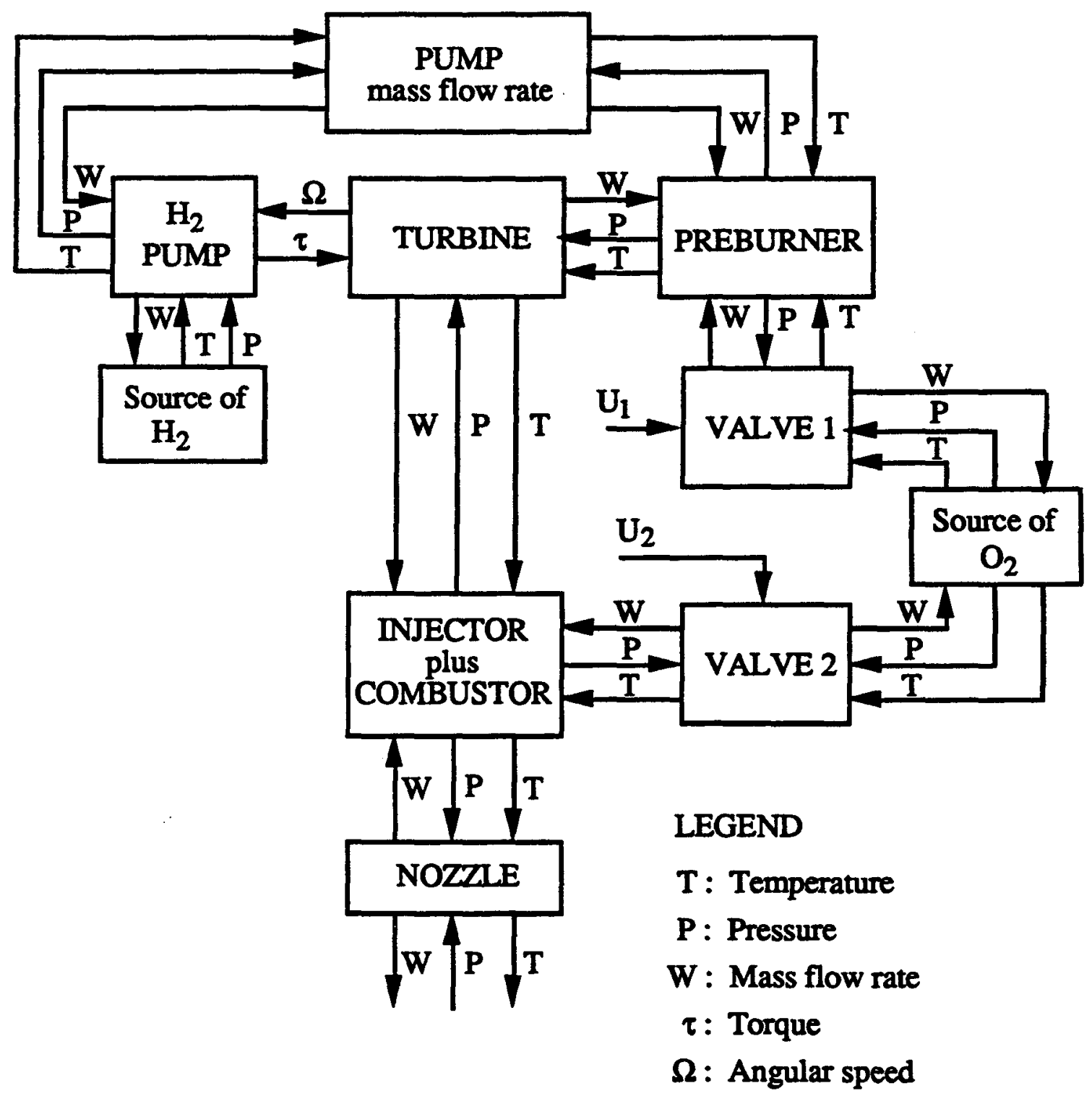

Figure C.1 Model Solution Diagram of a Bipropellant Rocket Engine 
The two controlled outputs in Figure C.1 are the main combustor pressure and the ratio of the oxygen flow and hydrogen flow. Other outputs such as turbine shaft speed and torque are also required to predict the damage occurring at the turbine blade. This model consists of eight state variables, namely turbine shaft speed, pump $\left(\mathrm{LH}_{2}-\right.$ fuel) mass flow rate, preburner gas pressure, preburner gas density, combustor gas pressure, combustor gas density, and the two flow rates of oxidant.

The model equations are formulated by using approximation of the integral forms of the conservation relations for mass, momentum, and energy, semi-empirical relationships for fluid flow and heat transfer, and state relations for thermodynamic properties of the working fluids. Model parameters are evaluated from the design and experimental data of the manufacturer [NASA (1991)]. Major assumptions in addition to the lumped parameter approximation of the model equations are:

(1) Uniform fluid flow over the pipe cross-section;

(2) No heat gain or loss for the fluid flowing through the pipes;

(3) Perfect thermal insulation between plant components and the environment;

(4) Negligible pressure drop due to velocity and gravitational heads in the fluid paths;

(5) Constant density of the oxygen and hydrogen in the liquid phase;

(6) Validity of perfect gas law inside the preburner and main combustor;

(7) Choked flow through the turbine and nozzle; and

(8) Representation of the valve dynamics by a first order lag.

The following subsections formulate the governing equations of the plant components including the liquid hydrogen pump, pump turbine, liquid hydrogen pipeline, two oxygen inlet valves, preburner and main combustor as shown in Figure c.1.

The control input, measured output, and plant state variables are listed in Table C.1 along with their steady-state values at two operating conditions. The system matrices and the eigenvalues of the linearized state space model at two steady-state operating conditions are listed in Table C.2.

\section{Pump Model:}

The pump driven by the turbine feeds pressurized liquid hydrogen into the preburner. The empirical relation describing the pump head-flow-speed characteristics [Hicks and Edwards (1971); Ray (1976)] is given as

$$
\frac{\Delta P_{p m p}}{\rho_{h} \Omega_{p m p}{ }^{2}}=f\left(\frac{W_{p m p}}{\rho_{h} \Omega_{p m p}}\right)
$$

where $\Delta P_{p m p}$ is the pressure increase between the inlet and outlet of the pump, $W_{p m p}$ and $P_{h}$ are the mass flow rate and mass density of liquid hydrogen, respectively, and 
$\Omega_{\mathrm{pmp}}$ is the pump speed. Eq. (C.1) can be normalized with respect to a normal operating point (which is characterized by the values of the process variables marked by the superscript ${ }^{*}$ ) such that

$$
\frac{\Delta P_{p m p} /\left(P_{h} \Omega_{p m p}{ }^{2}\right)}{\Delta P^{*} /\left(p^{*} \Omega^{* 2}\right)}=f\left(\frac{W_{p m p} / P_{h} \Omega_{p m p}}{W^{*} / P^{*} \Omega^{*}}\right)
$$

Assuming that the density of liquid hydrogen remains constant within the operating range, the pump characteristics described in eq. (C.2) can be approximated by a second order polynomial based on the manufacturer's data [NASA (1991)] as:

$$
\frac{\Delta P_{p m p} / \Delta P^{*}}{\left(\Omega_{p m p} / \Omega^{*}\right)^{2}}=a_{2}\left(\frac{W_{p m p} / W^{*}}{\Omega_{p m p} / \Omega^{*}}\right)^{2}+a_{1}\left(\frac{W_{p m p} / W^{*}}{\Omega_{p m p} / \Omega^{*}}\right)+a_{0}=y
$$

The mass flow rate, $W_{p m p}$, of liquid hydrogen and the angular speed, $\Omega_{\mathrm{pmp}}$, of the turbo-pump are state variables. The other coefficients, $\mathrm{a}_{0}, \mathrm{a}_{1}, \mathrm{a}_{2}$ and the variables at normal operating point are given as pump data. Then, the pressure head, $\Delta \mathrm{P}_{\mathrm{pmp}}$, and the pump outlet pressure, $P_{d e l}$, are computed by

$$
\begin{aligned}
\Delta \mathrm{P}_{\mathrm{pmp}} & =\Delta \mathrm{P}^{*}\left(\Omega_{\mathrm{pmp}} / \Omega^{*}\right)^{2} \mathrm{y} \\
\mathrm{P}_{\text {del }} & =\Delta \mathrm{P}_{\mathrm{pmp}}+\mathrm{P}_{\text {suc }}
\end{aligned}
$$

where the pump inlet pressure, $P_{\text {suc, }}$, is assumed to be constant and equal to the pressure at the source of liquid hydrogen. Once $\Delta \mathrm{P}_{\mathrm{pmp}}$ is known, the output power of the pump can be obtained as:

$$
\text { Output power }=\Delta P_{p m p} \frac{W_{p m p}}{P_{h}}
$$

The input power and the torque $\tau_{\mathrm{pmp}}$ required to drive the pump are then computed from the pump efficiency $\eta_{\text {pmp }}$ as:

$$
\text { Input power }=\Delta P_{p m p} \frac{W_{p m p}}{P_{h} \eta_{p m p}} \quad \text { and } \quad \tau_{p m p}=\Delta P_{p m p} \frac{W_{p m p}}{P_{h} \eta_{p m p} \Omega_{p m p}}
$$

where the efficiency is also approximated by a second order polynomial based on the manufacturer's data [NASA (1991)] as:

$$
\eta_{\mathrm{pmp}}=\alpha_{2}\left(\frac{\mathrm{W}_{\mathrm{pmp}} / \mathrm{W}^{*}}{\Omega_{\mathrm{pmp}} / \Omega^{*}}\right)^{2}+\alpha_{1}\left(\frac{\mathrm{W}_{\mathrm{pmp}} / \mathrm{W}^{*}}{\Omega_{\mathrm{pmp}} / \Omega^{*}}\right)+\alpha_{0}
$$

Assuming perfect insulation between the pump and the environment, the energy loss is completely transformed into heat and causes the increase of enthalpy (and temperature) for the liquid hydrogen as presented below: 


$$
\begin{aligned}
& \Delta \mathrm{H}_{\mathrm{pmp}}=\frac{\text { Input power }- \text { Output power }}{\mathrm{W}_{\mathrm{pmp}}}=\frac{\Delta \mathrm{P}_{\mathrm{pmp}}}{\mathrm{J} \mathrm{P}_{\mathrm{h}}}\left(\frac{1}{\eta_{\mathrm{pmp}}}-1\right) \text { and } \\
& \Delta \mathrm{T}_{\mathrm{pmp}}=\frac{\Delta \mathrm{P}_{\mathrm{pmp}}}{\mathrm{J} \mathrm{P}_{\mathrm{h}} \mathrm{C}_{\mathrm{ph}}}\left(\frac{1}{\eta_{\mathrm{pmp}}}-1\right)
\end{aligned}
$$

where $J$ is the conversion factor from the thermal unit to the work unit, and $C_{p h}$ is the specific heat of liquid hydrogen at constant pressure.

Pipeline Model for Liquid Hydrogen Flow:

For the liquid hydrogen flowing over the pipeline, the head loss occurs due to friction along the wall of the pipeline. Let $A$ and $\ell$ be the average area of cross section and the length of the pipeline respectively. Then, the pipeline model is formulated by approximating the integral form of the momentum equation as:

$$
\begin{aligned}
& \frac{d}{d t}\left(P_{h} \ell A v\right)=P_{d e l} A-P_{p b r} A-F_{\text {fric }} \\
\Rightarrow & \frac{\ell}{A} \frac{d}{d t}\left(P_{h} A v\right)=P_{d e l}-P_{p b r}-\Delta P_{\text {fric }} \\
\Rightarrow & \frac{\ell}{A} \frac{d W_{p m p}}{d t}=P_{d e l}-P_{p b r}-\Delta P_{\text {fric }}
\end{aligned}
$$

where $\mathrm{P}_{\mathrm{pbr}}$ is the pressure of the preburner and the frictional pressure drop is obtained as [Shames (1962)]:

$$
\Delta P_{\text {fric }}=K_{\text {fric }} \frac{I W_{p m p} I W_{\text {pmp }}}{\rho_{h}}
$$

The loss of heat from the pipe line is assumed to be balanced by the gain of heat due to friction. Thus, the preburner inlet temperature is taken to be equal to the pump outlet temperature.

\section{Oxygen Inlet Valve Models:}

Two oxygen valves control the oxidant flow into the preburner and combustor. Let $P_{0}$ be the pressure inside the oxygen tank. The mass flow rates of oxygen are computed from the orifice equation [Blackburn et al. (1960)] as

$$
\begin{aligned}
\mathrm{W}_{\mathrm{pbr}} & =\mathrm{K}_{\mathrm{pbr}} \xi_{\mathrm{pbr}} \sqrt{\left(\mathrm{P}_{\mathrm{o}}-\mathrm{P}_{\mathrm{pbr}}\right) \mathrm{P}_{\mathrm{o}}} \\
\mathbf{W}_{\mathrm{cmb}} & =\mathrm{K}_{\mathrm{cmb}} \xi_{\mathrm{cmb}} \sqrt{\left(\mathrm{P}_{\mathrm{o}}-\mathrm{P}_{\mathrm{cmb}}\right) \mathrm{p}_{\mathrm{o}}}
\end{aligned}
$$

where $p_{0}$ is the density of liquid oxygen, and $\xi_{\mathrm{pbr}}$ and $\xi_{\mathrm{cmb}}$ are the actual valve positions for the preburner and combustor respectively. The dynamics of the valves subject to the command inputs are assumed to behave as the first order lag such that 


$$
\begin{aligned}
\frac{\mathrm{d} \xi_{\mathrm{pbr}}}{\mathrm{dt}} & =\frac{\xi_{\mathrm{pbr}}-\mathrm{u}_{\mathrm{pbr}}}{\theta_{\mathrm{pbr}}} \\
\frac{\mathrm{d} \xi_{\mathrm{cmb}}}{\mathrm{dt}} & =\frac{\xi_{\mathrm{cmb}}-\mathrm{u}_{\mathrm{cmb}}}{\theta_{\mathrm{cmb}}}
\end{aligned}
$$

where $u_{\mathrm{pbr}}$ and $\mathrm{u}_{\mathrm{cmb}}$ are the electrical commands to the oxygen valves of preburner and combustor, and $\theta_{\mathrm{pbr}}$ and $\theta_{\mathrm{cmb}}$ are the time constants of the respective values.

\section{Turbine Model:}

For simplicity of modeling, choked flow [Blackburn et al. (1960)] is assumed through the turbine, and no loss of pressure and enthalpy occurs between the preburner and the turbine. Therefore, the turbine inlet pressure and temperature are assumed to be identical to the pressure and temperature in the preburner. Then, the mass flow rate of hot gas flowing through the turbine can be expressed by

$$
\mathbf{W}_{\mathrm{trb}}=\mathrm{K}_{\mathrm{trbc}} \frac{\mathrm{P}_{\mathrm{pbr}}}{\sqrt{\mathrm{T}_{\mathrm{pbr}}}}
$$

where $T_{p b r}$ is the absolute gas temperature in the preburner. For an isentropic process, the turbine exhaust pressure, $P_{t r b}$, and ideal outlet temperature, $T_{\text {trbi }}$, are related to the turbine inlet pressure, $\mathrm{P}_{\mathrm{pbr}}$, and inlet temperature, $\mathrm{T}_{\mathrm{pbr}}$, by

$$
P_{p b r}^{1-\gamma} T_{p b r}{ }^{\gamma}=P_{t b b}^{1-\gamma} T_{t r b l^{\gamma}}
$$

where $\gamma=C_{p} / C_{v}$ for the gas. Following the information flow in the model solution diagram in Figure $C .1$, the turbine exhaust pressure, $P_{t r b}$, is approximated by the combustor pressure plus the head loss due to friction through the injector tubes. A relationship similar to eq. (C.12) or (C.13) is used to obtain the mass flow and pressure drop in the injector (which connects the turbine exhaust with the combustor chamber):

$$
W_{t r b}=K_{t r b f} \sqrt{\left(P_{t r b}-P_{c m b}\right) P_{c m b}}
$$

The above eq. (C.18) has been approximated by using the density $\rho_{\mathrm{cmb}}$ of the combustor gas instead of the average gas density in the injector. The rationale for this approximation is that eqs. (C.16), (C.17) and (C.18) can be combined to obtain the turbine exhaust pressure $P_{\text {trbe }}$ via a closed form relation, and the ideal outlet temperature can be directly evaluated from eq. (C.17). The actual outlet temperature is estimated from the turbine efficiency, which is given by the following empirical equation [NASA (1991); Ray (1976)]:

$$
\eta_{\mathrm{trb}}=\eta^{*}\left(1-\alpha_{\mathrm{trb}}\left(\left[\frac{\Omega}{\Omega^{*}} / \sqrt{\frac{\Delta \mathrm{H}_{\mathrm{tbi}}}{\Delta \mathrm{H}^{*}}}\right]-1\right)^{2}\right)
$$


where $\eta^{*}, \Omega^{*}$ and $\Delta \mathrm{H}^{*}$ are the turbine efficiency, speed and ideal enthalpy drop at the designed operating condition. The turbine speed, $\Omega_{\text {trb }}$, is identical to the pump speed, and therefore is a state variable. The ideal enthalpy drop, $\Delta \mathrm{H}_{\text {urbi }}$, is given by

$$
\Delta \mathrm{H}_{\mathrm{trbi}}=\mathrm{C}_{\mathrm{ppbr}}\left(\mathrm{T}_{\mathrm{pbr}}-\mathrm{T}_{\mathrm{trbi}}\right)
$$

The actual enthalpy drop is then obtained as $\Delta H_{t r b}=\Delta H_{t r b i} \eta_{\text {trb }}$. Since the difference between the ideal enthalpy drop and the actual enthalpy drop is converted to irreversible loss of energy and no other loss is assumed to be prevalent, the turbine torque $\tau_{\text {trb }}$ is obtained as

$$
\tau_{\text {trb }}=W_{\text {trb }} \Delta H_{\text {trb }} / \Omega_{\text {pmp }}
$$

where $\Omega_{\mathrm{pmp}}$ is the angular speed of the turbo-pump. Having known $\tau_{\text {trb }}$, the equation of motion for the turbine is given from conservation of angular momentum [Shames (1962)]:

$$
I_{p m p} \frac{d \Omega_{p m p}}{d t}=\tau_{t r b}-\tau_{p m p}
$$

where $I_{p m p}$ is the moment of inertia of the turbo-pump assembly.

\section{Combustor Model:}

The combustor model is formulated via approximation of the integral form of conservation of mass flow and internal energy. Assuming the gas inside the main combustor chamber to be perfect, the variables describing the thermodynamic states in the combustor chamber are related by the ideal gas law [Zemansky and Van Ness (1966)]:

$$
P_{c m b}=R \rho_{c m b} T_{c m b}
$$

where $R$ is the characteristic gas constant. In the model, the pressure, $P_{c m b}$, and the density, $\rho_{\mathrm{cmb}}$, are selected as the state variables. The absolute temperature, $T_{\mathrm{cmb}}$, of the combustor gas is computed from eq. (C.23) as a function of these two state variables. The exhaust hot gas, which generates the thrust for the rocket engine, is obtained as the choked flow through the nozzle. Then, the mass flow leaving the combustor can be computed as

$$
\mathrm{W}_{\mathrm{noz}}=\mathrm{K}_{\mathrm{noz}} \frac{\mathrm{P}_{\text {com }}}{\sqrt{\mathrm{T}_{\mathrm{cmb}}}}
$$

Having known the nozzle exhaust flow from eq. (C.24), the gas density inside the combustor chamber, $\rho_{\mathrm{cmb}}$ is computed from the conservation of mass such that

$$
\frac{d P_{c m b}}{d t}=\frac{W_{t b b}+W_{c m b}-W_{\text {noz }}}{V_{c m b}}
$$


where $V_{c m b}$ is the volume of the main combustor chamber. Assuming prefect insulation without any energy loss to the environment, the conservation of internal energy yields:

$$
\frac{d}{d t}\left(C_{v} V P T\right)_{c m b}=W_{t r b} H_{t r b}+W_{c m b} C_{p o} T_{o}-W_{n o z} C_{p c m b} T_{c m b}+C_{f u e l} W_{c m b}
$$

where the last term, $\mathrm{C}_{\text {fuel }} \mathrm{W}_{\mathrm{cmb}}$, is the heat generated from consumption of the oxidant in the fuel-rich environment. Substituting $\rho T$ by $P / R$ from the ideal gas law, eq. (C.26) can be written as

$$
\frac{d P_{c m b}}{d t}=\frac{\left(W_{t r b} H_{t r b e}+W_{c m b} C_{p o} T_{0}-W_{n o z} C_{p c m b} T_{c m b}+C_{f u e l} W_{c m b}\right)}{C_{v c m b} V_{c m b}} R
$$

\section{Preburner Model:}

The preburner model is formulated similar to the combustor model with the gas density, $\rho_{\mathrm{pbr}}$, and the pressure, $\mathrm{P}_{\mathrm{pbr}}$, as the state variables. The absolute gas temperature is computed from the ideal gas law. From the conservation of mass and energy, two equations similar to eqs. (C.25) and (C.27) are given as

$$
\begin{aligned}
& \frac{d P_{p b r}}{d t}=\frac{W_{p m p}+W_{p b r}-W_{t r b}}{V_{p b r}} \\
& \frac{d P_{p b r}}{d t}=\frac{W_{p m p} H_{p m p}+W_{p b r} C_{p o} T_{o}-W_{t r b} H_{t r b i}+C_{f u e l} W_{p b r}}{C_{v p b r} V_{p b r}} R
\end{aligned}
$$


Table C.1 Plant Control Input, Measured Output and State Variables

Steady-state values for combustor pressure $2700 \mathrm{psi} \quad 3000 \mathrm{psi}$

Input variables:

$\mathrm{u}_{1}$ : Command to the preburner $\mathrm{O}_{2}$ valve 0.655466 0.838256 (dimensionless)

$\mathrm{u}_{2}$ : Command to the combustor $\mathrm{O}_{2}$ valve (dimensionless)

$\begin{array}{ll}0.697910 & 0.807391\end{array}$

Measured output variables:

$\mathbf{y}_{1}$ : Combustor gas pressure (psi)

2700

3000

$\mathrm{y}_{2}: \mathrm{O}_{2} / \mathrm{H}_{2}$ ratio

6.02

6.02

State variables:

$\mathrm{x}_{1}$ : Turbine (pump) shaft speed (rad/sec) 3606.40 3904.71

$\mathrm{x}_{2}: \mathrm{LH}_{2}$-fuel mass flow rate (lbm/sec)

115.363

128.206

$\mathrm{x}_{3}$ : Preburner gas pressure (psi)

4240.40

$\mathrm{X}_{4}$ : Preburner gas density $\left(\mathrm{lbm} / \mathrm{in}^{3}\right)$

X5 : Combustor gas pressure (psi)

$0.3207 \times 10^{-3}$

$0.3571 \times 10^{-3}$

$\mathrm{x}_{6}$ : Combustor gas density (lbm/in 3 )

2700.00 3000.00

$\mathrm{x}_{7}$ : Position of the preburner $\mathrm{O}_{2}$ valve (dimensionless)

$0.1038 \times 10^{-3} \quad 0.1153 \times 10^{-3}$

0.655466

0.838256

$\mathrm{x}_{8}$ : Position of the combustor $\mathrm{O}_{2}$ valve (dimensionless)

0.697910

0.807391 
Table C.2 System Matrices for the Linearized Plant Model

$$
\text { System equations : } \quad \begin{aligned}
& \dot{\mathbf{x}}=\mathbf{A x}+\mathbf{B u} \\
& \mathbf{y}=\mathbf{C x}+\mathbf{D u}
\end{aligned}
$$

System matrices for combustor pressure at $3000 \mathrm{psi}$ :

$$
\begin{aligned}
& \mathrm{A}=\left[\begin{array}{cccccc}
-.1685 \mathrm{D}+02 & -.6154 \mathrm{D}+02 & 0.4105 \mathrm{D}+02 & -.1240 \mathrm{D}+09 & -.4582 \mathrm{D}+02 & 0.2544 \mathrm{D}+09 \\
0.6064 \mathrm{D}+02 & -.6002 \mathrm{D}+03 & -.2000 \mathrm{D}+02 & 0.0000 \mathrm{D}+00 & 0.0000 \mathrm{D}+00 & 0.0000 \mathrm{D}+00 \\
0.1215 \mathrm{D}+02 & 0.9678 \mathrm{D}+03 & -.1655 \mathrm{D}+04 & 0.3953 \mathrm{D}+10 & 0.0000 \mathrm{D}+00 & 0.0000 \mathrm{D}+00 \\
0.0000 \mathrm{D}+00 & 0.8333 \mathrm{D}-03 & -.3547 \mathrm{D}-04 & -.2382 \mathrm{D}+03 & 0.0000 \mathrm{D}+00 & 0.0000 \mathrm{D}+00 \\
0.2268 \mathrm{D}+01 & 0.0000 \mathrm{D}+00 & 0.2210 \mathrm{D}+03 & -.9147 \mathrm{D}+09 & -.4586 \mathrm{D}+04 & 0.3122 \mathrm{D}+11 \\
0.0000 \mathrm{D}+00 & 0.0000 \mathrm{D}+00 & 0.5349 \mathrm{D}-05 & 0.6351 \mathrm{D}+02 & -.5675 \mathrm{D}-04 & -.8664 \mathrm{D}+03 \\
0.0000 \mathrm{D}+00 & 0.0000 \mathrm{D}+00 & 0.0000 \mathrm{D}+00 & 0.0000 \mathrm{D}+00 & 0.0000 \mathrm{D}+00 & 0.0000 \mathrm{D}+00 \\
0.0000 \mathrm{D}+00 & 0.0000 \mathrm{D}+00 & 0.0000 \mathrm{D}+00 & 0.0000 \mathrm{D}+00 & 0.0000 \mathrm{D}+00 & 0.0000 \mathrm{D}+00
\end{array}\right. \\
& \left.\begin{array}{ll}
0.0000 \mathrm{D}+00 & 0.0000 \mathrm{D}+00 \\
0.0000 \mathrm{D}+00 & 0.0000 \mathrm{D}+00 \\
0.3206 \mathrm{D}+07 & 0.0000 \mathrm{D}+00 \\
0.7564 \mathrm{D}-01 & 0.0000 \mathrm{D}+00 \\
0.0000 \mathrm{D}+00 & 0.8116 \mathrm{D}+07 \\
0.0000 \mathrm{D}+00 & 0.1915 \mathrm{D}+00 \\
-.3333 \mathrm{D}+03 & 0.0000 \mathrm{D}+00 \\
0.0000 \mathrm{D}+00 & -.3333 \mathrm{D}+03
\end{array}\right] \\
& B=\left[\begin{array}{ll}
0.0000 \mathrm{D}+00 & 0.0000 \mathrm{D}+00 \\
0.0000 \mathrm{D}+00 & 0.0000 \mathrm{D}+00 \\
0.0000 \mathrm{D}+00 & 0.0000 \mathrm{D}+00 \\
0.0000 \mathrm{D}+00 & 0.0000 \mathrm{D}+00 \\
0.0000 \mathrm{D}+00 & 0.0000 \mathrm{D}+00 \\
0.0000 \mathrm{D}+00 & 0.0000 \mathrm{D}+00 \\
0.3333 \mathrm{D}+03 & 0.0000 \mathrm{D}+00 \\
0.0000 \mathrm{D}+00 & 0.3333 \mathrm{D}+03
\end{array}\right] \\
& \mathrm{C}=\left[\begin{array}{llllll}
0.0000 \mathrm{D}+00 & 0.0000 \mathrm{D}+00 & 0.0000 \mathrm{D}+00 & 0.0000 \mathrm{D}+00 & 1.0000 \mathrm{D}+00 & 0.0000 \mathrm{D}+00 \\
0.0000 \mathrm{D}+00 & -.4682 \mathrm{D}-01 & -.1443 \mathrm{D}-03 & 0.0000 \mathrm{D}+00 & -.8228 \mathrm{D}-03 & 0.0000 \mathrm{D}+00
\end{array}\right. \\
& \left.\begin{array}{ll}
0.0000 \mathrm{D}+00 & 0.0000 \mathrm{D}+00 \\
0.7080 \mathrm{D}+00 & 0.6721 \mathrm{D}+01
\end{array}\right] \quad \mathrm{D}=\left[\begin{array}{ll}
0.0000 \mathrm{D}+00 & 0.0000 \mathrm{D}+00 \\
0.0000 \mathrm{D}+00 & 0.0000 \mathrm{D}+00
\end{array}\right]
\end{aligned}
$$

Eigenvalues of matrix $\mathrm{A}\left(\mathrm{sec}^{-1}\right)$ :

$\begin{array}{cccc}\text { Real part } & \text { Imaginary part } & \text { Real part } & \text { Imaginary part } \\ -0.402555 \mathrm{D}+04 & 0.000000 \mathrm{D}+00 & -0.451191 \mathrm{D}+03 & -0.202507 \mathrm{D}+03 \\ -0.158369 \mathrm{D}+04 & 0.000000 \mathrm{D}+00 & -0.254363 \mathrm{D}+02 & 0.000000 \mathrm{D}+00 \\ -0.142605 \mathrm{D}+04 & 0.000000 \mathrm{D}+00 & -0.333333 \mathrm{D}+03 & 0.000000 \mathrm{D}+00 \\ -0.451191 \mathrm{D}+03 & 0.202507 \mathrm{D}+03 & -0.333333 \mathrm{D}+03 & 0.000000 \mathrm{D}+00\end{array}$


System matrices for combustor pressure at $2700 \mathrm{psi}$ :

$$
\begin{aligned}
& \mathrm{A}=\left[\begin{array}{cccccc}
-.1532 \mathrm{D}+02 & -.5684 \mathrm{D}+02 & 0.4304 \mathrm{D}+02 & -.1234 \mathrm{D}+09 & -.4825 \mathrm{D}+02 & 0.2618 \mathrm{D}+09 \\
0.5594 \mathrm{D}+02 & -.5382 \mathrm{D}+03 & -.2000 \mathrm{D}+02 & 0.0000 \mathrm{D}+00 & 0.0000 \mathrm{D}+00 & 0.0000 \mathrm{D}+00 \\
0.1115 \mathrm{D}+02 & 0.9505 \mathrm{D}+03 & -.1450 \mathrm{D}+04 & 0.3844 \mathrm{D}+10 & 0.0000 \mathrm{D}+00 & 0.0000 \mathrm{D}+00 \\
0.0000 \mathrm{D}+00 & 0.8333 \mathrm{D}-03 & -.3105 \mathrm{D}-04 & -.2360 \mathrm{D}+03 & 0.0000 \mathrm{D}+00 & 0.0000 \mathrm{D}+00 \\
0.1780 \mathrm{D}+01 & 0.0000 \mathrm{D}+00 & 0.2200 \mathrm{D}+03 & -.8969 \mathrm{D}+09 & -.4417 \mathrm{D}+04 & 0.3125 \mathrm{D}+11 \\
0.0000 \mathrm{D}+00 & 0.0000 \mathrm{D}+00 & 0.5399 \mathrm{D}-05 & 0.6292 \mathrm{D}+02 & -5270 \mathrm{D}-04 & -.8665 \mathrm{D}+03 \\
0.0000 \mathrm{D}+00 & 0.0000 \mathrm{D}+00 & 0.0000 \mathrm{D}+00 & 0.0000 \mathrm{D}+00 & 0.0000 \mathrm{D}+00 & 0.0000 \mathrm{D}+00 \\
0.0000 \mathrm{D}+00 & 0.0000 \mathrm{D}+00 & 0.0000 \mathrm{D}+00 & 0.0000 \mathrm{D}+00 & 0.0000 \mathrm{D}+00 & 0.0000 \mathrm{D}+00
\end{array}\right. \\
& \left.\begin{array}{ll}
0.0000 \mathrm{D}+00 & 0.0000 \mathrm{D}+00 \\
0.0000 \mathrm{D}+00 & 0.0000 \mathrm{D}+00 \\
0.3576 \mathrm{D}+07 & 0.0000 \mathrm{D}+00 \\
0.8438 \mathrm{D}-01 & 0.0000 \mathrm{D}+00 \\
0.0000 \mathrm{D}+00 & 0.8477 \mathrm{D}+07 \\
0.0000 \mathrm{D}+00 & 0.2000 \mathrm{D}+00 \\
-.3333 \mathrm{D}+03 & 0.0000 \mathrm{D}+00 \\
0.0000 \mathrm{D}+00 & -.3333 \mathrm{D}+03
\end{array}\right] \\
& B=\left[\begin{array}{ll}
0.0000 \mathrm{D}+00 & 0.0000 \mathrm{D}+00 \\
0.0000 \mathrm{D}+00 & 0.0000 \mathrm{D}+00 \\
0.0000 \mathrm{D}+00 & 0.0000 \mathrm{D}+\infty 0 \\
0.0000 \mathrm{D}+00 & 0.0000 \mathrm{D}+00 \\
0.0000 \mathrm{D}+00 & 0.0000 \mathrm{D}+00 \\
0.0000 \mathrm{D}+00 & 0.0000 \mathrm{D}+00 \\
0.3333 \mathrm{D}+03 & 0.0000 \mathrm{D}+00 \\
0.0000 \mathrm{D}+00 & 0.3333 \mathrm{D}+03
\end{array}\right] \\
& \mathrm{C}=\left[\begin{array}{cccccc}
0.0000 \mathrm{D}+00 & 0.0000 \mathrm{D}+00 & 0.0000 \mathrm{D}+00 & 0.0000 \mathrm{D}+00 & 1.0000 \mathrm{D}+00 & 0.0000 \mathrm{D}+00 \\
0.0000 \mathrm{D}+00 & -.5203 \mathrm{D}-01 & -.1124 \mathrm{D}-03 & 0.0000 \mathrm{D}+00 & -.7566 \mathrm{D}-03 & 0.0000 \mathrm{D}+00
\end{array}\right. \\
& \left.\begin{array}{ll}
0.0000 \mathrm{D}+00 & 0.0000 \mathrm{D}+\infty \\
0.8777 \mathrm{D}+\infty & 0.7801 \mathrm{D}+01
\end{array}\right] \quad \mathrm{D}=\left[\begin{array}{ll}
0.0000 \mathrm{D}+\infty 0 & 0.0000 \mathrm{D}+\infty 0 \\
0.0000 \mathrm{D}+00 & 0.0000 \mathrm{D}+\infty 0
\end{array}\right]
\end{aligned}
$$

Eigenvalues of matrix A ( $\left.\sec ^{-1}\right)$ :

$\begin{array}{cccc}\text { Real part } & \text { Imaginary part } & \text { Real part } & \text { Imaginary part } \\ -0.386847 \mathrm{D}+04 & 0.000000 \mathrm{D}+00 & -0.413837 \mathrm{D}+03 & -0.240277 \mathrm{D}+03 \\ -0.141679 \mathrm{D}+04 & 0.000000 \mathrm{D}+00 & -0.196700 \mathrm{D}+02 & 0.000000 \mathrm{D}+00 \\ -0.139069 \mathrm{D}+04 & 0.000000 \mathrm{D}+00 & -0.33333 \mathrm{D}+03 & 0.000000 \mathrm{D}+00 \\ -0.413837 \mathrm{D}+03 & 0.240277 \mathrm{D}+03 & -0.333333 \mathrm{D}+03 & 0.000000 \mathrm{D}+00\end{array}$




\section{APPENDIX D \\ MODELING OF STRUCTURAL DYNAMICS}

One of the critical points where severe damage is likely to occur is assumed to be located at the root of the turbine blades. Since both the damage and subsequent crack initiation sites are confined within a small region of the blade, a linear elastic approach is adopted for macroscopic modeling of the structural dynamics and to predict transient stresses at the point of potential failure. In this approach, the blade geometry, properties of the blade material, and state variables of the plant dynamic model (see Appendix C) are used as inputs to the finite element analysis program to generate a discretized representation of the blade structure and its loading conditions. The resulting stiffness matrix, mass matrix, and force vector are then used to obtain a modal solution for the displacements. In the last step, the stress-displacement relations from the finite-element analysis are used to predict the stresses at the critical point(s) of the blade structure. The general approach is shown in the flow chart of Figure D.1.

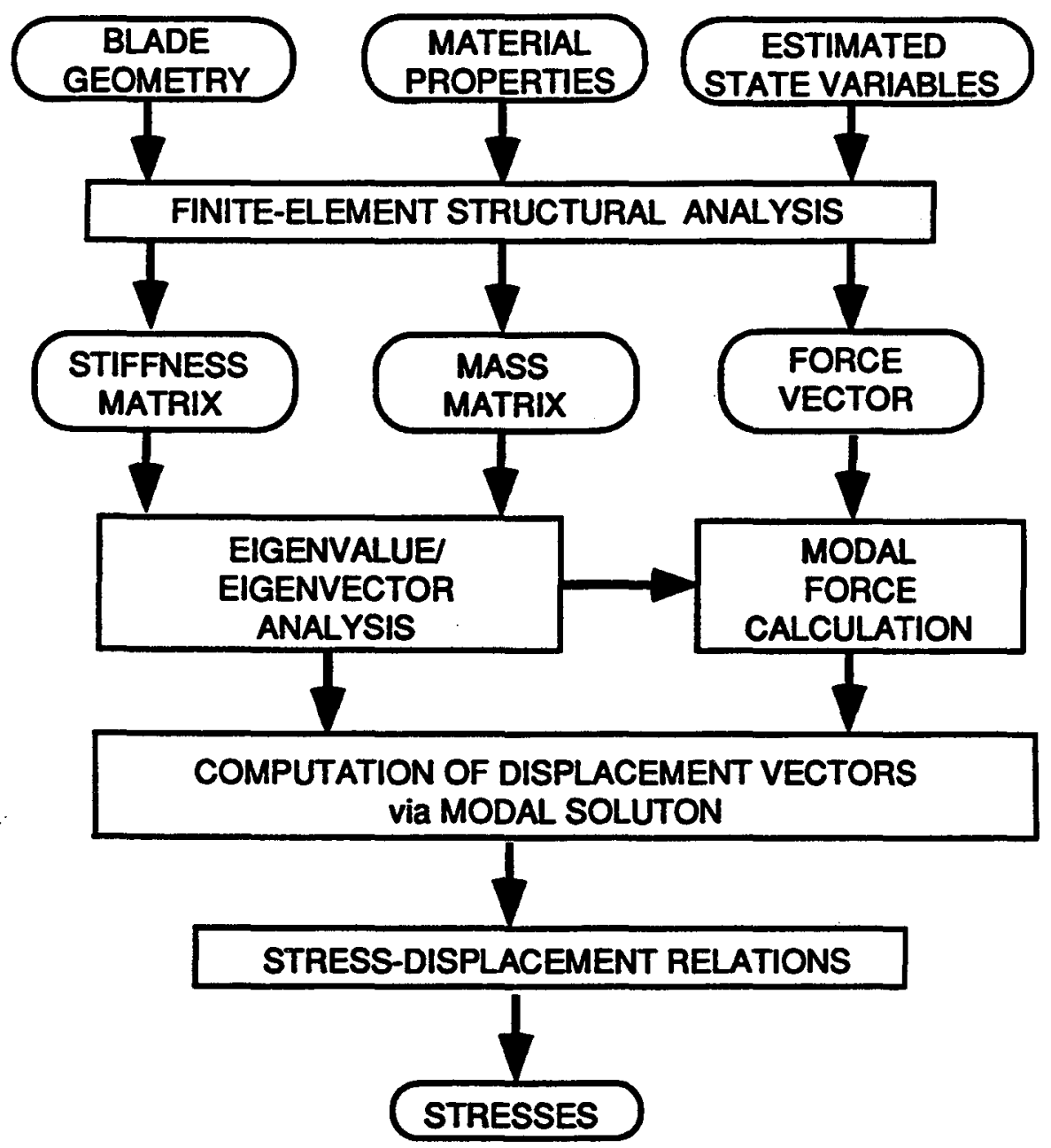

Figure D.1 Flow Chart for the Structural Model of a Turbine Blade 
For simplicity of computation, the turbine blade is approximated to behave like a beam as shown in Figure D.2. Let the torque applied to the turbine shaft at time $t$ be $\tau(t)$. Then, the (time-dependent) mean load, $P_{m}(t)$, per unit length exerted on a typical blade is computed by

$$
P_{m}(t)=\frac{\tau(t)}{N_{B} L r_{m}}
$$

where $N_{B}$ is the number of blades of the turbine, $L$ is the length of the turbine blade and $I_{m}$ is the mean radius of the blade for rotation about the shaft axis. The mean load, $\mathbf{P}_{\mathbf{m}}(t)$, serves as the static load on the blade at time $t$. The dynamic load is assumed to oscillate with an amplitude proportional to the static load and a frequency equal to the product of the shaft speed, $\Omega(t)$, and the number, $N v$, of stationary vanes. The total load per unit length acting on one turbine blade at time $t$ is then given as the sum of the static and dynamic loads per unit length as:

$$
P(t)=P_{m}(t)+P_{A}(t) \sin \left(\Omega(t) N_{v} t\right)
$$

where $P_{A}(t)=\beta P_{m}(t)$ and the proportionality constant $\beta$ is assumed to be an approximate representation of steady-state fluid dynamics within the turbine.

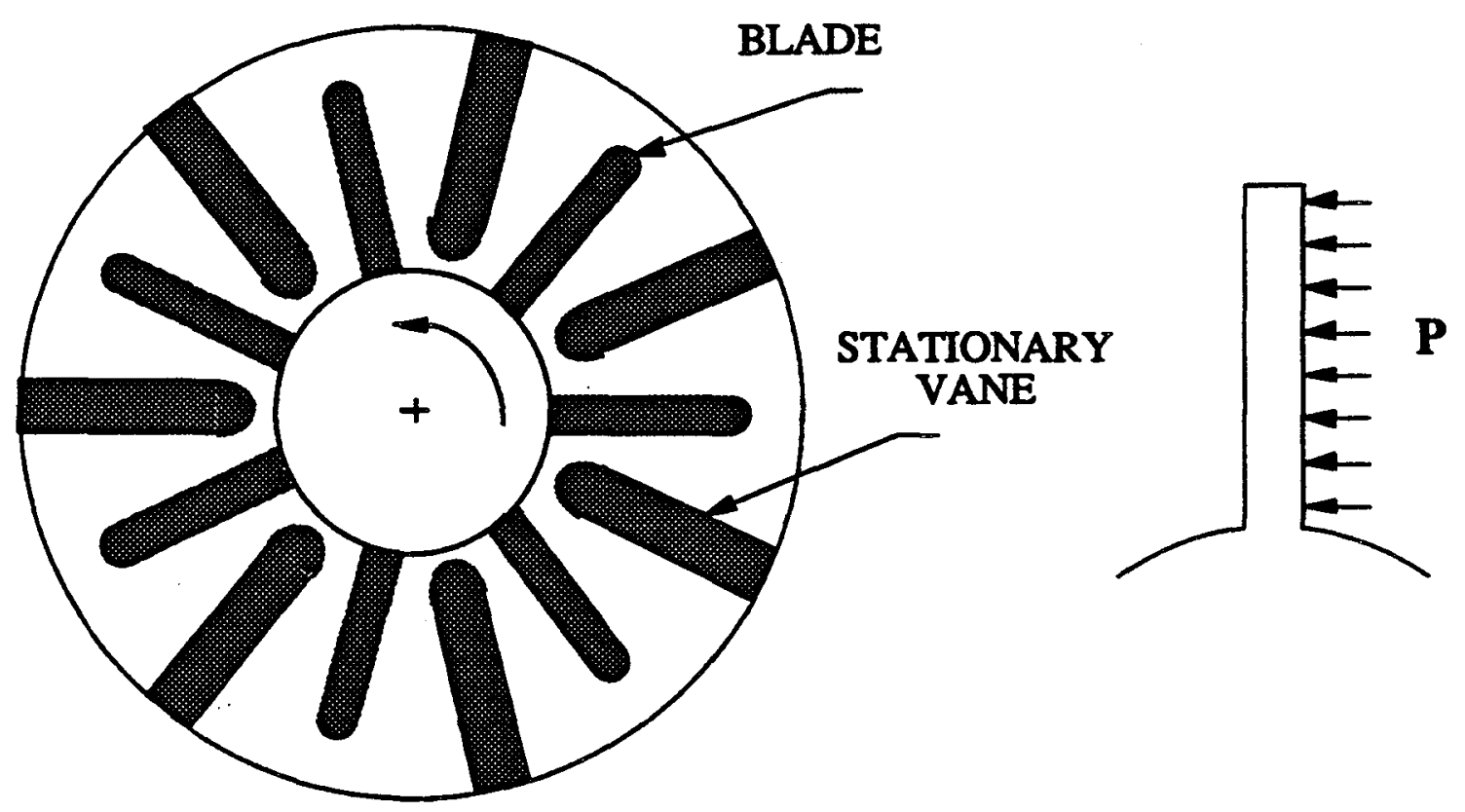

Figure D.2 Loading of a Turbine Blade

With the total load per unit length given by eq. (D.2), the finite element method is used to estimate the stress and strain at the root of the turbine blade. The blade is modeled to consist of two beam elements with three nodes equally spaced as shown in Figure D.3. The model has a total of eighteen degrees of freedom where each node has six degrees of freedom with three translational and three rotational modes. The first node is fixed and thereby all six degrees of freedom associated with the first node are 
suppressed. This is achieved via the penalty approach [Chandrupatla (1991)] in which the spring stiffness at each of the six degrees of freedom at the first node is set to a very large value. In contrast, the use of the elimination approach [Chandrupatla (1991)] would reduce the order of the model to twelve. Ease of implementation in the existing finite-element analysis code is the rationale for using the penalty approach as opposed to the elimination approach.

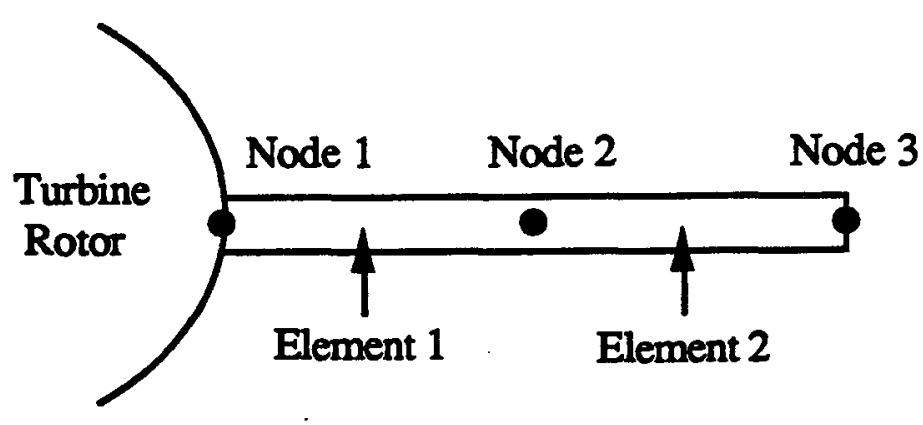

Figure D.3 Finite Element Model of a Turbine Blade

Let a represent the nodal displacements of a beam element, where a is a $12 \times 1$ vector with 6 degrees of freedom at each node, the displacement field $u(x)$ along the element can be expressed in terms of the nodal displacements a and an interpolation matrix N:

$$
\mathbf{u}=\mathbf{N} \mathbf{a}
$$

where $u(x)$ contains three translational and three rotational displacements at location $x$ of the beam element, and each element of the matrix $\mathbf{N}$ is a polynomial interpolation function of $x$. The dimension of $u$ is $6 \times 1$, and the dimension of $N$ is $6 \times 12$. The generalized strain field $\varepsilon$ is then obtained via differentiation of the displacement field as:

$$
\varepsilon=\mathbf{L} \mathbf{u}=\mathbf{L} \mathbf{N} \mathbf{a}=\mathbf{B} \mathbf{a}
$$

where $L$ is a differential operator and $B=L N$. The dimensions of $L$ and $B$ are $4 \times 6$ and $4 \times 12$, respectively. The generalized strain vector $\varepsilon$ contains 4 elements representing the generalized strains due to twisting, stretching and bending in two directions orthogonal to the beam axis. The twisting action produces shear stresses. The stretching and bending actions produce tensile or compressive stresses. For linear elastic materials, the generalized stress vector $\sigma$ is related to the generalized strain vector $\varepsilon$ by a matrix D such that

$$
\sigma=D \varepsilon
$$

The next step is to model the beam dynamics with nodal displacements as the variables of motion. From eq. (D.3), the total kinetic energy, T, is computed by integrating the kinetic energy of the mass, pdx, of the differential element along the entire length, $l$, of the element : 


$$
\begin{aligned}
\mathbf{T} & =\frac{1}{2} \int_{0}^{l} \dot{\mathbf{u}}^{\mathbf{T}} \rho \dot{\mathbf{u}} \mathrm{dx} \\
& =\frac{1}{2} \int_{0}^{l} \dot{\mathbf{a}}^{\mathbf{T}} \mathbf{N}^{\mathbf{T}} \rho \mathbf{N a \dot { a }} \mathrm{dx} \\
& =\frac{1}{2} \dot{\mathbf{a}}^{\mathbf{T}}\left(\int_{0}^{l} \mathbf{N}^{\mathbf{T}} \rho \mathbf{N} \mathrm{dx}\right) \dot{\mathbf{a}} \\
& =\frac{1}{2} \dot{\mathbf{a}}^{\mathbf{T}} \mathbf{M} \dot{\mathbf{a}}
\end{aligned}
$$

where $p$ is the diagonal matrix containing the mass density terms and $\mathbf{M}=$ $\int_{0}^{l} \mathbf{N}^{\mathbf{T}} \rho \mathbf{N} \mathrm{dx}$ is generally called the mass matrix of the element. The potential energy, $\mathrm{V}$, can also be computed by integrating the strain energy of the element as given below:

$$
\begin{aligned}
\mathbf{V} & =\frac{1}{2} \int_{0}^{l} \varepsilon^{\mathrm{T}} \sigma \mathrm{dx} \\
& =\frac{1}{2} \int_{0}^{l} \varepsilon^{\mathrm{T}} \mathbf{D} \varepsilon \mathrm{dx} \\
& =\frac{1}{2} \int_{0}^{l} \mathbf{a}^{\mathrm{T}} \mathbf{B}^{\mathrm{T}} \mathbf{D B a} \mathrm{dx} \\
& =\frac{1}{2} \mathbf{a}^{\mathrm{T}}\left(\int_{0}^{l} \mathbf{B}^{\mathrm{T}} \mathbf{D B} \mathrm{dx}\right) \mathbf{a} \\
& =\frac{1}{2} \mathbf{a}^{\mathrm{T}} \mathbf{K} \mathbf{a}
\end{aligned}
$$

where $\mathrm{K}=\int_{0}^{l} \mathrm{~B}^{\mathrm{T}} \mathrm{DB} \mathrm{dx}$ is the stiffness matrix. Since the energy is additive, the mass and stiffness matrices of the whole structure consisting of the finite element meshes can be obtained by the principle of superposition. The matrices of the individual elements are added together at each degree of freedom in the formulation of matrices for the entire structure. The matrices $M$ and $K$ are both real symmetric since $p$ and $D$ are symmetric. Furthermore, the mass matrix $M$ is positive definite because the kinetic energy is always positive with a nonzero velocity, $\dot{a}$. The stiffness matrix $K$ is positive semidefinite due to nonnegative strain energy. From the Lagrange equations of motion, the beam dynamics in the absence of any damping is derived below:

$$
\begin{aligned}
& \frac{d}{d t}\left(\frac{\partial(T-V)}{\partial \dot{a}}\right)-\frac{\partial(T-V)}{\partial \mathbf{a}}=\mathbf{f} \\
\Rightarrow & \mathbf{M} \ddot{\mathbf{a}}+\mathbf{K} \mathbf{a}=\mathbf{f}
\end{aligned}
$$

where $f$ is the vector of the concentrated nodal forces applied to the degrees of freedom. If the distributed load, $p$, is applied, the work done by $\mathbf{p}$ along the length of the beam 
should be equal to the work done by the equivalent concentrated forces, $f$, on the nodal displacements such that

$$
\mathbf{a}^{\mathrm{T}} \mathbf{f}=\int_{0}^{l} \mathbf{u}^{\mathrm{T}} \mathrm{pdx}=\int_{0}^{l} \mathbf{a}^{\mathrm{T}} \mathrm{N}^{\mathrm{T}} \mathrm{p} \mathrm{dx}=\mathbf{a}^{\mathrm{T}} \int_{0}^{l} \mathbf{N}^{\mathrm{T}} \mathrm{p} \mathrm{dx}
$$

Since eq. (D.9) is true for all nodal displacements a, the equivalent forces can be calculated as:

$$
\mathbf{f}=\int_{0}^{l} \mathbf{N}^{\mathrm{T}} \mathbf{p} \mathrm{dx}
$$

Eq. (D.8) does not include the forces caused by damping. If the damping effects need to be considered, the damping coefficients can be approximated as a linear combination of the mass and stiffness matrices [Weaver (1991)] such that $\mathbf{C}=\alpha \mathbf{M}+$ BK. Then, eq. (D.8) is modified as

$$
M \ddot{a}+(\alpha M+\beta K) \dot{a}+K a=\mathbf{f}
$$

Since eq. (D.11) contains a set of coupled differential equations, its numerical solution through direct integration is time-consuming. An alternative approach is to find a transformation matrix and diagonalize the coupled equations into a set of decoupled equations that have the same form as ordinary second order differential equations. This set of decoupled equations can be easily solved and transformed back to the original nodal displacements. If the nodal forces, $f$, are in the form of general periodic functions, then they can also be expanded in Fourier series containing trigonometric functions such that the exact solution can be derived. Another advantage of this approach is that some of the decoupled equations associated with high natural frequencies lead to small amplitudes of vibrations and may therefore be neglected in computation. Consequently, computation time is saved without any significant loss of accuracy.

To decouple eq. (D.11), first consider the following eigenvalue problem:

$$
\mathbf{K x}=\omega^{2} \mathbf{M x}
$$

Since eqs. (D.6) and (D.7) yield the symmetric positive definite mass matrix $M$ and the symmetric positive semidefinite stiffness matrix $K$, the eigenvalues associated with eq. (D.12) are all real and nonnegative. Let $\omega_{1}^{2}, \omega_{2}^{2}, \ldots, \omega_{n}^{2}$ be the eigenvalues of eq. (D.12) and $x_{1}, x_{2}, \cdots, x_{n}$ be the corresponding eigenvectors, where $\omega_{i}$ and $x_{i}$ are generally referred to the natural frequency and mode shape associated with the $i^{\text {th }}$ mode of the structure [Weaver (1991)]. For any two distinct eigenvalues, $\omega_{j}^{2}$ and $\omega_{j}^{2}$, eq. (D.12) can be written as

$$
\begin{aligned}
& K \mathbf{x}_{\mathbf{i}}=\omega_{\mathbf{i}}^{2} \mathbf{M} \mathbf{x}_{\mathbf{i}} \\
& \mathbf{K} \mathbf{x}_{\mathbf{j}}=\omega_{j}^{2} \mathbf{M} \mathbf{x}_{\mathbf{j}}
\end{aligned}
$$


Premultiplying both sides of eq. (D.13a) by $\mathbf{x}_{\mathbf{j}}{ }^{\mathrm{T}}$ and eq. (D.13b) by $\mathbf{x}_{\mathbf{i}}^{\mathrm{T}}$, it becomes:

$$
\begin{aligned}
& x_{j}{ }^{T} K x_{i}=\omega_{i}^{2} x_{j}{ }^{T} M x_{i} \\
& x_{i}{ }^{T} K x_{j}=\omega_{j}^{2} x_{i}{ }^{T} M x_{j}
\end{aligned}
$$

Since $M$ and $K$ are both symmetric, $\mathbf{x}_{\mathbf{j}}^{\mathrm{T}} \mathbf{K} \mathbf{x}_{\mathbf{i}}=\mathbf{x}_{\mathbf{i}}{ }^{\mathrm{T}} \mathbf{K} \mathbf{x}_{\mathbf{j}}$ and $\mathbf{x}_{\mathbf{j}}^{\mathrm{T}} \mathbf{M} \mathbf{x}_{\mathbf{i}}=\mathbf{x}_{\mathbf{i}}{ }^{\mathrm{T}} \mathbf{M} \mathbf{x}_{\mathbf{j}}$. Subtracting (D.14b) from (D.14a) results in

$$
0=\left(\omega_{i}^{2}-\omega_{j}^{2}\right) \mathbf{x}_{j}^{T} \mathbf{M} \mathbf{x}_{\mathbf{i}}
$$

If $\omega_{i}^{2} \neq \omega_{j}^{2}$, then both $\mathbf{x}_{j}{ }^{T} \mathbf{M x} x_{i}$ and $\mathbf{x}_{j}{ }^{T} \mathbf{K x} x_{i}$ must be equal to zero. This result leads to the following important property: if all the eigenvalues are distinct, the eigenvectors are mutually orthogonal with respect to the mass and stiffness matrices. In fact, if an eigenvalue $\lambda_{i}$ for real symmetric matrices $M$ and $K$ has multiplicity $m$, then there exist exactly $m$ linearly independent eigenvectors corresponding to these $m$ eigenvalues [Meirovitch (1980)]. It is always possible to choose such combinations that these eigenvectors are mutually orthogonal and orthogonal to eigenvectors belonging to the remaining eigenvalues. Let each eigenvector, $x_{i}$, be normalized with respect to the mass matrix $M$, i.e., $x_{i} \mathrm{~T}^{\mathrm{T}} \mathrm{x}_{\mathrm{i}}=1$ for $\mathrm{i}=1,2, \ldots \mathrm{n}$. Then, following either eq. (D.14a) or (D.14b), it can be deduced that $\mathbf{x}_{\mathbf{i}}{ }^{\mathbf{T}} \mathbf{K} \mathbf{x}_{\mathbf{i}}=\omega_{i}^{2}$. The above results can be written as:

$$
\begin{array}{lll}
\mathbf{x}_{\mathbf{i}}^{\mathrm{T}} \mathbf{M} \mathbf{x}_{\mathbf{j}}=0 & \text { if } \mathrm{i} \neq \mathrm{j} & \mathbf{x}_{\mathbf{i}}^{\mathrm{T}} \mathbf{M} \mathbf{x}_{\mathbf{j}}=1 \quad \text { if } \mathrm{i}=\mathbf{j} \\
\mathbf{x}_{\mathbf{i}}^{\mathrm{T}} \mathbf{K} \mathbf{x}_{\mathbf{j}}=0 & \text { if } \mathrm{i} \neq \mathbf{j} & \mathbf{x}_{\mathbf{i}}^{\mathrm{T}} \mathbf{K} \mathbf{x}_{\mathbf{j}}=\omega_{i}^{2} \quad \text { if } \mathrm{i}=\mathbf{j}
\end{array}
$$

Let $\Psi=\left[\begin{array}{llll}x_{1} & x_{2} & \cdots & x_{n}\end{array}\right]$ and $\Lambda=\left[\begin{array}{cccc}\omega_{1}^{2} & 0 & \cdots & 0 \\ 0 & \omega_{2}^{2} & & \vdots \\ \vdots & & \ddots & \vdots \\ 0 & \cdots & \cdots & \omega_{n}^{2}\end{array}\right]$. Then, eqs (D.16a) and (D.16b) can be expressed as

$$
\Psi \mathrm{T} M \Psi=\mathbf{I} \quad \text { and } \quad \Psi \mathrm{T} \mathbf{K} \Psi=\mathbf{\Lambda}
$$

Then, with premultiplication by $\Psi$ T, eq. (D.11) can be decoupled as:

$$
\ddot{q}+(\alpha I+\beta \Lambda) \dot{q}+\Lambda q=\Psi^{T_{\mathbf{f}}}
$$

where $q=\Psi^{-1} \mathrm{a}$ and $\Psi-1$ exists because of linear independence of eigenvectors, $x_{i}, i=1,2, \ldots n$. After solving eq. (D.18) for the vector $q$, the nodal displacement vector can be computed from the transformation $\mathbf{a}=\Psi \mathrm{q}$. The generalized strains and stresses at the selected points are then recovered from eqs. (D.4) and (D.5). 
Due to the damping effects, the individual components of the displacement vector, a, may have different phase angles. It is expensive to compute the stress at a certain point by simple superposition of the effects of twisting, stretching and bending. It is assumed in the simulation that the damping effects are negligible and all components of the displacement vector have an identical phase angle. The stresses caused by stretching and bending are added together for the normal stress. The shear stress is obtained from twisting. The maximum principal stress is an approximate representation of the stress at the corners of the root of a turbine blade, which is used to estimate the fatigue strain-induced damage rate. 


\section{APPENDIX E}

\section{MODELING OF INTEGRATED FATIGUE-CORROSION-CREEP DAMAGE}

Experimental results indicate that, in gas turbine materials such as Ni-base superalloys, reduction of fatigue life at elevated temperatures (as low as $30 \%$ of the melting point) is significantly influenced by the gaseous environment [Courtney (1990); Suresh (1991)]. The contributions of creep and corrosion on structural damage become significant at elevated temperatures due to the effects on micromechanisms of fatigue fracture. For example, the fatigue failure at a low temperature usually results from transgranular fracture but it may occur due to intergranular fracture at an elevated temperature. The impact of elevated temperatures on damage could be both beneficial and detrimental. Beneficial effects include a dispersal of the slip, and the detrimental effects are usually caused by creep and corrosion. In fact, the fatigue life at a high temperature may increase with an increase in the frequency of strain reversals [Suresh (1991)]. While the fatigue damage is cycle-dependent, the creep damage and corrosion damage are time-dependent.

Although the time-dependent part of the fatigue-creep damage is thermally activated and results from both creep and corrosion, linear elastic fracture mechanics can be used for damage prediction provided that the stress intensity factor range remains the controlling parameter for crack propagation. This is valid if the zone of inelastic deformation at the crack tip is small in size compared to the uncracked length. However, at very high temperatures and low cycles, the elastic fracture mechanism may not be prevalent as the inelastic zone becomes appreciably large. Furthermore, the material properties such as the tensile strength and fracture toughness change significantly at elevated temperatures relative to their nominal values. The dynamics of creep damage may be modeled from these perspectives. The total strain rate $\dot{\varepsilon}$ consisting of elastic strain rate $\dot{\varepsilon}_{e}$ and plastic strain rate $\dot{\varepsilon}_{p}$ is modeled for an elasticnonlinear viscous material under uniaxial tension in terms of the instantaneous stress $\sigma$ and the stress rate $\dot{\sigma}$ as [Lemaitre (1992)]:

$$
\dot{\varepsilon}=\dot{\varepsilon}_{\mathrm{e}}+\dot{\varepsilon}_{\mathrm{p}} \text { where } \dot{\varepsilon}_{\mathrm{e}}=\frac{\dot{\sigma}}{\mathrm{E}} \text { and } \dot{\varepsilon}_{\mathrm{p}}=\left(\frac{\sigma}{\sigma_{\mathrm{y}}}\right)^{\mathrm{n}_{\mathrm{c}}}
$$

where the material parameters are: Young's modulus $E$; yield strength $\sigma_{y}$; reference creep strain rate $\dot{\varepsilon}_{\mathbf{y}}$; and power law creep exponent $\mathbf{n}_{\mathbf{c}}$. Eq. (6) may have to be modified for multi-axial stress conditions in the critical components. In that case, the effective stress needs to be defined as a function of the stress tensor.

Some investigators, for example [Saxena (1988)] and [Zamrik and Davis (1990)], have computed the total damage as the sum of corrosion-fatigue and creep damages that are obtained independently. That is, the cumulative damage $D_{c f}$ in creepfatigue cycling has been defined upon completion of $n$ cycles as:

$$
D_{c f}=D_{f}+D_{c}
$$


where $D_{c}$ is the creep damage part; $D_{f}$ is the fatigue damage part; and $D_{c f}$ is normalized to unity at the onset of failure. Similar to the linear damage rule for cumulative fatigue damage, the creep damage is often based on time summation as:

$$
D_{c}=\sum_{i=1}^{n}\left\{N_{f h} \int_{0}^{t_{h}} \frac{d t}{t_{r}}\right\}_{i}
$$

where $\mathrm{N}_{\mathrm{fh}}$ is the number of cycles to failure in creep-fatigue; tr is the time to creeprupture; and th is the hold time. However, the above time summation model lacks the input of the material properties. Based on observed failure modes for the creep-fatigue interaction, ductility is considered to have a major influence on failure of engineering materials. Zamrik and Davis (1990) have proposed a model of the creep damage part $D_{c}$ based on the ductility exhaustion concept as follows:

$$
D_{c}=\sum_{i=1}^{n}\left\{N_{t h} \int_{0}^{t_{h}}\left[\frac{\Delta \dot{\varepsilon}_{c p}}{\vartheta\left(\Delta \dot{\varepsilon}_{c p}\right)}\right] d t\right\}_{i}
$$

where $\Delta \dot{\varepsilon}_{\mathrm{cp}}$ is the rate of creep strain range during the hold-time period, and $\vartheta\left(\Delta \dot{\varepsilon}_{\mathrm{cp}}\right)$ is the material creep ductility as a function of $\Delta \dot{\varepsilon}_{\text {cp }}$. If the creep strain accumulation during the hold-time period is assumed to obey the power law, then

$$
\Delta \dot{\varepsilon}_{c p}=A(\Delta \sigma)^{q} t^{r-1}
$$

where $A, r$ and $q$ are constants, and $\Delta \sigma$ is the stress range at time $t$ during hold period. Pineau (1989) also proposed a ductility exhaustion model where the creep damage $D_{c}$ is expressed in terms of the instantaneous values of plastic strain rate $\dot{\varepsilon}_{p}$, stress $\sigma$, and temperature $T$ as:

$$
D_{c}(t)=\int_{0}^{t}\left(\frac{\dot{\varepsilon}_{p}(\sigma(\tau), T(\tau))}{\dot{\varepsilon}_{s}(\sigma(\tau), T(\tau))}\right) \times \frac{d \tau}{t_{r}(\sigma(\tau), T(\tau))}
$$

where $t$ is the current time, and $t_{r}$ is the total time to rupture due to creep, and $\dot{\varepsilon}_{s}$ is the stationary creep rate [Coutsouradis et al. (1978)]. The two models in eqs. (E.5) and (E.6) in view of the fact that ductility is the primary parameter for creep damage at elevated temperatures.

Modeling of corrosion-fatigue crack growth in gaseous environments has been reported by several investigators including Wei (1989). The governing equations for damage dynamics are formulated on the basis of mass balance and reaction kinetics where the state variables are gas pressure at the crack tip and percent of surface reactions. In general, the corrosion-fatigue crack growth rate (da/dN) cf can be obtained as a modification of the pure fatigue growth rate $(\mathrm{da} / \mathrm{dN})_{\mathrm{f}}$ by including the states of gas 
pressure and surface reactions. If the dynamics of corrosion are likely to be slow relative to fatigue dynamics except for operations at elevated temperatures. Depending on the operating conditions, it might be possible to eliminate the above state variables by varying the parameters of the fatigue crack growth equation slowly with time. Therefore, the constraint parameters $\beta$ and $\Gamma$ in eqs. (2.4) and (2.5) may also be chosen as slowly varying functions of time to incorporate the effects of corrosion.

Following the procedures described in Section 4, the cycle-dependent corrosionfatigue crack growth rate $(\mathrm{da} / \mathrm{dN})_{\mathrm{cf}}$ can be converted to the time-dependent crack growth rate $(\mathrm{da} / \mathrm{dt})_{\mathrm{cf}}$. The corrosion-fatigue damage is defined, similar to eq. 4.31 , as $D_{c f}=a / a^{*}$ where $a^{*}$ is the critical crack length. Then, following the structure of eq. (2.2), a dynamic model of the combined effects of creep and fatigue damage at a single critical point is proposed as follows:

$$
\begin{array}{ll}
\frac{d D_{c f}}{d t}=h_{c f}\left(D_{c f}(t), D_{c r}(t), q(x, t), t\right) ; & D_{c f}\left(t_{0}\right)=D_{c f 0} \quad h_{c f} \geq 0 \quad \forall t \\
\frac{d D_{c}}{d t}=h_{c r}\left(D_{c f}(t), D_{c r}(t), q(x, t), t\right) ; & D_{c r}\left(t_{0}\right)=D_{c r 0} \quad h_{c r} \geq 0 \quad \forall t
\end{array}
$$

In the above equation set, the damage vector is expressed as $v(t)=\left[D_{c f} D_{c}\right]^{T}$. For multiple critical points, the damage vector includes the damage components at all points. Finally, a scalar measure $D$ of total damage is expressed as a function of the corrosion-fatigue damage and creep damage, and the end of service life occurs at $D=1$. 


\section{REFERENCES}

Bannantine, J. A., Comer, J. J. and Handrock, J. L., 1990, Fundamentals of Metal Fatigue Analysis , Prentice Hall.

Blackburn, J. F., Reethof, G. and Shearer, J. L., 1960, Fluid Power Control, The M.I.T. Press.

Boller, C. and Seeger, T., 1987, Materials Data for Cyclic Loading, Elsevier.

Bolotin, V. V., 1989, Prediction of Service Life for Machines and Structures, ASME.

Chandrupatla, T. R. and Belegundu, A. D., 1991, Introduction to Finite Elements in Engineering, Prentice-Hall, NJ.

Coutsouradis, D. et al., eds., 1978, High Temperature Alloys for Gas Turbines, Applied Science, London, p. 513.

Dowling, N. E., 1983, "Fatigue Life Prediction for Complex Load Versus Time Histories," Journal of Engineering Materials and Technology, Trans. ASME, Vol. 105, pp. 206-214.

Doyle, J. C., Glover, K., Khargonekar, P. and Francis, B., 1989, "State-Space Solutions to Standard $\mathrm{H}_{2}$ and $\mathrm{H}_{\infty}$ Control Problems," IEEE Transactions on Automatic Control, Vol. 34, No. 8, Aug., pp.831-847.

Dugdale, D. S., 1960, "Yielding of Steel Sheets Containing Slits," Journal of Mechanics, Physics, and Solids, Vol. 8, pp. 100-104.

Duyar, A., Eldem, V., Merrill, W.C. and Guo,.T-H., 1991, "State Space Representation of the Open-Loop Dynamics of the Space Shuttle Main Engine," ASME Journal of Dynamic Systems, Measurement, and Control, Vol. 113, December, pp. 684-690.

Fuchs, H. O. and Stephens, R. I., 1980, Metal Fatigue in Engineering, John Wiley \& Sons.

Gill, P.E., Murray, W., Saunders, M.A. and Wright, M.H., 1991, NPSOL Version 4.06, Software Distribution Center, Stanford University, Palo Alto, CA.

Goodwin, G.C. and Sin, K.S., 1984, Adaptive Filtering Prediction and Control, Englewood Cliffs.

Hertzberg, R.W., 1989, Deformation and Fracture Mechanics of Engineering Materials, John Wiley, New York

Hicks, T. G. and Edwards, T. W., 1971, Pump Application Engeering, McGraw-Hill, New York.

IMSL Manual, Math/Library, Vol. 3, pp.895-902.

Kidd, P.T., 1991, "Modification of Adaptive Control Laws Using Adaptive Gain Feedback," International Journal of Control, Vol. 53, pp. 145-162.

Lorenzo, C.F. and Merill, W.C., 1991a, "An Intelligent Control System for Rocket Engines: Need, Vision and Issues," IEEE Control Systems Magazine, Vol. 11, No. 1, June, pp. $42-46$.

Lorenzo, C.F. and Merill, W.C., 1991b, "Life Extending Control: A Concept Paper," American Control Conference, Boston, MA, June, pp. 1080-1095.

Luenberger, D.G., 1984, Linear and Nonlinear Programming, Addison-Wesley, Menlo Park, CA. 
Manson, S. S. and Halford, G. R., 1981, "Practical Implementation of the Double Linear Damage Rule and Damage Curve Approach for Treating Cumulative Fatigue Damage," Int. Journal of Fracture, Vol. 17, No.2, pp. 169-192.

Marco, S. M. and Starkey, W. L., 1954, " A Concept of Fatigue Damage," Trans. ASME, Vol. 76, No.4, pp. 627-632.

Maybeck, P. S., 1979, Stochastic Models, Estimation, and Control, Academic Press.

Meirovitch, L., 1980, Computational Methods in Structural Dynamics, Sijthoff \& Noordhoff.

Miner, M. A., 1945, "Cumulative Damage in Fatigue," Journal of Applied Mechanics, Trans. ASME, Vol. 67, Sep., pp.A159-A164.

NASA Lewis Research Center, 1991, Unpublished data on Modeling of the Space Shuttle Main Engine, Cleveland, $\mathrm{OH}$.

Newman, J. C., Jr., 1981, "A Crack Closure Model for Predicting Fatigue Crack Growth under Aircraft Spectrum Loading," Methods and Models for Predicting Fatigue Crack Growth under Random Loading. ASTM STP 748, pp. 53-84.

Newman, J. C., Jr., 1984, "A Crack Opening Stress Equation for Fatigue Crack Growth," Int. Journal of Fracture, Vol. 24, pp. R131-R135.

Newman, J. C., Jr., Phillips, E. P., Swain, M. H. and Everett, Jr., R. A., 1992, "Fatigue Mechanics : An Assessment of A Unified Approach to Life Prediction," ASTM Symposium On Advances in Fatigue Lifetime Predictive Techniques, ASTM STP 1122, M.R. Mitchell and R.W. Landgraf, Eds., American Society for Testing Materials, Philadelphia, pp. 5-27.

Noll, T., Austin, E., Donley, S., Graham, G., Harris, T., Kaynes, I., Lee, B. and Sparrow, J., 1991, "Impact of Active Controls Technology on Structural Integrity," 32nd AIAA/ASME/ASCE/AHSIASC Structures, Structural Dynamics, and Materials Conference, Baltimore, MD, April, pp. 1869-1878.

Packard, A., Doyle, J. and Balas, G., 1993, "Linear, Multivariable Robust Control with a m Perspective, " Journal of Dynamic Systems, Measurement, and Control, Transactions of the ASME, 50th Anniversary Issue, Vol. 115, June, pp.428-438.

Paris, P. C. and Erdogan, F., 1963, "A Critical Analysis of Crack Propagation Laws," Journal of Basic Engineering, Trans. ASME, Vol. D85, pp.528-534

Pineau, A., 1989, "Elevated Temperature Life Prediction Methods," Advances in Fatigue in Science and Technology, eds. C.M. Branco and L.G. Rosa, Proceedings of NATO ASI Series, Kluwer Academic, Dordrecht, pp. 313-338.

Ray, A., 1976, "Mathematical Modeling and Digital Simulation of a Commercial Scale High Temperature Gas-Cooled Reator (HTGR) Steam Power Plant," Ph. D. Thesis, Northeastern University, Boston, MA.

Ray, A., 1990, "Multi-Level Hypotheses Testing for Fault Detection in Continuous Prosess," Journal of Dynamic Systems, Measurement, and Control, Transactions of the ASME, Vol. 112, Dec., pp.787-790

Ray, A., Berkowitz, D. A. and Sumaria, V. H., 1980, "Nonlinear Dynamic Model of a Fluidized-Bed Steam Generation System," Journal of Engineering for Power. Trans. ASME, Vol. 102, Jan., pp.202208.

Ray, A., Wu, M-K., Carpino, M., and Lorenzo, C.F., 1993a, "Damage-Mitigating Control of Mechanical Systems: Part I - Conceptual Development and Model Formulation," American Control Conference, San 
Francisco, pp. 1731-1735. (An expanded version of this paper will appear in ASME Journal of Dynamic Systems, Measurement, and Control.)

Ray, A., Wu, M-K., Carpino, M., and Lorenzo, C.F., 1993b, "Damage-Mitigating Control of Mechanical Systems: Part II - Formulation of an Optimal Policy and Simulation," American Control Conference., pp. 3146-3150. (An expanded version of this paper will appear in ASME Journal of Dynamic Systems, Measurement, and Control.)

Royden, H.L., 1988, Real Analysis, 3rd edition, Macmillan Publishing Company, New York.

Saxena, A., 1988, "A Model for predicting the effect of frequency on fatigue crack growth at elevated temperature," Fatigue of Engineering Materials and Structures, Vol. 3, pp. 247-255.

Shames, I. H., 1962, Mechanics of Fluids, McGraw-Hill, New York.

Skogestad, S., Morari, M. and Doyle, J.C., 1988, "Robust Control Of Il-Conditioned Plants: High Purity Distillation," IEEE Transactions on Automatic Control, Vol. 33, No. 12, December, pp. 1092-1105.

Sobczyk, K. and Spencer, B.F., Jr., 1992, Random Fatigue: Data to Theory, Academic Press, Boston, MA.

Stein, G. and Athans, M.A., 1987, "The LQG/LTR Procedure for Multivariable Feedback Control Design," IEEE Transactions on Automatic Control, Vol. AC-32, No. 2, February, pp. 105-114.

Stein, G. and Doyle, J.C., 1991, "Beyond Singular Values and Loop Shapes," IAIAA Journal of Guidance, Control and Dynamics, Vol. 14, No. 2, February, pp. 105-114.

Stengel, RF., 1991, "Intelligent Failure-Tolerant Control," IEEE Control Systems Magazine, Special Issue on Intelligent Control, Vol. 11, No. 1, January-February, pp. 5-14.

Suresh, S., 1991, Fatigue of Materials, Cambridge University Press, Cambridge, U.K.

Sutton, G.P., 1992, Rocket Propulsion Elements, Wiley Interscience, New York.

Swain, M. H., Everett, R. A., Newman, J. C., Jr., and Phillips, E. P., 1990, "The Growth of Short Cracks in 4340 Steel and Aluminum-Lithium 2090," AGARD Report, No. 767, pp. 7.1-7.30.

Tucker, L., and Bussa, S., 1977, "The SAE Cumulative Fatigue Damage Test Program," Fatigue Under Complex Loading: Analyses and Experiments, Vol. AE-6, pp.1-54.

Tucker, L., Landgraf, R., and Brose, W., 1974, "Proposed Technical Report on Fatigue Properties for the SAE handbook," SAE Automotive Engineering Conference, Paper 740279, Detroit, Mi, Feb.

Vidyasagar, M., 1992, Nonlinear Systems Analysis, 2nd ed., Prentice Hall, Englewood Cliffs, NJ.

Weaver, W., Jr. and Johnson, P. R., 1987, Structural Dynamics by Finite Elements, Prentice-Hall, NJ.

Wei, R.P., 1989, "Environmentally Assisted Fatigue Crack Growth," Advances in Fatigue in Science and Technology, ed. C.M. Branco and L.G. Rosa, Proceedings of NATO ASI Series, Kluwer Academic, Dordrecht, Germany.

Zamrik, S.Y. and Davis, D.C., 1990, "Creep-Fatigue Damage Mechanisms and Life Assessment of Two Materials: Type 316 Stainless Steel and Waspalloy," ASM International Conference on Life Assessment and Repar Technology for Combustion Turbine Hot Section Components, April 17-19, Phoenix, AZ.

Zemansky, M. W., Van Ness, H. C., 1966, Basic Engineering Thermodynamics, Mcgraw-Hill, New Youk. 
Public reporting burden for this collection of information is estimated to average 1 hour per response, including the time for reviewing instructions, searching existing data sources, gathering and maintaining the data needed, and completing and reviewing the collection of information. Send comments regarding this burden estimate or any other aspect of this collection of information including suggestions for reducing this burden, to Washington Headquarters Services, Directorate for Information Operations and Reports, 1215 Jefferson Davis Highway, Suite 1204, Arlington, VA 22202-4302, and to the Office of Management and Budget, Paperwork Reduction Project (070s-0188), Washington, DC 20503.

\begin{tabular}{|l|c|c|}
\hline 1. AGENCY USE ONLY (Leave blank) & $\begin{array}{r}\text { 2. REPORT DATE } \\
\text { March } 1994\end{array}$ & $\begin{array}{r}\text { 3. REPORT TYPE AND DATES COVERED } \\
\text { Final Contractor Report }\end{array}$ \\
\hline
\end{tabular}

\section{TITLE AND SUBTITLE}

Damage-Mitigating Control of Space Propulsion Systems for

High Performance and Extended Life

5. FUNDING NUMBERS

\section{AUTHOR(S)}

Asok Ray and Min-Kuang Wu

\section{PERFORMING ORGANIZATION NAME(S) AND ADDRESS(ES)}

8. PERFORMING ORGANIZATION REPORT NUMBER

Pennsylvania State University

University Park, Pennsylvania 16802

E-8605

\section{SPONSORING/MONITORING AGENCY NAME(S) AND ADDRESS(ES)}

10. SPONSORING/MONITORING AGENCY REPORT NUMBER

National Aeronautics and Space Administration

Washington, D.C. $20546-0001$

NASA CR-194470

\section{SUPPLEMENTARY NOTES}

Project Manager, Carl F. Lorenzo, Instrumentation and Control Technology Division, organization code 2500, NASA Lewis Research Center, (216) 433-3733.

12a. DISTRIBUTIONAVAILABILITY STATEMENT

12b. DISTRIBUTION CODE

Unclassified - Unlimited

Subject Categories 39 and 63

13. ABSTRACT (Maximum 200 words)

A major goal in the control of complex mechanical systems such as spacecraft rocket engines, advanced aircraft, and power plants is to achieve high performance with increased reliability, availability, component durability, and maintainability. The current practice of decision and control systems synthesis focuses on improving performance and diagnostic capabilities under constraints that often do not adequately represent the materials degradation. In view of the high performance requirements of the system and availability of improved materials, the lack of appropriate knowledge about the properties of these materials will lead to either less than achievable performance due to overly conservative design, or over-straining of the structure leading to unexpected failures and drastic reduction of the service life. The key idea in this report is that a significant improvement in service life could be achieved by a small reduction in the system dynamic performance. The major task is to characterize the damage generation process, and then utilize this information in a mathematical form to synthesize a control law that would meet the system requirements and simultaneously satisfy the constraints that are imposed by the material and structural properties of the critical components. The concept of damage mitigation is introduced for control of mechanical systems to achieve high performance with a prolonged life span. A model of fatigue damage dynamics is formulated in the continuous-time setting, instead of a cycle-based representation, for direct application to control systems synthesis. An optimal control policy is then formulated via nonlinear programming under specified constraints of the damage rate and accumulated damage. The results of simulation experiments for the transient upthrust of a bipropellant rocket engine are presented to demonstrate efficacy of the damage-mitigating control concept.

14. SUBJECT TERMS

Damage mitigating control; Life extending control; Damage modeling; Fatigue; Creep; Continuum damage modeling

17. SECURITY CLASSIFICATION OF REPORT Unclassified
18. SECURITY CLASSIFICATION OF THIS PAGE

Unclassified
15. NUMBER OF PAGES

89

16. PRICE CODE

A04 